UCRL-ID-131877

\title{
TOPAZ3D Validation Report
}

\author{
Eugene Kang
}

July 1, 1998

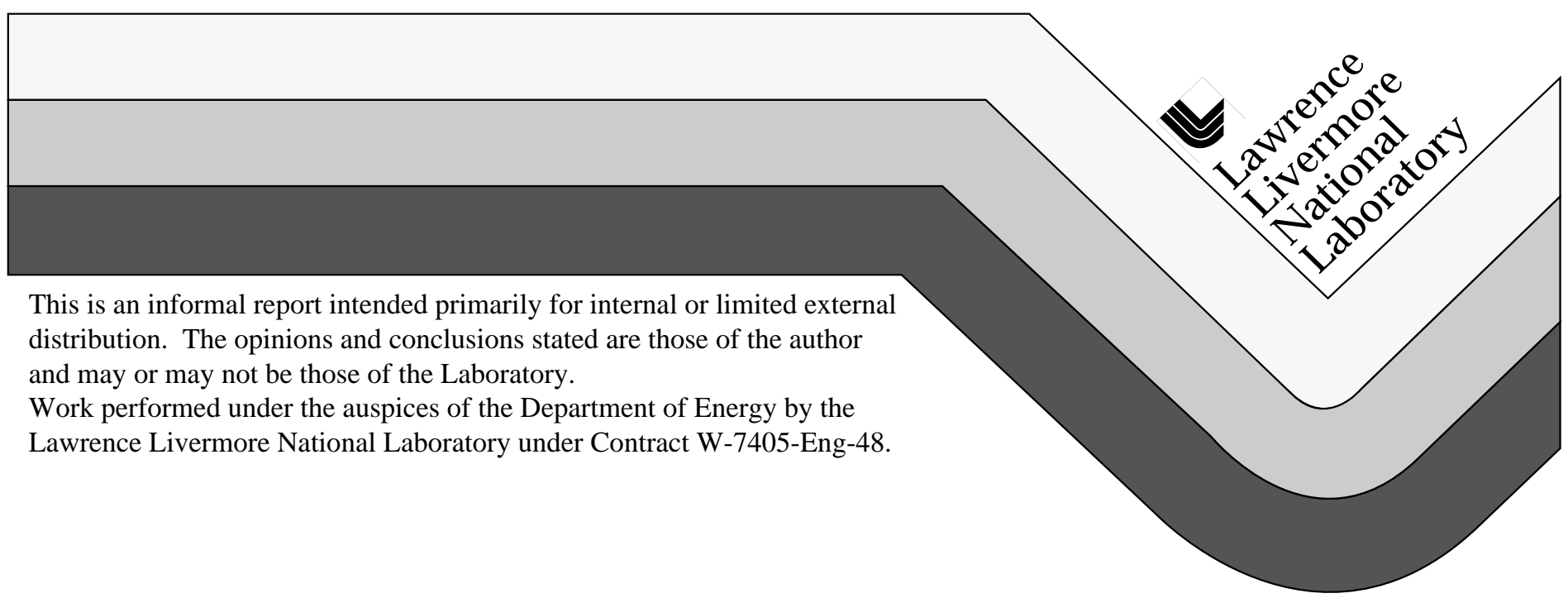




\section{DISCLAIMER}

This document was prepared as an account of work sponsored by an agency of the United States Government. Neither the United States Government nor the University of California nor any of their employees, makes any warranty, express or implied, or assumes any legal liability or responsibility for the accuracy, completeness, or usefulness of any information, apparatus, product, or process disclosed, or represents that its use would not infringe privately owned rights. Reference herein to any specific commercial product, process, or service by trade name, trademark, manufacturer, or otherwise, does not necessarily constitute or imply its endorsement, recommendation, or favoring by the United States Government or the University of California. The views and opinions of authors expressed herein do not necessarily state or reflect those of the United States Government or the University of California, and shall not be used for advertising or product endorsement purposes.

This report has been reproduced directly from the best available copy.

Available to DOE and DOE contractors from the Office of Scientific and Technical Information

P.O. Box 62, Oak Ridge, TN 37831

Prices available from (615) 576-8401, FTS 626-8401

Available to the public from the

National Technical Information Service

U.S. Department of Commerce

5285 Port Royal Rd.

Springfield, VA 22161 


\title{
TOPAZ3D Validation Report \\ July 1998
}

Eugene Kang

Lawrence Livermore National Laboratory

\begin{abstract}
This document presents thirteen verification problems for the TOPAZ3D threedimensional finite-element heat transfer code. Code verification is performed by comparing TOPAZ3D-generated solutions to analytical solutions for a variety of problems, including those involving slide lines and internal elements. This verification examines linear, one-dimensional problems of both steady and transient nature. In all thirteen comparisons conducted, TOPAZ3D performs well. The results and input files are listed so as to make the verifications easily reproducible.
\end{abstract}




\section{Table of Contents}

$1.0 \quad$ Introduction ............................................................... 3

2.0 Steady Linear Problems .................................................. 5

2.1 Temperature Boundary Condition ................................. 6

2.2 Flux Boundary Condition ......................................... 8

2.3 Convection Boundary Condition .................................... 10

$2.4 \quad$ Slide Line ..................................................... 12

2.5 Special Internal Element ......................................... 15

3.0 Transient Linear Problems ................................................ 18

3.1 Constant Temperature Boundary Condition ........................ 19

3.2 Constant Flux Boundary Condition .................................. 23

3.3 Constant Convection Boundary Condition ......................... 27

3.4 Constant Slide Line .................................................... 31

3.5 Constant Special Internal Element .................................. 35

3.6 Temporal Temperature Boundary Condition ....................... 39

3.7 Temporal Flux Boundary Condition ............................... 42

3.8 Temporal Convection Boundary Condition ........................ 45

4.0 Bibliography .......................................................... 48 


\subsection{Introduction}

This purpose of this project is to verify the accuracy of the TOPAZ3D threedimensional finite-element heat transfer code by comparing TOPAZ3D-generated solutions to analytical solutions for a number of heat transfer problems. Although TOPAZ3D is capable of performing three-dimensional analysis, only one-dimensional problems were examined for the purposes of this verification. Only linear problems are covered here, both steady and transient.

The types of problems used for comparison were chosen in order to test as many possible aspects of TOPAZ3D in a simple and efficient manner. By verifying simple onedimensional conduction with temperature boundary conditions first, the more complex

elements such as slide lines or internal elements could be verified with problems containing temperature boundary conditions. In this way the effect of these slide lines and such could be isolated. In general, the following types of problems were examined: temperature boundary condition, flux boundary condition, convection boundary condition, slide line, and internal element.

The mesh used for most of the problems consisted of a single block with a length of 1.0 in the $\mathrm{x}$-direction divided into ten equal elements. The $\mathrm{y}$ - and z-direction lengths are irrelevant, but in this case lengths of 0.1 were used for those dimensions. Some of the problems were performed with a block of length 10.0 in the x-direction. For slide line and internal element problems, two separate blocks were used, although for the slide line problems the coordinates of the two blocks may overlap so as to make the object appear as a single block. For all of the problems, the material properties (conductivity, density, heat capacity, etc.) were assigned a value of 1.0. 
Truegrid was the program used to generate the meshes and also to create some of TOPAZ3D input files. However, the input files created by Truegrid do not contain the proper formatting of control cards required by TOPAZ3D. Care needs to be exercised to make sure that the input file control cards are formatted in a manner consistent with the needs of TOPAZ3D. For example, Truegrid allows a thermal contact resistance to be defined for a slide line; however, TOPAZ3D will read that number as a thermal conductance. Many other examples of this sort of error abound, which will result in either incorrect answers or a failure of the code to run.

\subsection{Steady Linear Problems}

The problems in this section model steady, linear flow of heat. In all of these problems $\mathrm{L}=1$; note that for the slide line the total length of the slab is $2 \mathrm{~L}$ and for the internal element each block is of length $\mathrm{L}$.

The problems represented in this section are:

2.1 - Temperature Boundary Condition

2.2 - Flux Boundary Condition

\section{3 - Convection Boundary Condition}

2.4 - Slide Line

2.5 - Special Internal Element

\subsection{Temperature Boundary Condition}

Problem: $\quad$ This models the steady linear flow of heat in a slab with temperature boundary conditions specified at $\mathrm{x}=0$ and $\mathrm{x}=\mathrm{L}$.

Analytic solution: $\quad T_{x}=T_{0}+\frac{x}{L}\left(T_{L}-T_{0}\right)$

Case parameters: $\quad$ Slab length - $\quad L=1$ 


$$
\text { Boundary conditions } \begin{aligned}
-T_{0} & =0 \text { at } x=0 \\
T_{L} & =1 \text { at } x=1
\end{aligned}
$$

\begin{tabular}{|c|c|c|}
\hline$\underline{\mathrm{x} \text {-coordinate }}$ & $\underline{\mathrm{T} \text { (analytical) }}$ & $\underline{\mathrm{T}}($ \\
\hline 0.00 & 0.00 & 0.0 \\
\hline 0.10 & 0.10 & 0.1 \\
\hline 0.20 & 0.20 & 0.2 \\
\hline 0.30 & 0.30 & 0.3 \\
\hline 0.40 & 0.40 & 0.4 \\
\hline 0.50 & 0.50 & 0.5 \\
\hline 0.60 & 0.60 & 0.6 \\
\hline 0.70 & 0.70 & 0.7 \\
\hline 0.80 & 0.80 & 0.8 \\
\hline 0.90 & 0.90 & 0.9 \\
\hline 1.00 & 1.00 & 1.0 \\
\hline
\end{tabular}

Results:

Input file:

c TOPAZ3D input file for steady linear temperature boundary condition problem

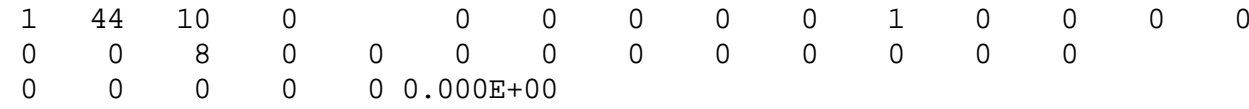

$\begin{array}{lllllllll}0.000 \mathrm{E}+00 & 0.000 \mathrm{E}+00 & 0.000 \mathrm{E}+00 & 0.000 \mathrm{E}+00 & 0.000 \mathrm{E}+00 & 0.000 \mathrm{E}+00 & 0.000 \mathrm{E}+00\end{array}$

$\begin{array}{lllllll}0 & 0 & 0 & 0 & 0 & 0.000 \mathrm{E}+00 & 0.000 \mathrm{E}+00\end{array}$

$111.000 \mathrm{E}+00 \quad 0.000 \mathrm{E}+00 \quad 0.000 \mathrm{E}+00 \quad 0 \quad 0.000 \mathrm{E}+00 \quad 0$

Material Type 1 - Isotropic

1.000E+00 1.000E+00

$1 \quad 0.0000000000000 \mathrm{E}+00 \quad 0.0000000000000 \mathrm{E}+00 \quad 0.0000000000000 \mathrm{E}+00 \quad 0.000 \mathrm{E}+00$

$2 \quad 0.0000000000000 \mathrm{E}+00 \quad 0.0000000000000 \mathrm{E}+00 \quad 1.0000000149012 \mathrm{E}-010.000 \mathrm{E}+00$

$30.0000000000000 \mathrm{E}+001.0000000149012 \mathrm{E}-010.0000000000000 \mathrm{E}+00 \quad 0.000 \mathrm{E}+00$

$4 \quad 0.0000000000000 \mathrm{E}+001.0000000149012 \mathrm{E}-011.0000000149012 \mathrm{E}-010.000 \mathrm{E}+00$

$5 \quad 1.0000000149012 \mathrm{E}-01 \quad 0.0000000000000 \mathrm{E}+00 \quad 0.0000000000000 \mathrm{E}+00 \quad 0.000 \mathrm{E}+00$

$6 \quad 1.0000000149012 \mathrm{E}-01 \quad 0.0000000000000 \mathrm{E}+00 \quad 1.0000000149012 \mathrm{E}-01 \quad 0.000 \mathrm{E}+00$

$7 \quad 1.0000000149012 \mathrm{E}-011.0000000149012 \mathrm{E}-01 \quad 0.0000000000000 \mathrm{E}+00 \quad 0.000 \mathrm{E}+00$

$8 \quad 1.0000000149012 \mathrm{E}-011.0000000149012 \mathrm{E}-011.0000000149012 \mathrm{E}-010.000 \mathrm{E}+00$

$92.0000000298023 E-010.0000000000000 E+00 \quad 0.0000000000000 E+00 \quad 0.000 E+00$

$102.0000000298023 \mathrm{E}-01 \quad 0.0000000000000 \mathrm{E}+00 \quad 1.0000000149012 \mathrm{E}-010.000 \mathrm{E}+00$

$112.0000000298023 E-01 \quad 1.0000000149012 E-010.0000000000000 E+00 \quad 0.000 E+00$

$122.0000000298023 \mathrm{E}-011.0000000149012 \mathrm{E}-011.0000000149012 \mathrm{E}-010.000 \mathrm{E}+00$

$3.0000001192093 \mathrm{E}-010.0000000000000 \mathrm{E}+00 \quad 0.0000000000000 \mathrm{E}+00 \quad 0.000 \mathrm{E}+00$

$3.0000001192093 \mathrm{E}-010.0000000000000 \mathrm{E}+00 \quad 1.0000000149012 \mathrm{E}-010.000 \mathrm{E}+00$

$3.0000001192093 \mathrm{E}-011.0000000149012 \mathrm{E}-01 \quad 0.0000000000000 \mathrm{E}+00 \quad 0.000 \mathrm{E}+00$

3.0000001192093E-01 1.0000000149012E-01 1.0000000149012E-01 0.000E+00

$4.0000000596046 \mathrm{E}-01 \quad 0.0000000000000 \mathrm{E}+00 \quad 0.0000000000000 \mathrm{E}+00 \quad 0.000 \mathrm{E}+00$

$4.0000000596046 \mathrm{E}-010.0000000000000 \mathrm{E}+00 \quad 1.0000000149012 \mathrm{E}-010.000 \mathrm{E}+00$

$4.0000000596046 \mathrm{E}-011.0000000149012 \mathrm{E}-01 \quad 0.0000000000000 \mathrm{E}+00 \quad 0.000 \mathrm{E}+00$

$4.0000000596046 \mathrm{E}-011.0000000149012 \mathrm{E}-01 \quad 1.0000000149012 \mathrm{E}-010.000 \mathrm{E}+00$

$5.0000000000000 \mathrm{E}-010.0000000000000 \mathrm{E}+00 \quad 0.0000000000000 \mathrm{E}+00 \quad 0.000 \mathrm{E}+00$

$5.0000000000000 \mathrm{E}-01 \quad 0.0000000000000 \mathrm{E}+00 \quad 1.0000000149012 \mathrm{E}-010.000 \mathrm{E}+00$

$5.0000000000000 \mathrm{E}-01 \quad 1.0000000149012 \mathrm{E}-01 \quad 0.0000000000000 \mathrm{E}+00 \quad 0.000 \mathrm{E}+00$

$5.0000000000000 \mathrm{E}-011.0000000149012 \mathrm{E}-01 \quad 1.0000000149012 \mathrm{E}-010.000 \mathrm{E}+00$

$\begin{array}{lllllll}6.0000002384186 \mathrm{E}-01 & 0.0000000000000 \mathrm{E}+00 & 0.0000000000000 \mathrm{E}+00 & 0.000 \mathrm{E}+00\end{array}$

$6.0000002384186 \mathrm{E}-010.0000000000000 \mathrm{E}+00 \quad 1.0000000149012 \mathrm{E}-010.000 \mathrm{E}+00$

$6.0000002384186 \mathrm{E}-01 \quad 1.0000000149012 \mathrm{E}-01 \quad 0.0000000000000 \mathrm{E}+00 \quad 0.000 \mathrm{E}+00$

$6.0000002384186 \mathrm{E}-011.0000000149012 \mathrm{E}-01 \quad 1.0000000149012 \mathrm{E}-010.000 \mathrm{E}+00$

$7.0000004768372 \mathrm{E}-01 \quad 0.0000000000000 \mathrm{E}+00 \quad 0.0000000000000 \mathrm{E}+00 \quad 0.000 \mathrm{E}+00$

$7.0000004768372 \mathrm{E}-01 \quad 0.0000000000000 \mathrm{E}+00 \quad 1.0000000149012 \mathrm{E}-010.000 \mathrm{E}+00$

$7.0000004768372 \mathrm{E}-01 \quad 1.0000000149012 \mathrm{E}-01 \quad 0.0000000000000 \mathrm{E}+00 \quad 0.000 \mathrm{E}+00$ 


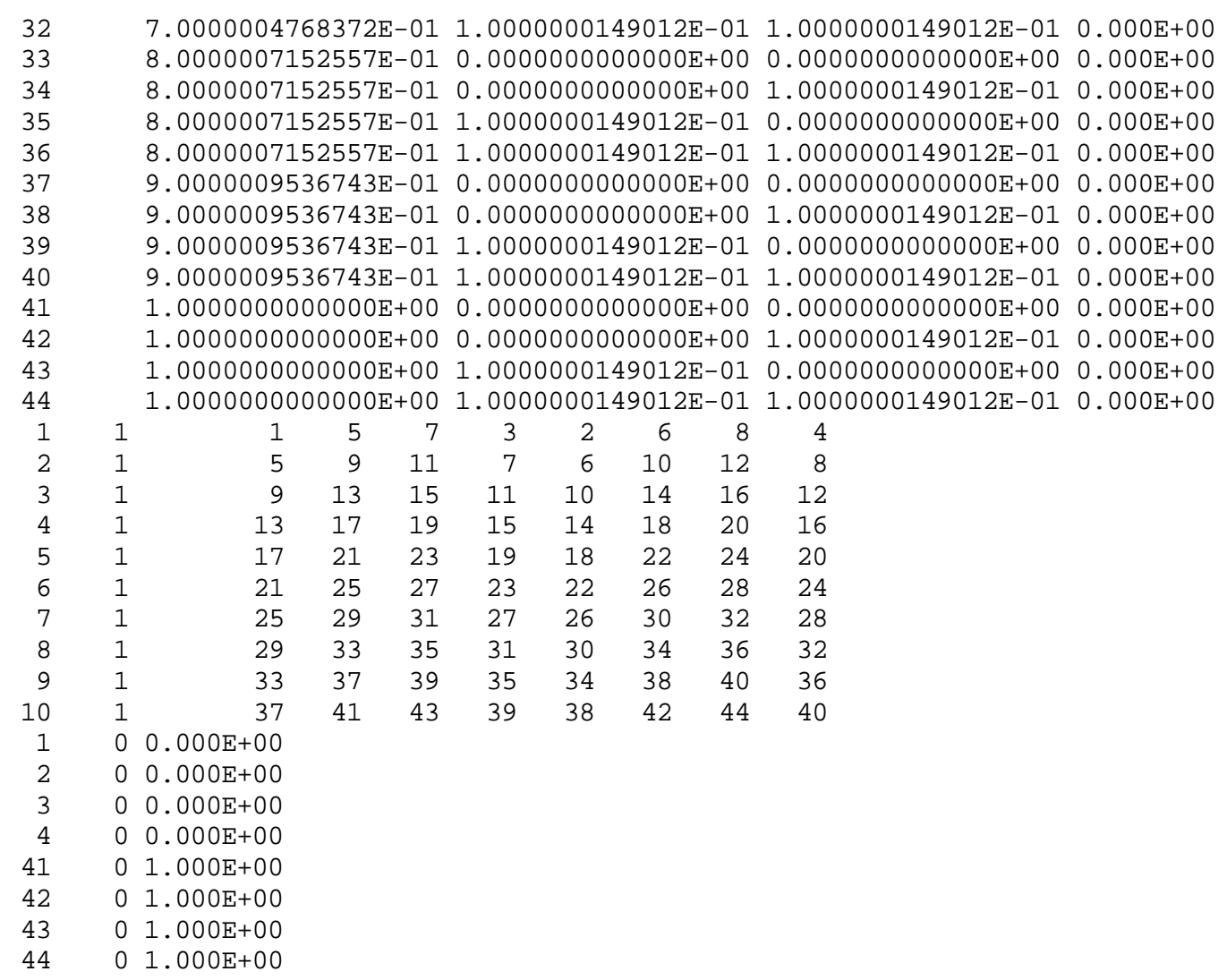

\section{$2.2 \quad$ Flux Boundary Condition}

Problem: $\quad$ This models the steady linear flow of heat in a slab with a temperature boundary condition specified at $\mathrm{x}=0$ and a uniform flux boundary condition specified at $\mathrm{x}=\mathrm{L}$.

Analytic solution: $\quad T_{x}=T_{0}+q^{\prime \prime} x$

Case parameters: $\quad$ Slab length - $\quad L=1$

Boundary conditions $-T_{0}=0$ at $x=0$

$q^{\prime \prime}=1$ at $x=1$

Results:

$\underline{\mathrm{x} \text {-coordinate }} \underline{\mathrm{T} \text { (analytical) }} \underline{\mathrm{T} \text { (TOPAZ3D) }}$

$\begin{array}{lll}0.00 & 0.00 & 0.00 \\ 0.10 & 0.10 & 0.10 \\ 0.20 & 0.20 & 0.20 \\ 0.30 & 0.30 & 0.30 \\ 0.40 & 0.40 & 0.40 \\ 0.50 & 0.50 & 0.50 \\ 0.60 & 0.60 & 0.60 \\ 0.70 & 0.70 & 0.70\end{array}$




$\begin{array}{lll}0.80 & 0.80 & 0.80 \\ 0.90 & 0.90 & 0.90 \\ 1.00 & 1.00 & 1.00\end{array}$

\section{Input file:}

C TOPAZ3D input file for steady linear flux boundary condition problem

$\begin{array}{lllllllllllllll}1 & 44 & 10 & 0 & 0 & 0 & 0 & 0 & 0 & 1 & 0 & 0 & 0 & 0\end{array}$

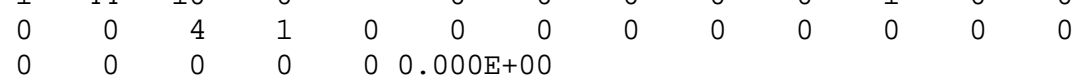

$\begin{array}{llllllll}0.000 \mathrm{E}+00 & 0.000 \mathrm{E}+00 & 0.000 \mathrm{E}+00 & 0.000 \mathrm{E}+00 & 0.000 \mathrm{E}+00 & 0.000 \mathrm{E}+00 & 0.000 \mathrm{E}+00\end{array}$

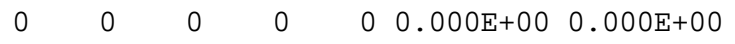

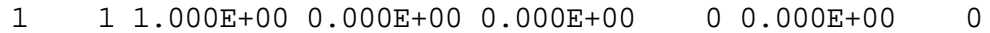

Material Type 1 - Isotropic

$1.000 \mathrm{E}+001.000 \mathrm{E}+00$

$1 \quad 0.0000000000000 \mathrm{E}+00 \quad 0.0000000000000 \mathrm{E}+00 \quad 0.0000000000000 \mathrm{E}+00 \quad 0.000 \mathrm{E}+00$

$2 \quad 0.0000000000000 \mathrm{E}+00 \quad 0.0000000000000 \mathrm{E}+00 \quad 1.0000000149012 \mathrm{E}-01 \quad 0.000 \mathrm{E}+00$

$3 \quad 0.0000000000000 \mathrm{E}+00 \quad 1.0000000149012 \mathrm{E}-01 \quad 0.0000000000000 \mathrm{E}+00 \quad 0.000 \mathrm{E}+00$

$4 \quad 0.0000000000000 \mathrm{E}+001.0000000149012 \mathrm{E}-01 \quad 1.0000000149012 \mathrm{E}-010.000 \mathrm{E}+00$

$5 \quad 1.0000000149012 \mathrm{E}-01 \quad 0.0000000000000 \mathrm{E}+00 \quad 0.0000000000000 \mathrm{E}+00 \quad 0.000 \mathrm{E}+00$

$6 \quad 1.0000000149012 \mathrm{E}-01 \quad 0.0000000000000 \mathrm{E}+00 \quad 1.0000000149012 \mathrm{E}-010.000 \mathrm{E}+00$

$7 \quad 1.0000000149012 \mathrm{E}-01 \quad 1.0000000149012 \mathrm{E}-01 \quad 0.0000000000000 \mathrm{E}+00 \quad 0.000 \mathrm{E}+00$

$8 \quad 1.0000000149012 \mathrm{E}-01 \quad 1.0000000149012 \mathrm{E}-01 \quad 1.0000000149012 \mathrm{E}-010.000 \mathrm{E}+00$

$92.0000000298023 \mathrm{E}-01 \quad 0.0000000000000 \mathrm{E}+00 \quad 0.0000000000000 \mathrm{E}+00 \quad 0.000 \mathrm{E}+00$

$102.0000000298023 \mathrm{E}-01 \quad 0.0000000000000 \mathrm{E}+00 \quad 1.0000000149012 \mathrm{E}-01 \quad 0.000 \mathrm{E}+00$

$112.0000000298023 \mathrm{E}-01 \quad 1.0000000149012 \mathrm{E}-01 \quad 0.0000000000000 \mathrm{E}+00 \quad 0.000 \mathrm{E}+00$

$122.0000000298023 \mathrm{E}-01 \quad 1.0000000149012 \mathrm{E}-01 \quad 1.0000000149012 \mathrm{E}-010.000 \mathrm{E}+00$

$133.0000001192093 \mathrm{E}-01 \quad 0.0000000000000 \mathrm{E}+00 \quad 0.0000000000000 \mathrm{E}+00 \quad 0.000 \mathrm{E}+00$

$143.0000001192093 \mathrm{E}-01 \quad 0.0000000000000 \mathrm{E}+001.0000000149012 \mathrm{E}-010.000 \mathrm{E}+00$

$153.0000001192093 \mathrm{E}-01 \quad 1.0000000149012 \mathrm{E}-01 \quad 0.0000000000000 \mathrm{E}+00 \quad 0.000 \mathrm{E}+00$

$163.0000001192093 \mathrm{E}-01 \quad 1.0000000149012 \mathrm{E}-01 \quad 1.0000000149012 \mathrm{E}-010.000 \mathrm{E}+00$

$174.0000000596046 \mathrm{E}-01 \quad 0.0000000000000 \mathrm{E}+00 \quad 0.0000000000000 \mathrm{E}+00 \quad 0.000 \mathrm{E}+00$

$184.0000000596046 \mathrm{E}-01 \quad 0.0000000000000 \mathrm{E}+00 \quad 1.0000000149012 \mathrm{E}-01 \quad 0.000 \mathrm{E}+00$

$194.0000000596046 \mathrm{E}-01 \quad 1.0000000149012 \mathrm{E}-01 \quad 0.0000000000000 \mathrm{E}+00 \quad 0.000 \mathrm{E}+00$

$204.0000000596046 \mathrm{E}-01 \quad 1.0000000149012 \mathrm{E}-01 \quad 1.0000000149012 \mathrm{E}-01 \quad 0.000 \mathrm{E}+00$

$215.0000000000000 \mathrm{E}-01 \quad 0.0000000000000 \mathrm{E}+00 \quad 0.0000000000000 \mathrm{E}+00 \quad 0.000 \mathrm{E}+00$

$225.0000000000000 \mathrm{E}-01 \quad 0.0000000000000 \mathrm{E}+001.0000000149012 \mathrm{E}-010.000 \mathrm{E}+00$

$235.0000000000000 \mathrm{E}-01 \quad 1.0000000149012 \mathrm{E}-01 \quad 0.0000000000000 \mathrm{E}+00 \quad 0.000 \mathrm{E}+00$

$245.0000000000000 \mathrm{E}-01 \quad 1.0000000149012 \mathrm{E}-01 \quad 1.0000000149012 \mathrm{E}-010.000 \mathrm{E}+00$

$256.0000002384186 \mathrm{E}-01 \quad 0.0000000000000 \mathrm{E}+00 \quad 0.0000000000000 \mathrm{E}+00 \quad 0.000 \mathrm{E}+00$

$266.0000002384186 \mathrm{E}-01 \quad 0.0000000000000 \mathrm{E}+00 \quad 1.0000000149012 \mathrm{E}-010.000 \mathrm{E}+00$

$276.0000002384186 \mathrm{E}-01 \quad 1.0000000149012 \mathrm{E}-01 \quad 0.0000000000000 \mathrm{E}+00 \quad 0.000 \mathrm{E}+00$

$286.0000002384186 \mathrm{E}-01 \quad 1.0000000149012 \mathrm{E}-01 \quad 1.0000000149012 \mathrm{E}-010.000 \mathrm{E}+00$

$297.0000004768372 \mathrm{E}-01 \quad 0.0000000000000 \mathrm{E}+00 \quad 0.0000000000000 \mathrm{E}+00 \quad 0.000 \mathrm{E}+00$

$307.0000004768372 \mathrm{E}-01 \quad 0.0000000000000 \mathrm{E}+00 \quad 1.0000000149012 \mathrm{E}-01 \quad 0.000 \mathrm{E}+00$

$317.0000004768372 \mathrm{E}-01 \quad 1.0000000149012 \mathrm{E}-01 \quad 0.0000000000000 \mathrm{E}+00 \quad 0.000 \mathrm{E}+00$

$327.0000004768372 \mathrm{E}-01 \quad 1.0000000149012 \mathrm{E}-01 \quad 1.0000000149012 \mathrm{E}-010.000 \mathrm{E}+00$

$338.0000007152557 \mathrm{E}-01 \quad 0.0000000000000 \mathrm{E}+00 \quad 0.0000000000000 \mathrm{E}+00 \quad 0.000 \mathrm{E}+00$

$348.0000007152557 \mathrm{E}-01 \quad 0.0000000000000 \mathrm{E}+00 \quad 1.0000000149012 \mathrm{E}-01 \quad 0.000 \mathrm{E}+00$

$358.0000007152557 \mathrm{E}-01 \quad 1.0000000149012 \mathrm{E}-01 \quad 0.0000000000000 \mathrm{E}+00 \quad 0.000 \mathrm{E}+00$

$36 \quad 8.0000007152557 \mathrm{E}-01 \quad 1.0000000149012 \mathrm{E}-01 \quad 1.0000000149012 \mathrm{E}-010.000 \mathrm{E}+00$

$379.0000009536743 \mathrm{E}-01 \quad 0.0000000000000 \mathrm{E}+00 \quad 0.0000000000000 \mathrm{E}+00 \quad 0.000 \mathrm{E}+00$

$389.0000009536743 \mathrm{E}-01 \quad 0.0000000000000 \mathrm{E}+00 \quad 1.0000000149012 \mathrm{E}-01 \quad 0.000 \mathrm{E}+00$

$399.0000009536743 \mathrm{E}-01 \quad 1.0000000149012 \mathrm{E}-01 \quad 0.0000000000000 \mathrm{E}+00 \quad 0.000 \mathrm{E}+00$

$409.0000009536743 \mathrm{E}-01 \quad 1.0000000149012 \mathrm{E}-01 \quad 1.0000000149012 \mathrm{E}-01 \quad 0.000 \mathrm{E}+00$

$41 \quad 1.0000000000000 \mathrm{E}+00 \quad 0.0000000000000 \mathrm{E}+00 \quad 0.0000000000000 \mathrm{E}+00 \quad 0.000 \mathrm{E}+00$

$42 \quad 1.0000000000000 \mathrm{E}+00 \quad 0.0000000000000 \mathrm{E}+00 \quad 1.0000000149012 \mathrm{E}-01 \quad 0.000 \mathrm{E}+00$

$43 \quad 1.0000000000000 \mathrm{E}+00 \quad 1.0000000149012 \mathrm{E}-01 \quad 0.0000000000000 \mathrm{E}+00 \quad 0.000 \mathrm{E}+00$

$441.0000000000000 \mathrm{E}+001.0000000149012 \mathrm{E}-01 \quad 1.0000000149012 \mathrm{E}-010.000 \mathrm{E}+00$

$\begin{array}{rrrrrrrrrr}1 & 1 & 1 & 5 & 7 & 3 & 2 & 6 & 8 & 4 \\ 2 & 1 & 5 & 9 & 11 & 7 & 6 & 10 & 12 & 8 \\ 3 & 1 & 9 & 13 & 15 & 11 & 10 & 14 & 16 & 12 \\ 4 & 1 & 13 & 17 & 19 & 15 & 14 & 18 & 20 & 16\end{array}$




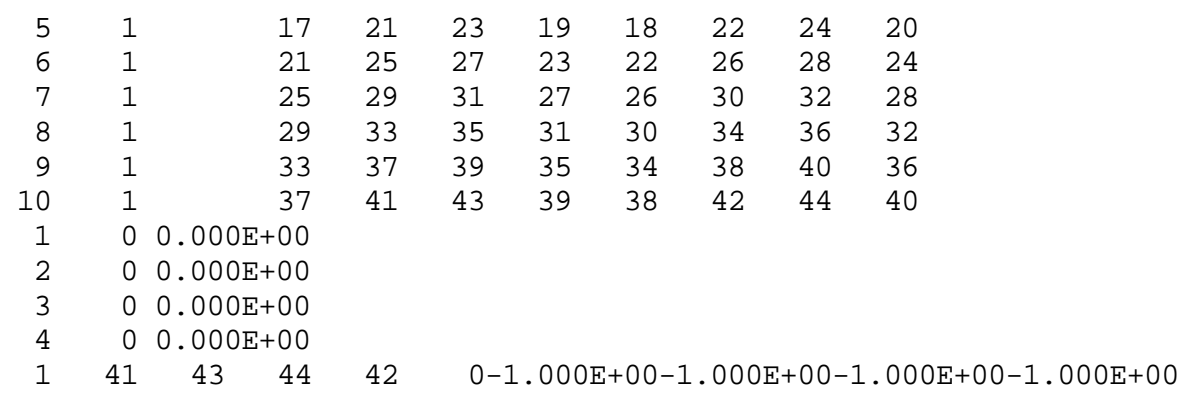

\subsection{Convection Boundary Condition}

Problem: $\quad$ This case models the steady linear flow of heat in a slab with a temperature boundary condition specified at $\mathrm{x}=0$ and a convection boundary condition specified at $\mathrm{x}=\mathrm{L}$.

Analytic solution: $\quad T=T_{0}+\frac{x}{k}\left(\frac{T_{\infty}-T_{0}}{\frac{L}{k}+\frac{1}{h}}\right)$

Case parameters: $\quad$ Slab length - $\quad L=1$

Boundary conditions $-T_{0}=1$ at $x=0$

$$
q=h\left(T_{\infty}-T_{L}\right) \text { at } x=\mathrm{L} ; h=1, T_{\infty}=0
$$

Results:

$\underline{\mathrm{x} \text {-coordinate }} \underline{\mathrm{T} \text { (analytical) }} \underline{\mathrm{T} \text { (TOPAZ3D) }}$

$\begin{array}{lll}0.00 & 1.00 & 1.00 \\ 0.10 & 0.95 & 0.95 \\ 0.20 & 0.90 & 0.90 \\ 0.30 & 0.85 & 0.85 \\ 0.40 & 0.80 & 0.80 \\ 0.50 & 0.75 & 0.75 \\ 0.60 & 0.70 & 0.70 \\ 0.70 & 0.65 & 0.65 \\ 0.80 & 0.60 & 0.60 \\ 0.90 & 0.55 & 0.55 \\ 1.00 & 0.50 & 0.50\end{array}$

Input file:

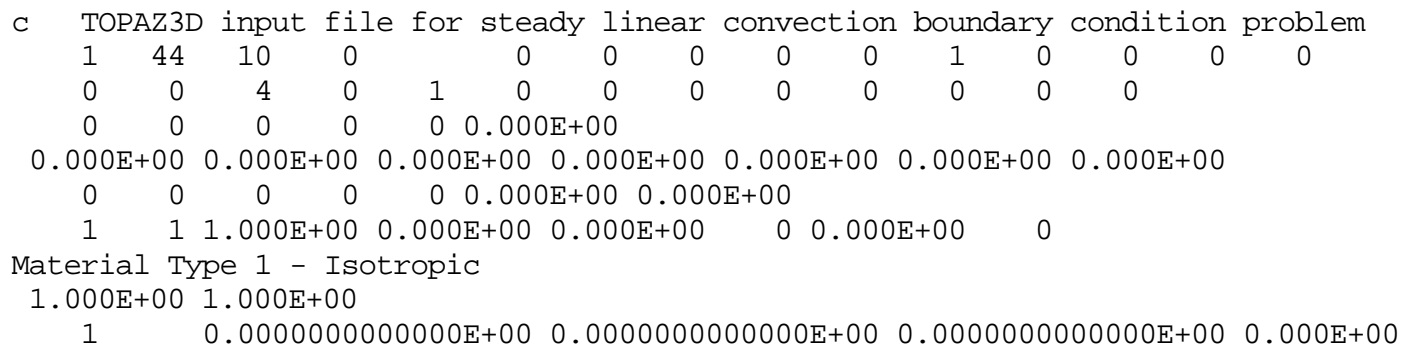


$2 \quad 0.0000000000000 \mathrm{E}+00 \quad 0.0000000000000 \mathrm{E}+00 \quad 1.0000000149012 \mathrm{E}-010.000 \mathrm{E}+00$ $3 \quad 0.0000000000000 \mathrm{E}+00 \quad 1.0000000149012 \mathrm{E}-01 \quad 0.0000000000000 \mathrm{E}+00 \quad 0.000 \mathrm{E}+00$ $4 \quad 0.0000000000000 \mathrm{E}+001.0000000149012 \mathrm{E}-01 \quad 1.0000000149012 \mathrm{E}-01 \quad 0.000 \mathrm{E}+00$ $5 \quad 1.0000000149012 \mathrm{E}-01 \quad 0.0000000000000 \mathrm{E}+00 \quad 0.0000000000000 \mathrm{E}+00 \quad 0.000 \mathrm{E}+00$ $6 \quad 1.0000000149012 \mathrm{E}-01 \quad 0.0000000000000 \mathrm{E}+00 \quad 1.0000000149012 \mathrm{E}-01 \quad 0.000 \mathrm{E}+00$ $7 \quad 1.0000000149012 \mathrm{E}-01 \quad 1.0000000149012 \mathrm{E}-01 \quad 0.0000000000000 \mathrm{E}+00 \quad 0.000 \mathrm{E}+00$

$1.0000000149012 \mathrm{E}-01 \quad 1.0000000149012 \mathrm{E}-01 \quad 1.0000000149012 \mathrm{E}-01 \quad 0.000 \mathrm{E}+00$ $2.0000000298023 \mathrm{E}-01 \quad 0.0000000000000 \mathrm{E}+00 \quad 0.0000000000000 \mathrm{E}+00 \quad 0.000 \mathrm{E}+00$ $2.0000000298023 \mathrm{E}-01 \quad 0.0000000000000 \mathrm{E}+00 \quad 1.0000000149012 \mathrm{E}-01 \quad 0.000 \mathrm{E}+00$ $2.0000000298023 \mathrm{E}-01 \quad 1.0000000149012 \mathrm{E}-01 \quad 0.0000000000000 \mathrm{E}+00 \quad 0.000 \mathrm{E}+00$ $2.0000000298023 \mathrm{E}-01 \quad 1.0000000149012 \mathrm{E}-01 \quad 1.0000000149012 \mathrm{E}-01 \quad 0.000 \mathrm{E}+00$ $3.0000001192093 \mathrm{E}-01 \quad 0.0000000000000 \mathrm{E}+00 \quad 0.0000000000000 \mathrm{E}+00 \quad 0.000 \mathrm{E}+00$ 3.0000001192093E-01 $0.0000000000000 \mathrm{E}+00 \quad 1.0000000149012 \mathrm{E}-01 \quad 0.000 \mathrm{E}+00$ 3.0000001192093E-01 1.0000000149012E-01 $0.0000000000000 \mathrm{E}+00 \quad 0.000 \mathrm{E}+00$ $3.0000001192093 \mathrm{E}-01 \quad 1.0000000149012 \mathrm{E}-01 \quad 1.0000000149012 \mathrm{E}-01 \quad 0.000 \mathrm{E}+00$ $\begin{array}{lllll}4.0000000596046 \mathrm{E}-01 & 0.0000000000000 \mathrm{E}+00 & 0.0000000000000 \mathrm{E}+00 & 0.000 \mathrm{E}+00\end{array}$ $\begin{array}{llllll}4.0000000596046 \mathrm{E}-01 & 0.0000000000000 \mathrm{E}+00 & 1.0000000149012 \mathrm{E}-01 & 0.000 \mathrm{E}+00\end{array}$ $4.0000000596046 \mathrm{E}-01 \quad 1.0000000149012 \mathrm{E}-010.0000000000000 \mathrm{E}+00 \quad 0.000 \mathrm{E}+00$ $\begin{array}{lllll}4.0000000596046 \mathrm{E}-01 & 1.0000000149012 \mathrm{E}-01 & 1.0000000149012 \mathrm{E}-01 & 0.000 \mathrm{E}+00\end{array}$ $5.0000000000000 \mathrm{E}-01 \quad 0.0000000000000 \mathrm{E}+00 \quad 0.0000000000000 \mathrm{E}+00 \quad 0.000 \mathrm{E}+00$ $5.0000000000000 \mathrm{E}-01 \quad 0.0000000000000 \mathrm{E}+00 \quad 1.0000000149012 \mathrm{E}-01 \quad 0.000 \mathrm{E}+00$ $5.0000000000000 \mathrm{E}-01 \quad 1.0000000149012 \mathrm{E}-01 \quad 0.0000000000000 \mathrm{E}+00 \quad 0.000 \mathrm{E}+00$ $5.0000000000000 \mathrm{E}-01 \quad 1.0000000149012 \mathrm{E}-01 \quad 1.0000000149012 \mathrm{E}-01 \quad 0.000 \mathrm{E}+00$ $\begin{array}{llllll}6.0000002384186 \mathrm{E}-01 & 0.0000000000000 \mathrm{E}+00 & 0.0000000000000 \mathrm{E}+00 & 0.000 \mathrm{E}+00\end{array}$ $6.0000002384186 \mathrm{E}-01 \quad 0.0000000000000 \mathrm{E}+00 \quad 1.0000000149012 \mathrm{E}-01 \quad 0.000 \mathrm{E}+00$ $6.0000002384186 \mathrm{E}-01 \quad 1.0000000149012 \mathrm{E}-01 \quad 0.0000000000000 \mathrm{E}+00 \quad 0.000 \mathrm{E}+00$ $6.0000002384186 \mathrm{E}-01 \quad 1.0000000149012 \mathrm{E}-01 \quad 1.0000000149012 \mathrm{E}-01 \quad 0.000 \mathrm{E}+00$ $\begin{array}{lllll}7.0000004768372 \mathrm{E}-01 & 0.0000000000000 \mathrm{E}+00 & 0.0000000000000 \mathrm{E}+00 & 0.000 \mathrm{E}+00\end{array}$ $\begin{array}{lllll}7.0000004768372 \mathrm{E}-01 & 0.0000000000000 \mathrm{E}+00 & 1.0000000149012 \mathrm{E}-01 & 0.000 \mathrm{E}+00\end{array}$ $\begin{array}{lllll}7.0000004768372 \mathrm{E}-01 & 1.0000000149012 \mathrm{E}-01 & 0.0000000000000 \mathrm{E}+00 & 0.000 \mathrm{E}+00\end{array}$ $7.0000004768372 \mathrm{E}-01 \quad 1.0000000149012 \mathrm{E}-01 \quad 1.0000000149012 \mathrm{E}-01 \quad 0.000 \mathrm{E}+00$ $8.0000007152557 \mathrm{E}-01 \quad 0.0000000000000 \mathrm{E}+00 \quad 0.0000000000000 \mathrm{E}+00 \quad 0.000 \mathrm{E}+00$ $8.0000007152557 \mathrm{E}-01 \quad 0.0000000000000 \mathrm{E}+00 \quad 1.0000000149012 \mathrm{E}-01 \quad 0.000 \mathrm{E}+00$ $8.0000007152557 \mathrm{E}-01 \quad 1.0000000149012 \mathrm{E}-01 \quad 0.0000000000000 \mathrm{E}+00 \quad 0.000 \mathrm{E}+00$ $8.0000007152557 \mathrm{E}-01 \quad 1.0000000149012 \mathrm{E}-01 \quad 1.0000000149012 \mathrm{E}-01 \quad 0.000 \mathrm{E}+00$ $9.0000009536743 \mathrm{E}-01 \quad 0.0000000000000 \mathrm{E}+00 \quad 0.0000000000000 \mathrm{E}+00 \quad 0.000 \mathrm{E}+00$ $9.0000009536743 \mathrm{E}-01 \quad 0.0000000000000 \mathrm{E}+00 \quad 1.0000000149012 \mathrm{E}-01 \quad 0.000 \mathrm{E}+00$ $9.0000009536743 \mathrm{E}-01 \quad 1.0000000149012 \mathrm{E}-01 \quad 0.0000000000000 \mathrm{E}+00 \quad 0.000 \mathrm{E}+00$ $9.0000009536743 \mathrm{E}-01 \quad 1.0000000149012 \mathrm{E}-01 \quad 1.0000000149012 \mathrm{E}-01 \quad 0.000 \mathrm{E}+00$ $1.0000000000000 \mathrm{E}+00 \quad 0.0000000000000 \mathrm{E}+00 \quad 0.0000000000000 \mathrm{E}+00 \quad 0.000 \mathrm{E}+00$ $1.0000000000000 \mathrm{E}+00 \quad 0.0000000000000 \mathrm{E}+00 \quad 1.0000000149012 \mathrm{E}-01 \quad 0.000 \mathrm{E}+00$ $1.0000000000000 \mathrm{E}+00 \quad 1.0000000149012 \mathrm{E}-01 \quad 0.0000000000000 \mathrm{E}+00 \quad 0.000 \mathrm{E}+00$ $1.0000000000000 \mathrm{E}+001.0000000149012 \mathrm{E}-01 \quad 1.0000000149012 \mathrm{E}-01 \quad 0.000 \mathrm{E}+00$

\begin{tabular}{|c|c|c|c|c|c|c|c|c|}
\hline 1 & 1 & 5 & 7 & 3 & 2 & 6 & 8 & 4 \\
\hline 1 & 5 & 9 & 11 & 7 & 6 & 10 & 12 & 8 \\
\hline 1 & 9 & 13 & 15 & 11 & 10 & 14 & 16 & 12 \\
\hline 1 & 13 & 17 & 19 & 15 & 14 & 18 & 20 & 16 \\
\hline 1 & 17 & 21 & 23 & 19 & 18 & 22 & 24 & 20 \\
\hline 1 & 21 & 25 & 27 & 23 & 22 & 26 & 28 & 24 \\
\hline 1 & 25 & 29 & 31 & 27 & 26 & 30 & 32 & 28 \\
\hline 1 & 29 & 33 & 35 & 31 & 30 & 34 & 36 & 32 \\
\hline 1 & 33 & 37 & 39 & 35 & 34 & 38 & 40 & 36 \\
\hline 1 & 37 & 41 & 43 & 39 & 38 & 42 & 44 & 40 \\
\hline \multicolumn{9}{|c|}{$01.000 \mathrm{E}+00$} \\
\hline 0 & \multicolumn{8}{|c|}{$1.000 \mathrm{E}+00$} \\
\hline 0 & \multicolumn{8}{|c|}{$1.000 \mathrm{E}+00$} \\
\hline 0 & \multicolumn{8}{|c|}{$1.000 \mathrm{E}+00$} \\
\hline 41 & $43 \quad 44$ & 42 & 0 & .000 & +00 & 0 & .000 & +00 \\
\hline
\end{tabular}

\section{4 \\ Problem:}

\section{Slide Line}

This models the steady linear flow of heat in a slab with temperature boundary conditions specified at $\mathrm{x}=0$ and $\mathrm{x}=2 \mathrm{~L}$ and a slide line interface with a thermal conductivity of $\mathrm{H}$ defined at $\mathrm{x}=\mathrm{L}$. Note 
that the left side of interface is defined as surface $A$ and the right as surface B.

Analytic solution: $\quad q^{\prime \prime}=H\left(T_{s 1}-T_{s 2}\right)$

Combined with the conduction equation, this yields

$$
T_{B}=\frac{T_{L}\left(\frac{k}{L}+H\right)+H T_{0}}{\frac{k}{L}+2 H}, T_{A}=T_{L}+T_{0}-T_{B}
$$

Case parameters: $\quad$ Slab length - length $=2 L=2$

Boundary conditions $-T_{0}=1$ at $x=0$

$T_{L}=2$ at $x=2$

$H=0.5$

$\underline{\text { Results: }} \quad \underline{\text { Surface }} \quad \underline{T \text { (analytical) }} \underline{T \text { (TOPAZ3D) }}$

$\begin{array}{lll}\text { A } & 1.25 & 1.25 \\ \text { B } & 1.75 & 1.75\end{array}$

Note: The rest of the object behaves under the laws governing onedimensional linear conduction, and the temperatures at various $\mathrm{x}-$ coordinates can be determined and verified easily.

\section{Input file:}

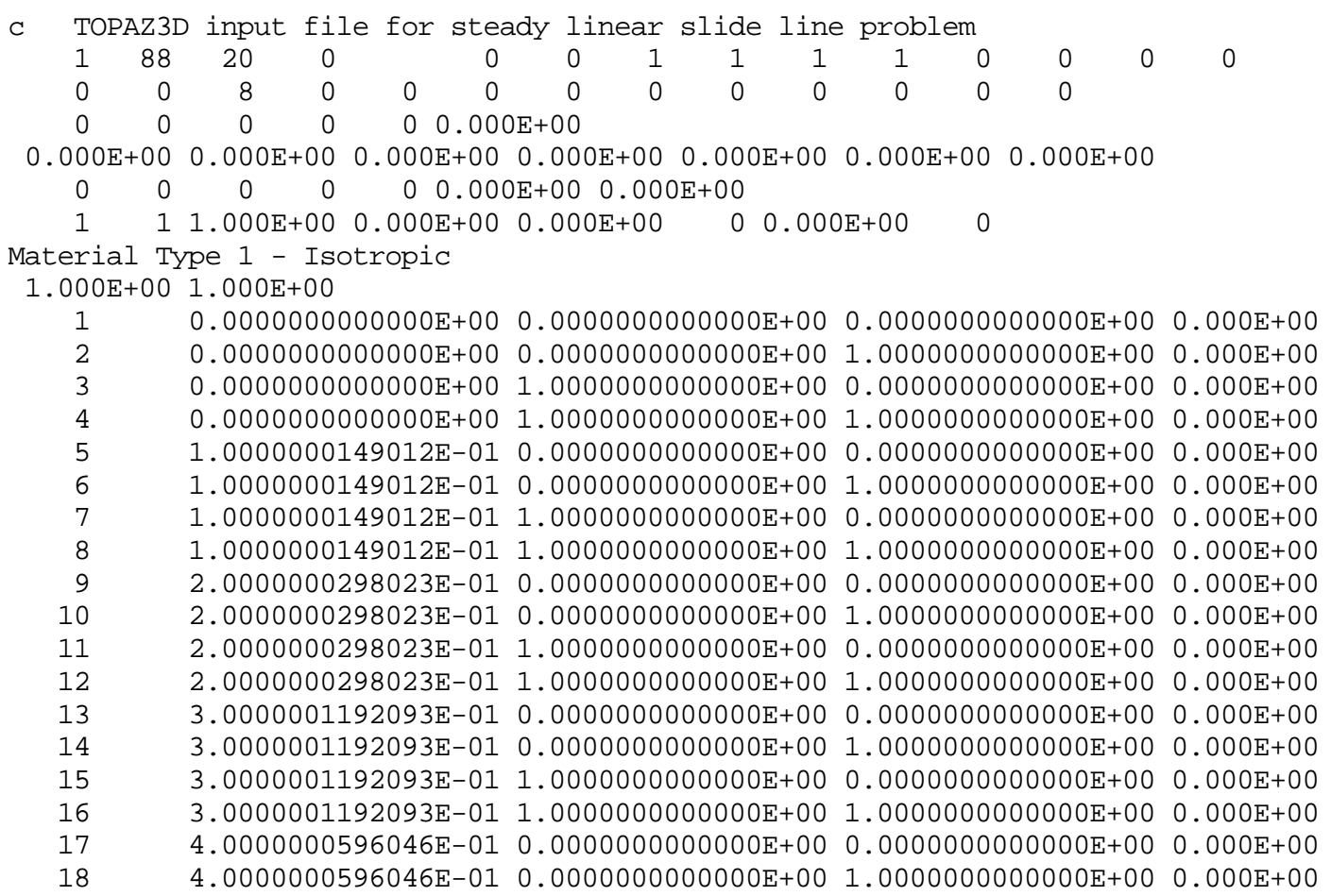


$4.0000000596046 \mathrm{E}-01 \quad 1.0000000000000 \mathrm{E}+00 \quad 0.0000000000000 \mathrm{E}+00 \quad 0.000 \mathrm{E}+00$ $4.0000000596046 \mathrm{E}-01 \quad 1.0000000000000 \mathrm{E}+00 \quad 1.0000000000000 \mathrm{E}+00 \quad 0.000 \mathrm{E}+00$ $5.0000000000000 \mathrm{E}-01 \quad 0.0000000000000 \mathrm{E}+00 \quad 0.0000000000000 \mathrm{E}+00 \quad 0.000 \mathrm{E}+00$ $5.0000000000000 \mathrm{E}-01 \quad 0.0000000000000 \mathrm{E}+00 \quad 1.0000000000000 \mathrm{E}+00 \quad 0.000 \mathrm{E}+00$ $5.0000000000000 \mathrm{E}-01 \quad 1.0000000000000 \mathrm{E}+00 \quad 0.0000000000000 \mathrm{E}+00 \quad 0.000 \mathrm{E}+00$ $5.0000000000000 \mathrm{E}-01 \quad 1.0000000000000 \mathrm{E}+00 \quad 1.0000000000000 \mathrm{E}+00 \quad 0.000 \mathrm{E}+00$ $\begin{array}{llllll}6.0000002384186 \mathrm{E}-01 & 0.0000000000000 \mathrm{E}+00 & 0.0000000000000 \mathrm{E}+00 & 0.000 \mathrm{E}+00\end{array}$ $6.0000002384186 \mathrm{E}-01 \quad 0.0000000000000 \mathrm{E}+00 \quad 1.0000000000000 \mathrm{E}+00 \quad 0.000 \mathrm{E}+00$ $\begin{array}{lllll}6.0000002384186 \mathrm{E}-01 & 1.0000000000000 \mathrm{E}+00 & 0.0000000000000 \mathrm{E}+00 & 0.000 \mathrm{E}+00\end{array}$ $6.0000002384186 \mathrm{E}-01 \quad 1.0000000000000 \mathrm{E}+00 \quad 1.0000000000000 \mathrm{E}+00 \quad 0.000 \mathrm{E}+00$ $7.0000004768372 \mathrm{E}-01 \quad 0.0000000000000 \mathrm{E}+00 \quad 0.0000000000000 \mathrm{E}+00 \quad 0.000 \mathrm{E}+00$ $7.0000004768372 \mathrm{E}-01 \quad 0.0000000000000 \mathrm{E}+00 \quad 1.0000000000000 \mathrm{E}+00 \quad 0.000 \mathrm{E}+00$ $7.0000004768372 \mathrm{E}-01 \quad 1.0000000000000 \mathrm{E}+00 \quad 0.0000000000000 \mathrm{E}+00 \quad 0.000 \mathrm{E}+00$ $7.0000004768372 \mathrm{E}-01 \quad 1.0000000000000 \mathrm{E}+00 \quad 1.0000000000000 \mathrm{E}+00 \quad 0.000 \mathrm{E}+00$ $8.0000007152557 \mathrm{E}-01 \quad 0.0000000000000 \mathrm{E}+00 \quad 0.0000000000000 \mathrm{E}+00 \quad 0.000 \mathrm{E}+00$ $8.0000007152557 \mathrm{E}-01 \quad 0.0000000000000 \mathrm{E}+00 \quad 1.0000000000000 \mathrm{E}+00 \quad 0.000 \mathrm{E}+00$ $8.0000007152557 \mathrm{E}-01 \quad 1.0000000000000 \mathrm{E}+00 \quad 0.0000000000000 \mathrm{E}+00 \quad 0.000 \mathrm{E}+00$ $8.0000007152557 \mathrm{E}-011.0000000000000 \mathrm{E}+00 \quad 1.0000000000000 \mathrm{E}+00 \quad 0.000 \mathrm{E}+00$ $\begin{array}{lllll}9.0000009536743 \mathrm{E}-01 & 0.0000000000000 \mathrm{E}+00 & 0.0000000000000 \mathrm{E}+00 & 0.000 \mathrm{E}+00\end{array}$ $9.0000009536743 \mathrm{E}-01 \quad 0.0000000000000 \mathrm{E}+00 \quad 1.0000000000000 \mathrm{E}+00 \quad 0.000 \mathrm{E}+00$ $9.0000009536743 \mathrm{E}-01 \quad 1.0000000000000 \mathrm{E}+00 \quad 0.0000000000000 \mathrm{E}+00 \quad 0.000 \mathrm{E}+00$ $9.0000009536743 \mathrm{E}-01 \quad 1.0000000000000 \mathrm{E}+00 \quad 1.0000000000000 \mathrm{E}+00 \quad 0.000 \mathrm{E}+00$ $1.0000000000000 \mathrm{E}+00 \quad 0.0000000000000 \mathrm{E}+00 \quad 0.0000000000000 \mathrm{E}+00 \quad 0.000 \mathrm{E}+00$ $1.0000000000000 \mathrm{E}+00 \quad 0.0000000000000 \mathrm{E}+00 \quad 1.0000000000000 \mathrm{E}+00 \quad 0.000 \mathrm{E}+00$ $1.0000000000000 \mathrm{E}+001.0000000000000 \mathrm{E}+00 \quad 0.0000000000000 \mathrm{E}+00 \quad 0.000 \mathrm{E}+00$ $1.0000000000000 \mathrm{E}+00 \quad 1.0000000000000 \mathrm{E}+00 \quad 1.0000000000000 \mathrm{E}+00 \quad 0.000 \mathrm{E}+00$ $1.0000000000000 \mathrm{E}+00 \quad 0.0000000000000 \mathrm{E}+00 \quad 0.0000000000000 \mathrm{E}+00 \quad 0.000 \mathrm{E}+00$ $1.0000000000000 \mathrm{E}+00 \quad 0.0000000000000 \mathrm{E}+00 \quad 1.0000000000000 \mathrm{E}+00 \quad 0.000 \mathrm{E}+00$ $\begin{array}{lllll}1.0000000000000 \mathrm{E}+00 & 1.0000000000000 \mathrm{E}+00 & 0.0000000000000 \mathrm{E}+00 & 0.000 \mathrm{E}+00\end{array}$ $1.0000000000000 \mathrm{E}+001.0000000000000 \mathrm{E}+00 \quad 1.0000000000000 \mathrm{E}+00 \quad 0.000 \mathrm{E}+00$ $1.1000000238419 \mathrm{E}+00 \quad 0.0000000000000 \mathrm{E}+00 \quad 0.0000000000000 \mathrm{E}+00 \quad 0.000 \mathrm{E}+00$ $1.1000000238419 \mathrm{E}+00 \quad 0.0000000000000 \mathrm{E}+00 \quad 1.0000000000000 \mathrm{E}+00 \quad 0.000 \mathrm{E}+00$ $1.1000000238419 \mathrm{E}+00 \quad 1.0000000000000 \mathrm{E}+00 \quad 0.0000000000000 \mathrm{E}+00 \quad 0.000 \mathrm{E}+00$ $1.1000000238419 \mathrm{E}+00 \quad 1.0000000000000 \mathrm{E}+00 \quad 1.0000000000000 \mathrm{E}+00 \quad 0.000 \mathrm{E}+00$ $1.2000000476837 \mathrm{E}+00 \quad 0.0000000000000 \mathrm{E}+00 \quad 0.0000000000000 \mathrm{E}+00 \quad 0.000 \mathrm{E}+00$ $1.2000000476837 \mathrm{E}+00 \quad 0.0000000000000 \mathrm{E}+00 \quad 1.0000000000000 \mathrm{E}+00 \quad 0.000 \mathrm{E}+00$ $1.2000000476837 \mathrm{E}+00 \quad 1.0000000000000 \mathrm{E}+00 \quad 0.0000000000000 \mathrm{E}+00 \quad 0.000 \mathrm{E}+00$ $1.2000000476837 \mathrm{E}+00 \quad 1.0000000000000 \mathrm{E}+00 \quad 1.0000000000000 \mathrm{E}+00 \quad 0.000 \mathrm{E}+00$ $\begin{array}{lllll}1.2999999523163 \mathrm{E}+00 & 0.0000000000000 \mathrm{E}+00 & 0.0000000000000 \mathrm{E}+00 & 0.000 \mathrm{E}+00\end{array}$ $\begin{array}{llll}1.2999999523163 \mathrm{E}+00 & 0.0000000000000 \mathrm{E}+00 & 1.0000000000000 \mathrm{E}+00 \quad 0.000 \mathrm{E}+00\end{array}$ $\begin{array}{llll}1.2999999523163 \mathrm{E}+00 & 1.0000000000000 \mathrm{E}+00 & 0.0000000000000 \mathrm{E}+00 \quad 0.000 \mathrm{E}+00\end{array}$ $1.2999999523163 \mathrm{E}+00 \quad 1.0000000000000 \mathrm{E}+00 \quad 1.0000000000000 \mathrm{E}+00 \quad 0.000 \mathrm{E}+00$ $\begin{array}{llll}1.3999999761581 \mathrm{E}+00 & 0.0000000000000 \mathrm{E}+00 & 0.0000000000000 \mathrm{E}+00 & 0.000 \mathrm{E}+00\end{array}$ $1.3999999761581 \mathrm{E}+00 \quad 0.0000000000000 \mathrm{E}+00 \quad 1.0000000000000 \mathrm{E}+00 \quad 0.000 \mathrm{E}+00$ $\begin{array}{llll}1.3999999761581 \mathrm{E}+00 & 1.0000000000000 \mathrm{E}+00 & 0.0000000000000 \mathrm{E}+00 & 0.000 \mathrm{E}+00\end{array}$

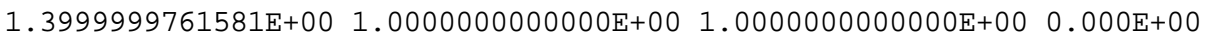
$1.5000000000000 \mathrm{E}+00 \quad 0.0000000000000 \mathrm{E}+00 \quad 0.0000000000000 \mathrm{E}+00 \quad 0.000 \mathrm{E}+00$ $1.5000000000000 \mathrm{E}+00 \quad 0.0000000000000 \mathrm{E}+00 \quad 1.0000000000000 \mathrm{E}+00 \quad 0.000 \mathrm{E}+00$ $1.5000000000000 \mathrm{E}+00 \quad 1.0000000000000 \mathrm{E}+00 \quad 0.0000000000000 \mathrm{E}+00 \quad 0.000 \mathrm{E}+00$ $1.5000000000000 \mathrm{E}+001.0000000000000 \mathrm{E}+00 \quad 1.0000000000000 \mathrm{E}+00 \quad 0.000 \mathrm{E}+00$ $1.6000000238419 \mathrm{E}+00 \quad 0.0000000000000 \mathrm{E}+00 \quad 0.0000000000000 \mathrm{E}+00 \quad 0.000 \mathrm{E}+00$ $1.6000000238419 \mathrm{E}+00 \quad 0.0000000000000 \mathrm{E}+00 \quad 1.0000000000000 \mathrm{E}+00 \quad 0.000 \mathrm{E}+00$ $1.6000000238419 \mathrm{E}+00 \quad 1.0000000000000 \mathrm{E}+00 \quad 0.0000000000000 \mathrm{E}+00 \quad 0.000 \mathrm{E}+00$ $1.6000000238419 \mathrm{E}+00 \quad 1.0000000000000 \mathrm{E}+00 \quad 1.0000000000000 \mathrm{E}+00 \quad 0.000 \mathrm{E}+00$ $1.7000000476837 \mathrm{E}+00 \quad 0.0000000000000 \mathrm{E}+00 \quad 0.0000000000000 \mathrm{E}+00 \quad 0.000 \mathrm{E}+00$ $1.7000000476837 \mathrm{E}+00 \quad 0.0000000000000 \mathrm{E}+00 \quad 1.0000000000000 \mathrm{E}+00 \quad 0.000 \mathrm{E}+00$ $1.7000000476837 \mathrm{E}+00 \quad 1.0000000000000 \mathrm{E}+00 \quad 0.0000000000000 \mathrm{E}+00 \quad 0.000 \mathrm{E}+00$ $1.7000000476837 \mathrm{E}+00 \quad 1.0000000000000 \mathrm{E}+00 \quad 1.0000000000000 \mathrm{E}+00 \quad 0.000 \mathrm{E}+00$ $1.8000000715256 \mathrm{E}+00 \quad 0.0000000000000 \mathrm{E}+00 \quad 0.0000000000000 \mathrm{E}+00 \quad 0.000 \mathrm{E}+00$ $1.8000000715256 \mathrm{E}+00 \quad 0.0000000000000 \mathrm{E}+00 \quad 1.0000000000000 \mathrm{E}+00 \quad 0.000 \mathrm{E}+00$ $1.8000000715256 \mathrm{E}+00 \quad 1.0000000000000 \mathrm{E}+00 \quad 0.0000000000000 \mathrm{E}+00 \quad 0.000 \mathrm{E}+00$ $1.8000000715256 \mathrm{E}+00 \quad 1.0000000000000 \mathrm{E}+00 \quad 1.0000000000000 \mathrm{E}+00 \quad 0.000 \mathrm{E}+00$ $\begin{array}{llll}1.9000000953674 \mathrm{E}+00 & 0.0000000000000 \mathrm{E}+00 & 0.0000000000000 \mathrm{E}+00 \quad 0.000 \mathrm{E}+00\end{array}$ $1.9000000953674 \mathrm{E}+00 \quad 0.0000000000000 \mathrm{E}+00 \quad 1.0000000000000 \mathrm{E}+00 \quad 0.000 \mathrm{E}+00$ 


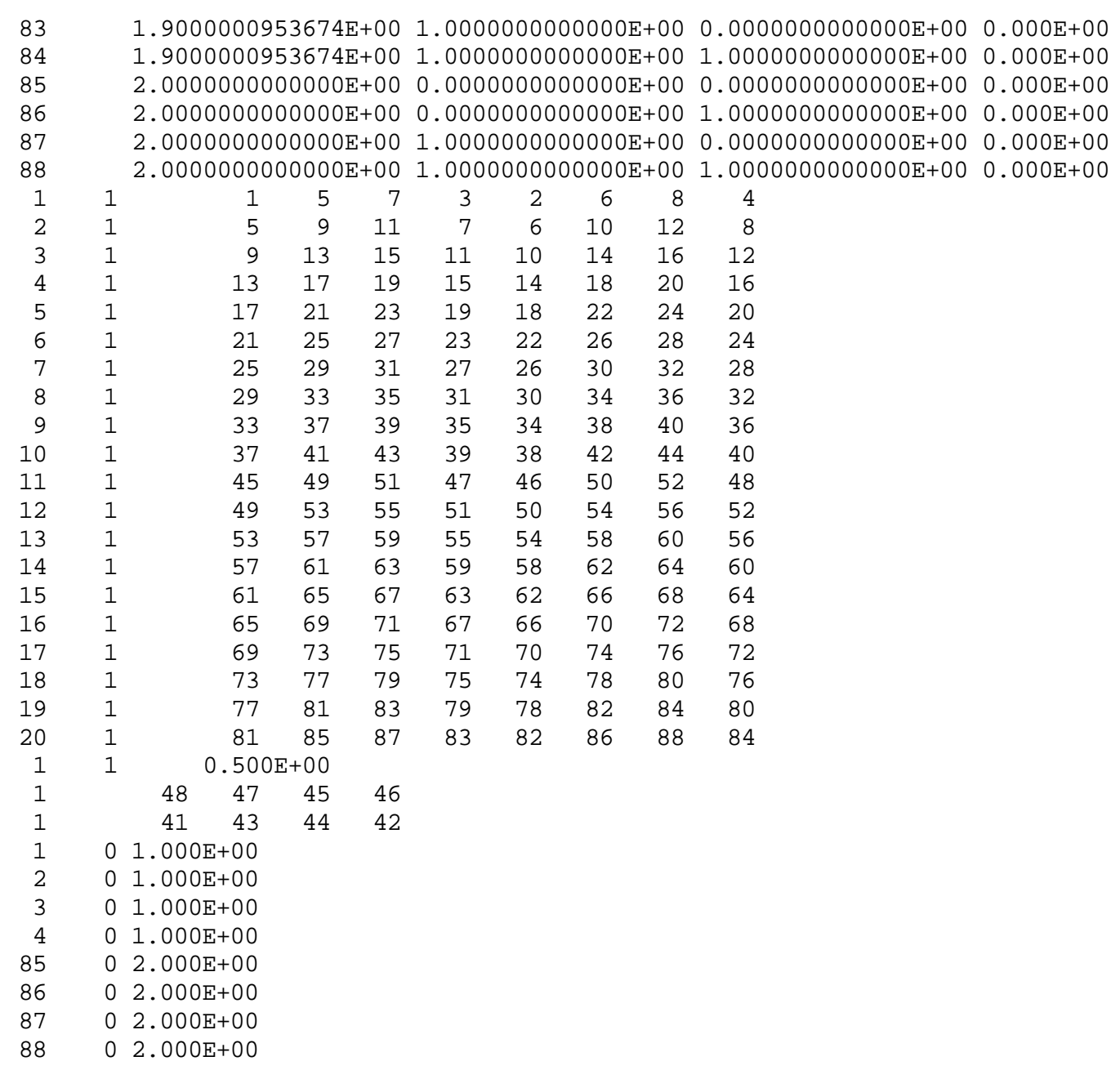

\section{$2.5 \quad$ Special Internal Element}

Problem: $\quad$ This models the steady linear flow of heat in two solids (each of length $\mathrm{L}$ ) separated by a gap with heat transfer occurring across the gap. In this problem temperature boundary conditions are specified at $\mathrm{x}=0$ (the left side of the first solid) and $\mathrm{x}=2 \mathrm{~L}+\mathrm{gap}$ thickness (the right side of the second solid). A thermal conductance $f$ across the gap is also specified. Note that the right side of the first solid is defined as surface A and the left side of the second solid as surface B.

Analytic solution: $\quad q^{\prime \prime}=f(T, t) \times\left(T_{s 1}{ }^{a}-T_{s 2}{ }^{a}\right)^{b}$, where $\mathrm{a}=\mathrm{b}=1$ for steady linear problem

Combined with the conduction equation, this yields 


$$
T_{B}=\frac{T_{L}\left(\frac{k}{L}+f\right)+f T_{0}}{\frac{k}{L}+2 f}, T_{A}=T_{L}+T_{0}-T_{B}
$$

$\begin{array}{lll}\text { Case parameters: } & \begin{array}{l}\text { Slab length - } \\ \text { Gap thickness - }\end{array} & \begin{array}{l}L=1 \text { (each slab) } \\ \text { gap }=0.1 \text { (this has no effect on the problem) }\end{array} \\ & \\ & \\ & \\ & f=0.5\end{array}$

Results: $\quad \underline{\text { Surface }} \quad \underline{T \text { (analytical) }} \quad \underline{T \text { (TOPAZ3D) }}$

$\begin{array}{lll}\mathrm{A} & 1.25 & 1.25 \\ \mathrm{~B} & 1.75 & 1.75\end{array}$

Note: The rest of the objects behaves under the laws governing onedimensional linear conduction, and the temperatures at various $\mathrm{x}$ coordinates can be determined and verified easily.

\section{Input file:}

C TOPAZ3D input file for steady linear internal element problem

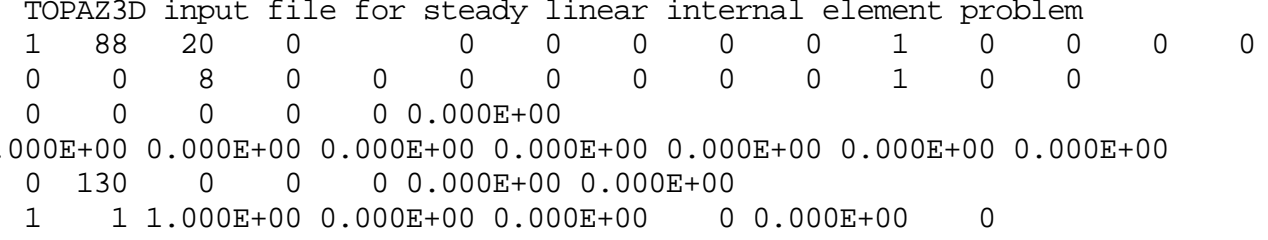

Material Type 1 - Isotropic

1.000E+00 1.000E+00

$10.0000000000000 \mathrm{E}+00 \quad 0.0000000000000 \mathrm{E}+00 \quad 0.0000000000000 \mathrm{E}+00 \quad 0.000 \mathrm{E}+00$

$2 \quad 0.0000000000000 \mathrm{E}+00 \quad 0.0000000000000 \mathrm{E}+00 \quad 1.0000000149012 \mathrm{E}-010.000 \mathrm{E}+00$

$30.0000000000000 \mathrm{E}+001.0000000149012 \mathrm{E}-010.0000000000000 \mathrm{E}+00 \quad 0.000 \mathrm{E}+00$

$40.0000000000000 \mathrm{E}+001.0000000149012 \mathrm{E}-011.0000000149012 \mathrm{E}-010.000 \mathrm{E}+00$

$5 \quad 1.0000000149012 \mathrm{E}-01 \quad 0.0000000000000 \mathrm{E}+00 \quad 0.0000000000000 \mathrm{E}+00 \quad 0.000 \mathrm{E}+00$

$6 \quad 1.0000000149012 \mathrm{E}-010.0000000000000 \mathrm{E}+00 \quad 1.0000000149012 \mathrm{E}-010.000 \mathrm{E}+00$

$7 \quad 1.0000000149012 \mathrm{E}-01 \quad 1.0000000149012 \mathrm{E}-010.0000000000000 \mathrm{E}+00 \quad 0.000 \mathrm{E}+00$

$8 \quad 1.0000000149012 \mathrm{E}-011.0000000149012 \mathrm{E}-011.0000000149012 \mathrm{E}-010.000 \mathrm{E}+00$

$92.0000000298023 \mathrm{E}-01 \quad 0.0000000000000 \mathrm{E}+00 \quad 0.0000000000000 \mathrm{E}+00 \quad 0.000 \mathrm{E}+00$

$102.0000000298023 \mathrm{E}-01 \quad 0.0000000000000 \mathrm{E}+00 \quad 1.0000000149012 \mathrm{E}-010.000 \mathrm{E}+00$

$112.0000000298023 \mathrm{E}-011.0000000149012 \mathrm{E}-010.0000000000000 \mathrm{E}+00 \quad 0.000 \mathrm{E}+00$

$2.0000000298023 \mathrm{E}-011.0000000149012 \mathrm{E}-01 \quad 1.0000000149012 \mathrm{E}-010.000 \mathrm{E}+00$

$3.0000001192093 \mathrm{E}-010.0000000000000 \mathrm{E}+00 \quad 0.0000000000000 \mathrm{E}+00 \quad 0.000 \mathrm{E}+00$

$3.0000001192093 \mathrm{E}-010.0000000000000 \mathrm{E}+00 \quad 1.0000000149012 \mathrm{E}-010.000 \mathrm{E}+00$

$3.0000001192093 \mathrm{E}-011.0000000149012 \mathrm{E}-01 \quad 0.0000000000000 \mathrm{E}+00 \quad 0.000 \mathrm{E}+00$

3.0000001192093E-01 1.0000000149012E-01 1.0000000149012E-01 0.000E+00

$\begin{array}{lllllll}4.0000000596046 \mathrm{E}-01 & 0.0000000000000 \mathrm{E}+00 & 0.0000000000000 \mathrm{E}+00 & 0.000 \mathrm{E}+00\end{array}$

$4.0000000596046 \mathrm{E}-01 \quad 0.0000000000000 \mathrm{E}+00 \quad 1.0000000149012 \mathrm{E}-010.000 \mathrm{E}+00$

$4.0000000596046 \mathrm{E}-01 \quad 1.0000000149012 \mathrm{E}-01 \quad 0.0000000000000 \mathrm{E}+00 \quad 0.000 \mathrm{E}+00$

4.0000000596046E-01 1.0000000149012E-01 1.0000000149012E-01 0.000E+00

$5.0000000000000 \mathrm{E}-010.0000000000000 \mathrm{E}+00 \quad 0.0000000000000 \mathrm{E}+00 \quad 0.000 \mathrm{E}+00$

$5.0000000000000 \mathrm{E}-010.0000000000000 \mathrm{E}+00 \quad 1.0000000149012 \mathrm{E}-010.000 \mathrm{E}+00$

$5.0000000000000 \mathrm{E}-01 \quad 1.0000000149012 \mathrm{E}-01 \quad 0.0000000000000 \mathrm{E}+00 \quad 0.000 \mathrm{E}+00$

$5.0000000000000 \mathrm{E}-011.0000000149012 \mathrm{E}-01 \quad 1.0000000149012 \mathrm{E}-010.000 \mathrm{E}+00$

$6.0000002384186 \mathrm{E}-01 \quad 0.0000000000000 \mathrm{E}+00 \quad 0.0000000000000 \mathrm{E}+00 \quad 0.000 \mathrm{E}+00$

$6.0000002384186 \mathrm{E}-010.0000000000000 \mathrm{E}+00 \quad 1.0000000149012 \mathrm{E}-010.000 \mathrm{E}+00$ 
$6.0000002384186 \mathrm{E}-01 \quad 1.0000000149012 \mathrm{E}-01 \quad 0.0000000000000 \mathrm{E}+00 \quad 0.000 \mathrm{E}+00$ $6.0000002384186 \mathrm{E}-01 \quad 1.0000000149012 \mathrm{E}-01 \quad 1.0000000149012 \mathrm{E}-010.000 \mathrm{E}+00$ $\begin{array}{lllll}7.0000004768372 \mathrm{E}-01 & 0.0000000000000 \mathrm{E}+00 & 0.0000000000000 \mathrm{E}+00 & 0.000 \mathrm{E}+00\end{array}$ $\begin{array}{llll}7.0000004768372 \mathrm{E}-01 & 0.0000000000000 \mathrm{E}+00 & 1.0000000149012 \mathrm{E}-01 \quad 0.000 \mathrm{E}+00\end{array}$ $\begin{array}{lllll}7.0000004768372 \mathrm{E}-01 & 1.0000000149012 \mathrm{E}-01 & 0.0000000000000 \mathrm{E}+00 & 0.000 \mathrm{E}+00\end{array}$ $7.0000004768372 \mathrm{E}-01 \quad 1.0000000149012 \mathrm{E}-01 \quad 1.0000000149012 \mathrm{E}-01 \quad 0.000 \mathrm{E}+00$ $\begin{array}{llllll}8.0000007152557 \mathrm{E}-01 & 0.0000000000000 \mathrm{E}+00 & 0.0000000000000 \mathrm{E}+00 & 0.000 \mathrm{E}+00\end{array}$ $8.0000007152557 \mathrm{E}-01 \quad 0.0000000000000 \mathrm{E}+00 \quad 1.0000000149012 \mathrm{E}-01 \quad 0.000 \mathrm{E}+00$ $8.0000007152557 \mathrm{E}-01 \quad 1.0000000149012 \mathrm{E}-01 \quad 0.0000000000000 \mathrm{E}+00 \quad 0.000 \mathrm{E}+00$ $8.0000007152557 \mathrm{E}-01 \quad 1.0000000149012 \mathrm{E}-01 \quad 1.0000000149012 \mathrm{E}-010.000 \mathrm{E}+00$ $9.0000009536743 \mathrm{E}-01 \quad 0.0000000000000 \mathrm{E}+00 \quad 0.0000000000000 \mathrm{E}+00 \quad 0.000 \mathrm{E}+00$ $9.0000009536743 \mathrm{E}-01 \quad 0.0000000000000 \mathrm{E}+00 \quad 1.0000000149012 \mathrm{E}-01 \quad 0.000 \mathrm{E}+00$ $9.0000009536743 \mathrm{E}-01 \quad 1.0000000149012 \mathrm{E}-01 \quad 0.0000000000000 \mathrm{E}+00 \quad 0.000 \mathrm{E}+00$ $9.0000009536743 \mathrm{E}-01 \quad 1.0000000149012 \mathrm{E}-01 \quad 1.0000000149012 \mathrm{E}-01 \quad 0.000 \mathrm{E}+00$ $1.0000000000000 \mathrm{E}+00 \quad 0.0000000000000 \mathrm{E}+00 \quad 0.0000000000000 \mathrm{E}+00 \quad 0.000 \mathrm{E}+00$ $1.0000000000000 \mathrm{E}+00 \quad 0.0000000000000 \mathrm{E}+00 \quad 1.0000000149012 \mathrm{E}-01 \quad 0.000 \mathrm{E}+00$ $1.0000000000000 \mathrm{E}+001.0000000149012 \mathrm{E}-01 \quad 0.0000000000000 \mathrm{E}+00 \quad 0.000 \mathrm{E}+00$ $1.0000000000000 \mathrm{E}+001.0000000149012 \mathrm{E}-01 \quad 1.0000000149012 \mathrm{E}-010.000 \mathrm{E}+00$ $\begin{array}{lllll}1.1000000238419 \mathrm{E}+00 & 0.0000000000000 \mathrm{E}+00 & 0.0000000000000 \mathrm{E}+00 & 0.000 \mathrm{E}+00\end{array}$ $1.1000000238419 \mathrm{E}+00 \quad 0.0000000000000 \mathrm{E}+00 \quad 1.0000000149012 \mathrm{E}-010.000 \mathrm{E}+00$ $1.1000000238419 \mathrm{E}+00 \quad 1.0000000149012 \mathrm{E}-01 \quad 0.0000000000000 \mathrm{E}+00 \quad 0.000 \mathrm{E}+00$ $1.1000000238419 \mathrm{E}+00 \quad 1.0000000149012 \mathrm{E}-01 \quad 1.0000000149012 \mathrm{E}-01 \quad 0.000 \mathrm{E}+00$ $1.2000000476837 \mathrm{E}+00 \quad 0.0000000000000 \mathrm{E}+00 \quad 0.0000000000000 \mathrm{E}+00 \quad 0.000 \mathrm{E}+00$ $\begin{array}{lllll}1.2000000476837 \mathrm{E}+00 & 0.0000000000000 \mathrm{E}+00 & 1.0000000149012 \mathrm{E}-01 & 0.000 \mathrm{E}+00\end{array}$ $1.2000000476837 \mathrm{E}+00 \quad 1.0000000149012 \mathrm{E}-01 \quad 0.0000000000000 \mathrm{E}+00 \quad 0.000 \mathrm{E}+00$ $1.2000000476837 \mathrm{E}+001.0000000149012 \mathrm{E}-01 \quad 1.0000000149012 \mathrm{E}-01 \quad 0.000 \mathrm{E}+00$ $\begin{array}{llll}1.2999999523163 \mathrm{E}+00 & 0.0000000000000 \mathrm{E}+00 & 0.0000000000000 \mathrm{E}+00 & 0.000 \mathrm{E}+00\end{array}$ $\begin{array}{lllll}1.2999999523163 \mathrm{E}+00 & 0.0000000000000 \mathrm{E}+00 & 1.0000000149012 \mathrm{E}-01 & 0.000 \mathrm{E}+00\end{array}$ $\begin{array}{llllll}1.2999999523163 \mathrm{E}+00 & 1.0000000149012 \mathrm{E}-01 & 0.0000000000000 \mathrm{E}+00 & 0.000 \mathrm{E}+00\end{array}$ $1.2999999523163 \mathrm{E}+001.0000000149012 \mathrm{E}-01 \quad 1.0000000149012 \mathrm{E}-010.000 \mathrm{E}+00$ $\begin{array}{llll}1.3999999761581 \mathrm{E}+00 & 0.0000000000000 \mathrm{E}+00 & 0.0000000000000 \mathrm{E}+00 & 0.000 \mathrm{E}+00\end{array}$ $\begin{array}{lllll}1.3999999761581 \mathrm{E}+00 & 0.0000000000000 \mathrm{E}+00 & 1.0000000149012 \mathrm{E}-01 \quad 0.000 \mathrm{E}+00\end{array}$ $\begin{array}{lllll}1.3999999761581 \mathrm{E}+00 & 1.0000000149012 \mathrm{E}-01 & 0.0000000000000 \mathrm{E}+00 & 0.000 \mathrm{E}+00\end{array}$ $1.3999999761581 \mathrm{E}+001.0000000149012 \mathrm{E}-01 \quad 1.0000000149012 \mathrm{E}-01 \quad 0.000 \mathrm{E}+00$ $1.5000000000000 \mathrm{E}+00 \quad 0.0000000000000 \mathrm{E}+00 \quad 0.0000000000000 \mathrm{E}+00 \quad 0.000 \mathrm{E}+00$ $1.5000000000000 \mathrm{E}+00 \quad 0.0000000000000 \mathrm{E}+00 \quad 1.0000000149012 \mathrm{E}-01 \quad 0.000 \mathrm{E}+00$ $1.5000000000000 \mathrm{E}+00 \quad 1.0000000149012 \mathrm{E}-01 \quad 0.0000000000000 \mathrm{E}+00 \quad 0.000 \mathrm{E}+00$ $1.5000000000000 \mathrm{E}+001.0000000149012 \mathrm{E}-01 \quad 1.0000000149012 \mathrm{E}-010.000 \mathrm{E}+00$ $\begin{array}{llllll}1.5999999046326 \mathrm{E}+00 & 0.0000000000000 \mathrm{E}+00 & 0.0000000000000 \mathrm{E}+00 & 0.000 \mathrm{E}+00\end{array}$ $1.5999999046326 \mathrm{E}+00 \quad 0.0000000000000 \mathrm{E}+00 \quad 1.0000000149012 \mathrm{E}-01 \quad 0.000 \mathrm{E}+00$ $\begin{array}{llll}1.5999999046326 \mathrm{E}+00 & 1.0000000149012 \mathrm{E}-01 & 0.0000000000000 \mathrm{E}+00 & 0.000 \mathrm{E}+00\end{array}$ $1.5999999046326 \mathrm{E}+00 \quad 1.0000000149012 \mathrm{E}-01 \quad 1.0000000149012 \mathrm{E}-01 \quad 0.000 \mathrm{E}+00$ $1.7000000476837 \mathrm{E}+00 \quad 0.0000000000000 \mathrm{E}+00 \quad 0.0000000000000 \mathrm{E}+00 \quad 0.000 \mathrm{E}+00$ $1.7000000476837 \mathrm{E}+00 \quad 0.0000000000000 \mathrm{E}+00 \quad 1.0000000149012 \mathrm{E}-01 \quad 0.000 \mathrm{E}+00$ $1.7000000476837 \mathrm{E}+001.0000000149012 \mathrm{E}-010.0000000000000 \mathrm{E}+00 \quad 0.000 \mathrm{E}+00$ $1.7000000476837 \mathrm{E}+001.0000000149012 \mathrm{E}-01 \quad 1.0000000149012 \mathrm{E}-01 \quad 0.000 \mathrm{E}+00$

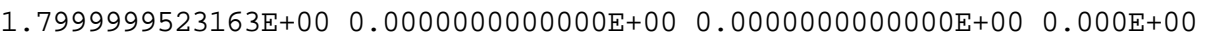
$1.7999999523163 \mathrm{E}+00 \quad 0.0000000000000 \mathrm{E}+00 \quad 1.0000000149012 \mathrm{E}-010.000 \mathrm{E}+00$ $1.7999999523163 \mathrm{E}+00 \quad 1.0000000149012 \mathrm{E}-01 \quad 0.0000000000000 \mathrm{E}+00 \quad 0.000 \mathrm{E}+00$ $1.7999999523163 \mathrm{E}+00 \quad 1.0000000149012 \mathrm{E}-01 \quad 1.0000000149012 \mathrm{E}-01 \quad 0.000 \mathrm{E}+00$ $\begin{array}{llll}1.8999999761581 \mathrm{E}+00 & 0.0000000000000 \mathrm{E}+00 & 0.0000000000000 \mathrm{E}+00 & 0.000 \mathrm{E}+00\end{array}$ $\begin{array}{llll}1.8999999761581 \mathrm{E}+00 & 0.0000000000000 \mathrm{E}+00 & 1.0000000149012 \mathrm{E}-01 \quad 0.000 \mathrm{E}+00\end{array}$ $\begin{array}{lllll}1.8999999761581 \mathrm{E}+00 & 1.0000000149012 \mathrm{E}-01 & 0.0000000000000 \mathrm{E}+00 & 0.000 \mathrm{E}+00\end{array}$ $1.8999999761581 \mathrm{E}+001.0000000149012 \mathrm{E}-01 \quad 1.0000000149012 \mathrm{E}-01 \quad 0.000 \mathrm{E}+00$ $2.0000000000000 \mathrm{E}+00 \quad 0.0000000000000 \mathrm{E}+00 \quad 0.0000000000000 \mathrm{E}+00 \quad 0.000 \mathrm{E}+00$ $2.0000000000000 \mathrm{E}+00 \quad 0.0000000000000 \mathrm{E}+00 \quad 1.0000000149012 \mathrm{E}-01 \quad 0.000 \mathrm{E}+00$ $2.0000000000000 \mathrm{E}+00 \quad 1.0000000149012 \mathrm{E}-01 \quad 0.0000000000000 \mathrm{E}+00 \quad 0.000 \mathrm{E}+00$ $2.0000000000000 \mathrm{E}+001.0000000149012 \mathrm{E}-01 \quad 1.0000000149012 \mathrm{E}-01 \quad 0.000 \mathrm{E}+00$ $2.0999999046326 \mathrm{E}+00 \quad 0.0000000000000 \mathrm{E}+00 \quad 0.0000000000000 \mathrm{E}+00 \quad 0.000 \mathrm{E}+00$ $2.0999999046326 \mathrm{E}+00 \quad 0.0000000000000 \mathrm{E}+00 \quad 1.0000000149012 \mathrm{E}-01 \quad 0.000 \mathrm{E}+00$ $2.0999999046326 \mathrm{E}+00 \quad 1.0000000149012 \mathrm{E}-01 \quad 0.0000000000000 \mathrm{E}+00 \quad 0.000 \mathrm{E}+00$ $2.0999999046326 \mathrm{E}+00 \quad 1.0000000149012 \mathrm{E}-01 \quad 1.0000000149012 \mathrm{E}-01 \quad 0.000 \mathrm{E}+00$ 1

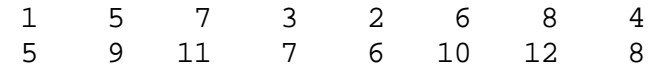




\begin{tabular}{|c|c|c|c|c|c|c|c|c|}
\hline 3 & 1 & 9 & 13 & 15 & 11 & 10 & 14 & 16 \\
\hline 4 & 1 & 13 & 17 & 19 & 15 & 14 & 18 & 20 \\
\hline 5 & 1 & 17 & 21 & 23 & 19 & 18 & 22 & 24 \\
\hline 6 & 1 & 21 & 25 & 27 & 23 & 22 & 26 & 28 \\
\hline 7 & 1 & 25 & 29 & 31 & 27 & 26 & 30 & 32 \\
\hline 8 & 1 & 29 & 33 & 35 & 31 & 30 & 34 & 36 \\
\hline 9 & 1 & 33 & 37 & 39 & 35 & 34 & 38 & 40 \\
\hline 10 & 1 & 37 & 41 & 43 & 39 & 38 & 42 & 44 \\
\hline 11 & 1 & 45 & 49 & 51 & 47 & 46 & 50 & 52 \\
\hline 12 & 1 & 49 & 53 & 55 & 51 & 50 & 54 & 56 \\
\hline 13 & 1 & 53 & 57 & 59 & 55 & 54 & 58 & 60 \\
\hline 14 & 1 & 57 & 61 & 63 & 59 & 58 & 62 & 64 \\
\hline 15 & 1 & 61 & 65 & 67 & 63 & 62 & 66 & 68 \\
\hline 16 & 1 & 65 & 69 & 71 & 67 & 66 & 70 & 72 \\
\hline 17 & 1 & 69 & 73 & 75 & 71 & 70 & 74 & 76 \\
\hline 18 & 1 & 73 & 77 & 79 & 75 & 74 & 78 & 80 \\
\hline 19 & 1 & 77 & 81 & 83 & 79 & 78 & 82 & 84 \\
\hline 20 & 1 & 81 & 85 & 87 & 83 & 82 & 86 & 88 \\
\hline 1 & 0 & $1.000 \mathrm{E}+00$ & & & & & & \\
\hline 2 & 0 & $1.000 \mathrm{E}+00$ & & & & & & \\
\hline 3 & 0 & $1.000 \mathrm{E}+00$ & & & & & & \\
\hline 4 & 0 & $1.000 \mathrm{E}+00$ & & & & & & \\
\hline 85 & 0 & $2.000 E+00$ & & & & & & \\
\hline 86 & 0 & $2.000 \mathrm{E}+00$ & & & & & & \\
\hline 87 & 0 & $2.000 E+00$ & & & & & & \\
\hline 88 & 0 & $2.000 \mathrm{E}+00$ & & & & & & \\
\hline 41 & 43 & $44 \quad 42$ & 45 & 47 & 48 & 46 & & \\
\hline
\end{tabular}

\subsection{Transient Linear Problems}

The problems in this section model the transient, linear flow of heat. The length $\mathrm{L}$ for this set of problems is either 1.0 or 10.0. The first three problems in this section (constant temperature, flux and convection boundary conditions) are examined in a fashion similar to that of the steady problems: the temperatures at various x-coordinate locations are examined after a time interval t. The next two problems (slide line and internal element) are examined by comparing the solution at the points of interest with the solution produced by assuming the problem to be that of simple transient conduction with a constant temperature boundary condition. This is explained further in those particular problems. The last three problems in this section (temporal temperature, flux and convection boundary conditions) are examined by looking at the temperatures of only one $\mathrm{x}$-coordinate location at various times.

The problems represented in this section are: 
3.1 - Constant Temperature Boundary Condition

3.2 - Constant Flux Boundary Condition

3.3 - Constant Convection Boundary Condition

3.4 - Constant Slide Line

3.5 - Constant Special Internal Element

3.6 - Temporal Temperature Boundary Condition

3.7 - Temporal Flux Boundary Condition

3.8 - Temporal Convection Boundary Condition

\subsection{Constant Temperature Boundary Condition}

Problem: $\quad$ This case models the transient flow of heat in a semi-infinite solid with a temperature boundary condition set at $\mathrm{x}=0$, a global initial temperature condition, and a set time interval.

Analytic solution: $\quad T_{x}=\left(T_{i}-T_{0}\right) \times \operatorname{erf}\left(\frac{x}{2 \sqrt{\alpha t}}\right)+T_{0} \quad$ (Incropera \& DeWitt, p.239)

Case parameters: $\quad$ Slab length - $\quad L=10$

Boundary conditions $-T_{0}=0$ at $x=0$

Time -

$T_{i}=10$

$t=3$

Results: $\quad \underline{\text { x-coordinate }} \underline{T \text { (analytical) }} \underline{T(T O P A Z 3 D)}$

$\begin{array}{rrr}0.00 & 0.00000 & 0.00000 \\ 1.00 & 3.16909 & 3.17056 \\ 2.00 & 5.85784 & 5.85856 \\ 3.00 & 7.79329 & 7.79136 \\ 4.00 & 8.97530 & 8.97104 \\ 5.00 & 9.58773 & 9.58300 \\ 6.00 & 9.85694 & 9.85331 \\ 7.00 & 9.95733 & 9.95522 \\ 8.00 & 9.98909 & 9.98811 \\ 9.00 & 9.99762 & 9.99716 \\ 10.00 & 9.99955 & 9.99899\end{array}$

Input file:

C TOPAZ3D input file for transient linear temperature boundary condition problem

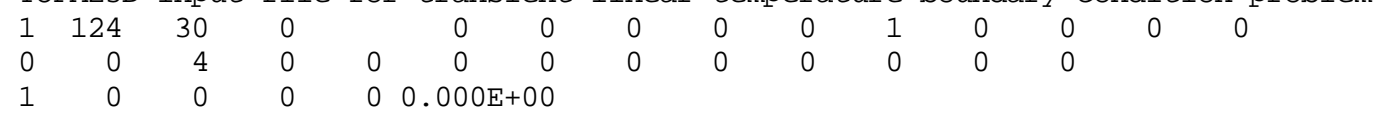


$\begin{array}{llllllll}0.000 \mathrm{E}+00 & 3.000 \mathrm{E}+00 & 1.000 \mathrm{E}-01 & 0.000 \mathrm{E}+00 & 0.000 \mathrm{E}+00 & 0.000 \mathrm{E}+00 & 0.000 \mathrm{E}+00\end{array}$

$\begin{array}{lllllll}0 & 0 & 0 & 0 & 0 & 0.000 \mathrm{E}+00 & 0.000 \mathrm{E}+00\end{array}$

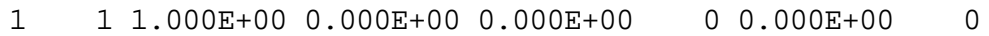

Material Type 1 - Isotropic

$1.000 \mathrm{E}+001.000 \mathrm{E}+00$

$1 \quad 0.0000000000000 \mathrm{E}+00 \quad 0.0000000000000 \mathrm{E}+00 \quad 0.0000000000000 \mathrm{E}+00 \quad 1.000 \mathrm{E}+01$

$2 \quad 0.0000000000000 \mathrm{E}+00 \quad 0.0000000000000 \mathrm{E}+00 \quad 1.0000000000000 \mathrm{E}+00 \quad 1.000 \mathrm{E}+01$

$3 \quad 0.0000000000000 \mathrm{E}+00 \quad 1.0000000000000 \mathrm{E}+00 \quad 0.0000000000000 \mathrm{E}+001.000 \mathrm{E}+01$

$4 \quad 0.0000000000000 \mathrm{E}+001.0000000000000 \mathrm{E}+00 \quad 1.0000000000000 \mathrm{E}+001.000 \mathrm{E}+01$

$5 \quad 3.3333334326744 \mathrm{E}-01 \quad 0.0000000000000 \mathrm{E}+00 \quad 0.0000000000000 \mathrm{E}+00 \quad 1.000 \mathrm{E}+01$

$6 \quad 3.3333334326744 \mathrm{E}-01 \quad 0.0000000000000 \mathrm{E}+00 \quad 1.0000000000000 \mathrm{E}+00 \quad 1.000 \mathrm{E}+01$

$7 \quad 3.3333334326744 \mathrm{E}-01 \quad 1.0000000000000 \mathrm{E}+00 \quad 0.0000000000000 \mathrm{E}+00 \quad 1.000 \mathrm{E}+01$

$8 \quad 3.3333334326744 \mathrm{E}-01 \quad 1.0000000000000 \mathrm{E}+00 \quad 1.0000000000000 \mathrm{E}+001.000 \mathrm{E}+01$

$96.6666668653488 \mathrm{E}-01 \quad 0.0000000000000 \mathrm{E}+00 \quad 0.0000000000000 \mathrm{E}+00 \quad 1.000 \mathrm{E}+01$

$106.6666668653488 \mathrm{E}-01 \quad 0.0000000000000 \mathrm{E}+00 \quad 1.0000000000000 \mathrm{E}+00 \quad 1.000 \mathrm{E}+01$

$116.6666668653488 \mathrm{E}-01 \quad 1.0000000000000 \mathrm{E}+00 \quad 0.0000000000000 \mathrm{E}+00 \quad 1.000 \mathrm{E}+01$

$126.6666668653488 \mathrm{E}-01 \quad 1.0000000000000 \mathrm{E}+00 \quad 1.0000000000000 \mathrm{E}+001.000 \mathrm{E}+01$

$131.0000001192093 \mathrm{E}+00 \quad 0.0000000000000 \mathrm{E}+00 \quad 0.0000000000000 \mathrm{E}+00 \quad 1.000 \mathrm{E}+01$

$141.0000001192093 \mathrm{E}+00 \quad 0.0000000000000 \mathrm{E}+00 \quad 1.0000000000000 \mathrm{E}+00 \quad 1.000 \mathrm{E}+01$

$151.0000001192093 \mathrm{E}+001.0000000000000 \mathrm{E}+00 \quad 0.0000000000000 \mathrm{E}+001.000 \mathrm{E}+01$

$161.0000001192093 \mathrm{E}+001.0000000000000 \mathrm{E}+00 \quad 1.0000000000000 \mathrm{E}+001.000 \mathrm{E}+01$

$1.3333333730698 \mathrm{E}+00 \quad 0.0000000000000 \mathrm{E}+00 \quad 0.0000000000000 \mathrm{E}+00 \quad 1.000 \mathrm{E}+01$ $1.3333333730698 \mathrm{E}+00 \quad 0.0000000000000 \mathrm{E}+00 \quad 1.0000000000000 \mathrm{E}+00 \quad 1.000 \mathrm{E}+01$

$1.3333333730698 \mathrm{E}+00 \quad 1.0000000000000 \mathrm{E}+00 \quad 0.0000000000000 \mathrm{E}+00 \quad 1.000 \mathrm{E}+01$ $1.3333333730698 \mathrm{E}+00 \quad 1.0000000000000 \mathrm{E}+00 \quad 1.0000000000000 \mathrm{E}+00 \quad 1.000 \mathrm{E}+01$ $\begin{array}{llll}1.6666667461395 \mathrm{E}+00 & 0.0000000000000 \mathrm{E}+00 & 0.0000000000000 \mathrm{E}+00 & 1.000 \mathrm{E}+01\end{array}$ $1.6666667461395 \mathrm{E}+00 \quad 0.0000000000000 \mathrm{E}+00 \quad 1.0000000000000 \mathrm{E}+00 \quad 1.000 \mathrm{E}+01$ $\begin{array}{llll}1.6666667461395 \mathrm{E}+00 & 1.0000000000000 \mathrm{E}+00 & 0.0000000000000 \mathrm{E}+00 & 1.000 \mathrm{E}+01\end{array}$

$\begin{array}{llll}1.6666667461395 \mathrm{E}+00 & 1.0000000000000 \mathrm{E}+00 & 1.0000000000000 \mathrm{E}+00 & 1.000 \mathrm{E}+01\end{array}$ $2.0000000000000 \mathrm{E}+00 \quad 0.0000000000000 \mathrm{E}+00 \quad 0.0000000000000 \mathrm{E}+00 \quad 1.000 \mathrm{E}+01$ $2.0000000000000 \mathrm{E}+00 \quad 0.0000000000000 \mathrm{E}+00 \quad 1.0000000000000 \mathrm{E}+00 \quad 1.000 \mathrm{E}+01$ $2.0000000000000 \mathrm{E}+00 \quad 1.0000000000000 \mathrm{E}+00 \quad 0.0000000000000 \mathrm{E}+00 \quad 1.000 \mathrm{E}+01$ $2.0000000000000 \mathrm{E}+001.0000000000000 \mathrm{E}+00 \quad 1.0000000000000 \mathrm{E}+00 \quad 1.000 \mathrm{E}+01$ $\begin{array}{llll}2.3333332538605 \mathrm{E}+00 & 0.0000000000000 \mathrm{E}+00 & 0.0000000000000 \mathrm{E}+00 & 1.000 \mathrm{E}+01\end{array}$ $2.3333332538605 \mathrm{E}+00 \quad 0.0000000000000 \mathrm{E}+00 \quad 1.0000000000000 \mathrm{E}+00 \quad 1.000 \mathrm{E}+01$ $2.3333332538605 \mathrm{E}+00 \quad 1.0000000000000 \mathrm{E}+00 \quad 0.0000000000000 \mathrm{E}+00 \quad 1.000 \mathrm{E}+01$ $2.3333332538605 \mathrm{E}+001.0000000000000 \mathrm{E}+00 \quad 1.0000000000000 \mathrm{E}+00 \quad 1.000 \mathrm{E}+01$ $\begin{array}{llll}2.6666667461395 \mathrm{E}+00 & 0.0000000000000 \mathrm{E}+00 & 0.0000000000000 \mathrm{E}+00 & 1.000 \mathrm{E}+01\end{array}$ $2.6666667461395 \mathrm{E}+00 \quad 0.0000000000000 \mathrm{E}+00 \quad 1.0000000000000 \mathrm{E}+00 \quad 1.000 \mathrm{E}+01$ $2.6666667461395 \mathrm{E}+00 \quad 1.0000000000000 \mathrm{E}+00 \quad 0.0000000000000 \mathrm{E}+00 \quad 1.000 \mathrm{E}+01$ $2.6666667461395 \mathrm{E}+00 \quad 1.0000000000000 \mathrm{E}+00 \quad 1.0000000000000 \mathrm{E}+00 \quad 1.000 \mathrm{E}+01$ $3.0000000000000 \mathrm{E}+00 \quad 0.0000000000000 \mathrm{E}+00 \quad 0.0000000000000 \mathrm{E}+00 \quad 1.000 \mathrm{E}+01$ 3.0000000000000E+00 $0.0000000000000 \mathrm{E}+00 \quad 1.0000000000000 \mathrm{E}+00 \quad 1.000 \mathrm{E}+01$ $3.0000000000000 \mathrm{E}+00 \quad 1.0000000000000 \mathrm{E}+00 \quad 0.0000000000000 \mathrm{E}+00 \quad 1.000 \mathrm{E}+01$ $3.0000000000000 \mathrm{E}+001.0000000000000 \mathrm{E}+00 \quad 1.0000000000000 \mathrm{E}+00 \quad 1.000 \mathrm{E}+01$ $\begin{array}{llll}3.3333334922791 \mathrm{E}+00 & 0.0000000000000 \mathrm{E}+00 & 0.0000000000000 \mathrm{E}+00 & 1.000 \mathrm{E}+01\end{array}$ $3.3333334922791 \mathrm{E}+00 \quad 0.0000000000000 \mathrm{E}+00 \quad 1.0000000000000 \mathrm{E}+00 \quad 1.000 \mathrm{E}+01$ $3.3333334922791 \mathrm{E}+00 \quad 1.0000000000000 \mathrm{E}+00 \quad 0.0000000000000 \mathrm{E}+00 \quad 1.000 \mathrm{E}+01$ $3.3333334922791 \mathrm{E}+00 \quad 1.0000000000000 \mathrm{E}+00 \quad 1.0000000000000 \mathrm{E}+00 \quad 1.000 \mathrm{E}+01$ $\begin{array}{llll}3.6666667461395 \mathrm{E}+00 & 0.0000000000000 \mathrm{E}+00 & 0.0000000000000 \mathrm{E}+00 & 1.000 \mathrm{E}+01\end{array}$ $3.6666667461395 \mathrm{E}+00 \quad 0.0000000000000 \mathrm{E}+00 \quad 1.0000000000000 \mathrm{E}+00 \quad 1.000 \mathrm{E}+01$ $3.6666667461395 \mathrm{E}+00 \quad 1.0000000000000 \mathrm{E}+00 \quad 0.0000000000000 \mathrm{E}+00 \quad 1.000 \mathrm{E}+01$ $3.6666667461395 \mathrm{E}+00 \quad 1.0000000000000 \mathrm{E}+00 \quad 1.0000000000000 \mathrm{E}+00 \quad 1.000 \mathrm{E}+01$ $4.0000000000000 \mathrm{E}+00 \quad 0.0000000000000 \mathrm{E}+00 \quad 0.0000000000000 \mathrm{E}+00 \quad 1.000 \mathrm{E}+01$ $4.0000000000000 \mathrm{E}+00 \quad 0.0000000000000 \mathrm{E}+00 \quad 1.0000000000000 \mathrm{E}+00 \quad 1.000 \mathrm{E}+01$ $4.0000000000000 \mathrm{E}+00 \quad 1.0000000000000 \mathrm{E}+00 \quad 0.0000000000000 \mathrm{E}+00 \quad 1.000 \mathrm{E}+01$ $4.0000000000000 \mathrm{E}+00 \quad 1.0000000000000 \mathrm{E}+00 \quad 1.0000000000000 \mathrm{E}+00 \quad 1.000 \mathrm{E}+01$ $\begin{array}{llll}4.3333334922791 \mathrm{E}+00 & 0.0000000000000 \mathrm{E}+00 & 0.0000000000000 \mathrm{E}+00 \quad 1.000 \mathrm{E}+01\end{array}$ $\begin{array}{llll}4.3333334922791 \mathrm{E}+00 & 0.0000000000000 \mathrm{E}+00 & 1.0000000000000 \mathrm{E}+00 & 1.000 \mathrm{E}+01\end{array}$ $\begin{array}{llll}4.3333334922791 \mathrm{E}+00 & 1.0000000000000 \mathrm{E}+00 & 0.0000000000000 \mathrm{E}+00 & 1.000 \mathrm{E}+01\end{array}$ $4.3333334922791 \mathrm{E}+00 \quad 1.0000000000000 \mathrm{E}+00 \quad 1.0000000000000 \mathrm{E}+00 \quad 1.000 \mathrm{E}+01$ $\begin{array}{lllll}4.6666665077209 \mathrm{E}+00 & 0.0000000000000 \mathrm{E}+00 & 0.0000000000000 \mathrm{E}+00 & 1.000 \mathrm{E}+01\end{array}$ $\begin{array}{llll}4.6666665077209 \mathrm{E}+00 & 0.0000000000000 \mathrm{E}+00 & 1.0000000000000 \mathrm{E}+00 \quad 1.000 \mathrm{E}+01\end{array}$ $\begin{array}{llll}4.6666665077209 \mathrm{E}+00 & 1.0000000000000 \mathrm{E}+00 & 0.0000000000000 \mathrm{E}+00 & 1.000 \mathrm{E}+01\end{array}$ 


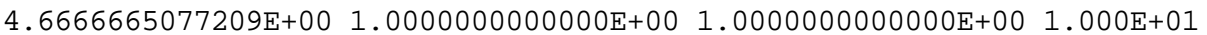
$5.0000000000000 \mathrm{E}+00 \quad 0.0000000000000 \mathrm{E}+00 \quad 0.0000000000000 \mathrm{E}+00 \quad 1.000 \mathrm{E}+01$ $5.0000000000000 \mathrm{E}+00 \quad 0.0000000000000 \mathrm{E}+00 \quad 1.0000000000000 \mathrm{E}+00 \quad 1.000 \mathrm{E}+01$ $5.0000000000000 \mathrm{E}+00 \quad 1.0000000000000 \mathrm{E}+00 \quad 0.0000000000000 \mathrm{E}+00 \quad 1.000 \mathrm{E}+01$ $5.0000000000000 \mathrm{E}+001.0000000000000 \mathrm{E}+00 \quad 1.0000000000000 \mathrm{E}+001.000 \mathrm{E}+01$ $\begin{array}{llll}5.3333334922791 \mathrm{E}+00 & 0.0000000000000 \mathrm{E}+00 & 0.0000000000000 \mathrm{E}+00 & 1.000 \mathrm{E}+01\end{array}$ $5.3333334922791 \mathrm{E}+00 \quad 0.0000000000000 \mathrm{E}+00 \quad 1.0000000000000 \mathrm{E}+00 \quad 1.000 \mathrm{E}+01$ $5.3333334922791 \mathrm{E}+001.0000000000000 \mathrm{E}+00 \quad 0.0000000000000 \mathrm{E}+00 \quad 1.000 \mathrm{E}+01$ $\begin{array}{llll}5.3333334922791 \mathrm{E}+00 & 1.0000000000000 \mathrm{E}+00 & 1.0000000000000 \mathrm{E}+00 & 1.000 \mathrm{E}+01\end{array}$ $\begin{array}{llll}5.6666669845581 \mathrm{E}+00 & 0.0000000000000 \mathrm{E}+00 & 0.0000000000000 \mathrm{E}+00 & 1.000 \mathrm{E}+01\end{array}$ $\begin{array}{llll}5.6666669845581 \mathrm{E}+00 & 0.0000000000000 \mathrm{E}+00 & 1.0000000000000 \mathrm{E}+00 & 1.000 \mathrm{E}+01\end{array}$ $5.6666669845581 \mathrm{E}+00 \quad 1.0000000000000 \mathrm{E}+00 \quad 0.0000000000000 \mathrm{E}+00 \quad 1.000 \mathrm{E}+01$ $5.6666669845581 \mathrm{E}+00 \quad 1.0000000000000 \mathrm{E}+00 \quad 1.0000000000000 \mathrm{E}+00 \quad 1.000 \mathrm{E}+01$ $6.0000009536743 \mathrm{E}+00 \quad 0.0000000000000 \mathrm{E}+00 \quad 0.0000000000000 \mathrm{E}+00 \quad 1.000 \mathrm{E}+01$ $6.0000009536743 \mathrm{E}+00 \quad 0.0000000000000 \mathrm{E}+00 \quad 1.0000000000000 \mathrm{E}+00 \quad 1.000 \mathrm{E}+01$ $6.0000009536743 \mathrm{E}+00 \quad 1.0000000000000 \mathrm{E}+00 \quad 0.0000000000000 \mathrm{E}+00 \quad 1.000 \mathrm{E}+01$ $6.0000009536743 \mathrm{E}+001.0000000000000 \mathrm{E}+00 \quad 1.0000000000000 \mathrm{E}+00 \quad 1.000 \mathrm{E}+01$ $6.3333344459534 \mathrm{E}+00 \quad 0.0000000000000 \mathrm{E}+00 \quad 0.0000000000000 \mathrm{E}+00 \quad 1.000 \mathrm{E}+01$ $\begin{array}{llll}6.3333344459534 \mathrm{E}+00 & 0.0000000000000 \mathrm{E}+00 & 1.0000000000000 \mathrm{E}+00 & 1.000 \mathrm{E}+01\end{array}$ $6.3333344459534 \mathrm{E}+00 \quad 1.0000000000000 \mathrm{E}+00 \quad 0.0000000000000 \mathrm{E}+00 \quad 1.000 \mathrm{E}+01$ $6.3333344459534 \mathrm{E}+00 \quad 1.0000000000000 \mathrm{E}+00 \quad 1.0000000000000 \mathrm{E}+00 \quad 1.000 \mathrm{E}+01$ $\begin{array}{llll}6.6666679382324 \mathrm{E}+00 & 0.0000000000000 \mathrm{E}+00 & 0.0000000000000 \mathrm{E}+00 & 1.000 \mathrm{E}+01\end{array}$ $6.6666679382324 \mathrm{E}+00 \quad 0.0000000000000 \mathrm{E}+00 \quad 1.0000000000000 \mathrm{E}+00 \quad 1.000 \mathrm{E}+01$ $6.6666679382324 \mathrm{E}+00 \quad 1.0000000000000 \mathrm{E}+00 \quad 0.0000000000000 \mathrm{E}+00 \quad 1.000 \mathrm{E}+01$ $6.6666679382324 \mathrm{E}+00 \quad 1.0000000000000 \mathrm{E}+00 \quad 1.0000000000000 \mathrm{E}+00 \quad 1.000 \mathrm{E}+01$ $\begin{array}{llll}7.0000019073486 \mathrm{E}+00 & 0.0000000000000 \mathrm{E}+00 & 0.0000000000000 \mathrm{E}+00 \quad 1.000 \mathrm{E}+01\end{array}$ $7.0000019073486 \mathrm{E}+00 \quad 0.0000000000000 \mathrm{E}+00 \quad 1.0000000000000 \mathrm{E}+00 \quad 1.000 \mathrm{E}+01$ $7.0000019073486 \mathrm{E}+00 \quad 1.0000000000000 \mathrm{E}+00 \quad 0.0000000000000 \mathrm{E}+00 \quad 1.000 \mathrm{E}+01$

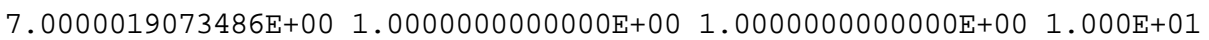
$\begin{array}{lll}7.3333353996277 \mathrm{E}+00 & 0.0000000000000 \mathrm{E}+00 & 0.0000000000000 \mathrm{E}+00 \quad 1.000 \mathrm{E}+01\end{array}$ $\begin{array}{llll}7.3333353996277 \mathrm{E}+00 & 0.0000000000000 \mathrm{E}+00 & 1.0000000000000 \mathrm{E}+00 & 1.000 \mathrm{E}+01\end{array}$ $7.3333353996277 \mathrm{E}+00 \quad 1.0000000000000 \mathrm{E}+00 \quad 0.0000000000000 \mathrm{E}+00 \quad 1.000 \mathrm{E}+01$ $7.3333353996277 \mathrm{E}+00 \quad 1.0000000000000 \mathrm{E}+00 \quad 1.0000000000000 \mathrm{E}+00 \quad 1.000 \mathrm{E}+01$ $\begin{array}{llll}7.6666688919067 \mathrm{E}+00 & 0.0000000000000 \mathrm{E}+00 & 0.0000000000000 \mathrm{E}+00 & 1.000 \mathrm{E}+01\end{array}$ $7.6666688919067 \mathrm{E}+00 \quad 0.0000000000000 \mathrm{E}+00 \quad 1.0000000000000 \mathrm{E}+00 \quad 1.000 \mathrm{E}+01$ $\begin{array}{llll}7.6666688919067 \mathrm{E}+00 & 1.0000000000000 \mathrm{E}+00 & 0.0000000000000 \mathrm{E}+00 & 1.000 \mathrm{E}+01\end{array}$ $7.6666688919067 \mathrm{E}+001.0000000000000 \mathrm{E}+00 \quad 1.0000000000000 \mathrm{E}+00 \quad 1.000 \mathrm{E}+01$ $8.0000028610229 \mathrm{E}+00 \quad 0.0000000000000 \mathrm{E}+00 \quad 0.0000000000000 \mathrm{E}+00 \quad 1.000 \mathrm{E}+01$ $8.0000028610229 \mathrm{E}+00 \quad 0.0000000000000 \mathrm{E}+00 \quad 1.0000000000000 \mathrm{E}+00 \quad 1.000 \mathrm{E}+01$ $8.0000028610229 \mathrm{E}+00 \quad 1.0000000000000 \mathrm{E}+00 \quad 0.0000000000000 \mathrm{E}+00 \quad 1.000 \mathrm{E}+01$ $8.0000028610229 \mathrm{E}+001.0000000000000 \mathrm{E}+00 \quad 1.0000000000000 \mathrm{E}+00 \quad 1.000 \mathrm{E}+01$ $8.3333358764648 \mathrm{E}+00 \quad 0.0000000000000 \mathrm{E}+00 \quad 0.0000000000000 \mathrm{E}+00 \quad 1.000 \mathrm{E}+01$ $8.3333358764648 \mathrm{E}+00 \quad 0.0000000000000 \mathrm{E}+00 \quad 1.0000000000000 \mathrm{E}+00 \quad 1.000 \mathrm{E}+01$ $8.3333358764648 \mathrm{E}+00 \quad 1.0000000000000 \mathrm{E}+00 \quad 0.0000000000000 \mathrm{E}+00 \quad 1.000 \mathrm{E}+01$ $8.3333358764648 \mathrm{E}+00 \quad 1.0000000000000 \mathrm{E}+00 \quad 1.0000000000000 \mathrm{E}+00 \quad 1.000 \mathrm{E}+01$ $8.6666698455811 \mathrm{E}+00 \quad 0.0000000000000 \mathrm{E}+00 \quad 0.0000000000000 \mathrm{E}+00 \quad 1.000 \mathrm{E}+01$ $8.6666698455811 \mathrm{E}+00 \quad 0.0000000000000 \mathrm{E}+00 \quad 1.0000000000000 \mathrm{E}+00 \quad 1.000 \mathrm{E}+01$ $8.6666698455811 \mathrm{E}+00 \quad 1.0000000000000 \mathrm{E}+00 \quad 0.0000000000000 \mathrm{E}+00 \quad 1.000 \mathrm{E}+01$ $8.6666698455811 \mathrm{E}+00 \quad 1.0000000000000 \mathrm{E}+00 \quad 1.0000000000000 \mathrm{E}+00 \quad 1.000 \mathrm{E}+01$ $9.0000038146973 \mathrm{E}+00 \quad 0.0000000000000 \mathrm{E}+00 \quad 0.0000000000000 \mathrm{E}+00 \quad 1.000 \mathrm{E}+01$ $9.0000038146973 \mathrm{E}+00 \quad 0.0000000000000 \mathrm{E}+00 \quad 1.0000000000000 \mathrm{E}+00 \quad 1.000 \mathrm{E}+01$ $9.0000038146973 \mathrm{E}+00 \quad 1.0000000000000 \mathrm{E}+00 \quad 0.0000000000000 \mathrm{E}+00 \quad 1.000 \mathrm{E}+01$ $9.0000038146973 \mathrm{E}+00 \quad 1.0000000000000 \mathrm{E}+00 \quad 1.0000000000000 \mathrm{E}+00 \quad 1.000 \mathrm{E}+01$ $9.3333368301392 \mathrm{E}+00 \quad 0.0000000000000 \mathrm{E}+00 \quad 0.0000000000000 \mathrm{E}+00 \quad 1.000 \mathrm{E}+01$ $9.3333368301392 \mathrm{E}+00 \quad 0.0000000000000 \mathrm{E}+00 \quad 1.0000000000000 \mathrm{E}+00 \quad 1.000 \mathrm{E}+01$ $9.3333368301392 \mathrm{E}+00 \quad 1.0000000000000 \mathrm{E}+00 \quad 0.0000000000000 \mathrm{E}+00 \quad 1.000 \mathrm{E}+01$ $9.3333368301392 \mathrm{E}+00 \quad 1.0000000000000 \mathrm{E}+00 \quad 1.0000000000000 \mathrm{E}+00 \quad 1.000 \mathrm{E}+01$ $9.6666707992554 \mathrm{E}+00 \quad 0.0000000000000 \mathrm{E}+00 \quad 0.0000000000000 \mathrm{E}+00 \quad 1.000 \mathrm{E}+01$ $9.6666707992554 \mathrm{E}+00 \quad 0.0000000000000 \mathrm{E}+00 \quad 1.0000000000000 \mathrm{E}+00 \quad 1.000 \mathrm{E}+01$ $9.6666707992554 \mathrm{E}+00 \quad 1.0000000000000 \mathrm{E}+00 \quad 0.0000000000000 \mathrm{E}+00 \quad 1.000 \mathrm{E}+01$ $9.6666707992554 \mathrm{E}+00 \quad 1.0000000000000 \mathrm{E}+00 \quad 1.0000000000000 \mathrm{E}+00 \quad 1.000 \mathrm{E}+01$ $1.0000000000000 \mathrm{E}+01 \quad 0.0000000000000 \mathrm{E}+00 \quad 0.0000000000000 \mathrm{E}+00 \quad 1.000 \mathrm{E}+01$ $1.0000000000000 \mathrm{E}+01 \quad 0.0000000000000 \mathrm{E}+00 \quad 1.0000000000000 \mathrm{E}+00 \quad 1.000 \mathrm{E}+01$ $1.0000000000000 \mathrm{E}+01 \quad 1.0000000000000 \mathrm{E}+00 \quad 0.0000000000000 \mathrm{E}+00 \quad 1.000 \mathrm{E}+01$ 


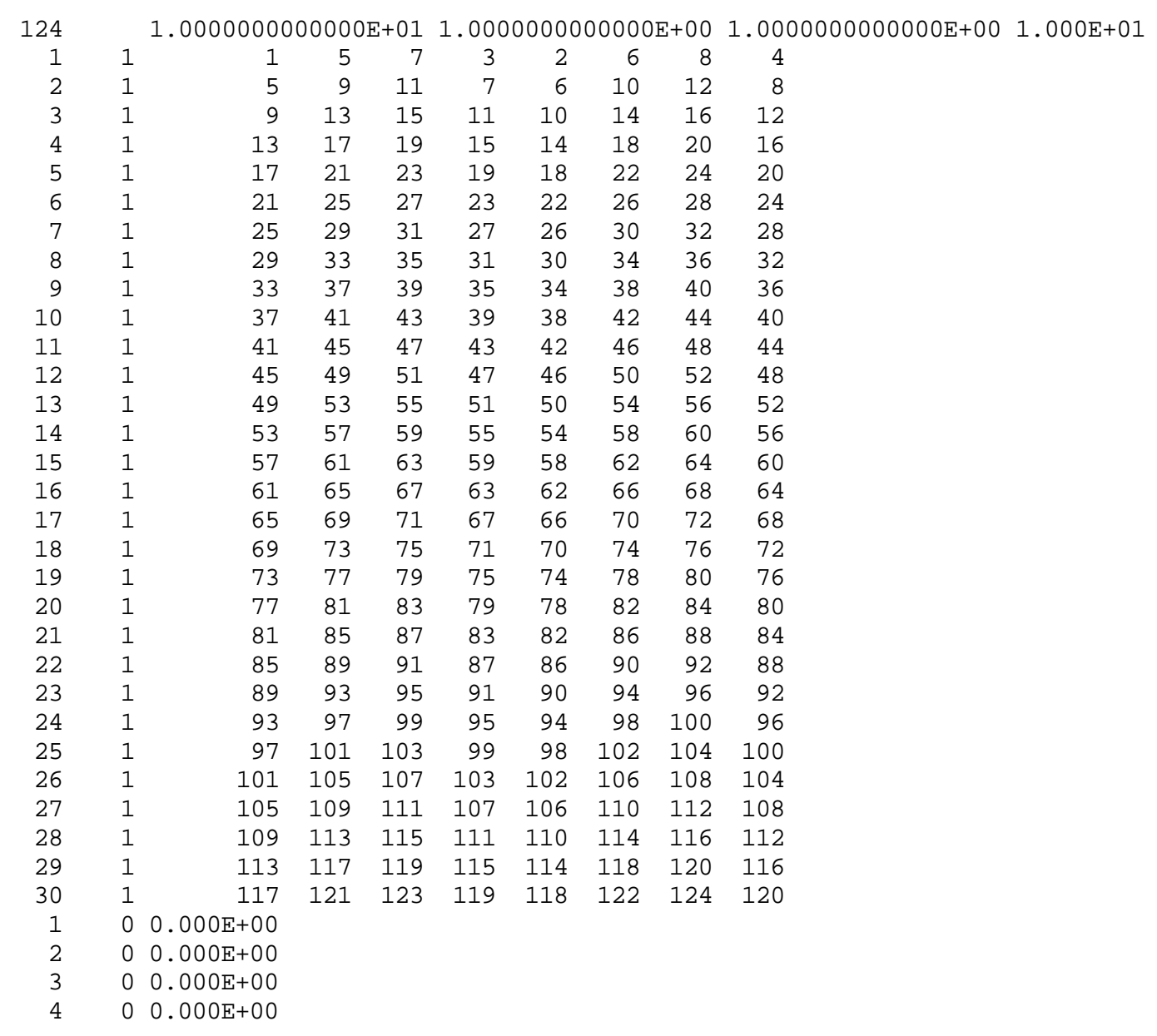

\subsection{Constant Flux Boundary Condition}

Problem: $\quad$ This case models the transient flow of heat in a semi-infinite solid with a flux boundary condition set at $\mathrm{x}=0$, a global initial temperature condition, and a set time interval.

Analytic solution: $\quad T_{x}=\frac{2 q_{s}^{\prime \prime} \sqrt{\alpha t} / \pi}{k} \times \exp \left(\frac{-x^{2}}{4 \alpha t}\right)-\frac{q_{s}^{\prime \prime} x}{k} \times \operatorname{erfc}\left(\frac{x}{2 \sqrt{\alpha t}}\right)+T_{i}$

(Incropera \& DeWitt, p.239)

Case parameters: $\quad$ Slab length - $\quad L=10$

Boundary conditions $-q_{s}^{\prime \prime}=10$ at $x=0$

Time - $\quad t=3$

$$
\begin{aligned}
& T_{i}=10 \\
& t=3
\end{aligned}
$$

Results: $\quad \underline{\text { x-coordinate }} \underline{\mathrm{T} \text { (analytical) }} \underline{\mathrm{T} \text { (TOPAZ3D) }}$

$0.00 \quad 29.54410 \quad 29.49939$ 


$\begin{array}{rrr}1.00 & 21.15053 & 21.11155 \\ 2.00 & 15.71963 & 15.69436 \\ 3.00 & 12.61184 & 12.60084 \\ 4.00 & 11.05296 & 11.05134 \\ 5.00 & 10.37218 & 10.37425 \\ 6.00 & 10.11469 & 10.11696 \\ 7.00 & 10.03067 & 10.03205 \\ 8.00 & 10.00709 & 10.00771 \\ 9.00 & 10.00142 & 10.00168 \\ 10.00 & 10.00024 & 10.00061\end{array}$

\section{Input file:}

C TOPAZ3D input file for transient linear flux boundary condition problem

$\begin{array}{rrrrrrrrrrrrrrr}1 & 124 & 30 & 0 & & 0 & 0 & 0 & 0 & 0 & 1 & 0 & 0 & 0 & 0 \\ 0 & 0 & 0 & 1 & 0 & 0 & 0 & 0 & 0 & 0 & 0 & 0 & 0 & \end{array}$

$\begin{array}{lllllll}1 & 0 & 0 & 0 & 0 & 0.000 \mathrm{E}+00\end{array}$

$\begin{array}{llllllll}0.000 \mathrm{E}+00 & 3.000 \mathrm{E}+00 & 1.000 \mathrm{E}-01 & 0.000 \mathrm{E}+00 & 0.000 \mathrm{E}+00 & 0.000 \mathrm{E}+00 & 0.000 \mathrm{E}+00\end{array}$

$\begin{array}{lllllll}0 & 0 & 0 & 0 & 0 & 0.000 \mathrm{E}+00 & 0.000 \mathrm{E}+00\end{array}$

$\begin{array}{llllllll}1 & 1 & 1.000 \mathrm{E}+00 & 0.000 \mathrm{E}+00 & 0.000 \mathrm{E}+00 & 0 & 0.000 \mathrm{E}+00 & 0\end{array}$

Material Type 1 - Isotropic

$1.000 \mathrm{E}+001.000 \mathrm{E}+00$

$10.0000000000000 \mathrm{E}+00 \quad 0.0000000000000 \mathrm{E}+00 \quad 0.0000000000000 \mathrm{E}+00 \quad 1.000 \mathrm{E}+01$

$2 \quad 0.0000000000000 \mathrm{E}+00 \quad 0.0000000000000 \mathrm{E}+00 \quad 1.0000000000000 \mathrm{E}+00 \quad 1.000 \mathrm{E}+01$

$3 \quad 0.0000000000000 \mathrm{E}+00 \quad 1.0000000000000 \mathrm{E}+00 \quad 0.0000000000000 \mathrm{E}+00 \quad 1.000 \mathrm{E}+01$

$4 \quad 0.0000000000000 \mathrm{E}+00 \quad 1.0000000000000 \mathrm{E}+00 \quad 1.0000000000000 \mathrm{E}+001.000 \mathrm{E}+01$

$5 \quad 3.3333334326744 \mathrm{E}-01 \quad 0.0000000000000 \mathrm{E}+00 \quad 0.0000000000000 \mathrm{E}+00 \quad 1.000 \mathrm{E}+01$

$6 \quad 3.3333334326744 \mathrm{E}-01 \quad 0.0000000000000 \mathrm{E}+00 \quad 1.0000000000000 \mathrm{E}+00 \quad 1.000 \mathrm{E}+01$

$7 \quad 3.3333334326744 \mathrm{E}-01 \quad 1.0000000000000 \mathrm{E}+00 \quad 0.0000000000000 \mathrm{E}+00 \quad 1.000 \mathrm{E}+01$

$8 \quad 3.3333334326744 \mathrm{E}-01 \quad 1.0000000000000 \mathrm{E}+00 \quad 1.0000000000000 \mathrm{E}+001.000 \mathrm{E}+01$

$96.6666668653488 \mathrm{E}-01 \quad 0.0000000000000 \mathrm{E}+00 \quad 0.0000000000000 \mathrm{E}+00 \quad 1.000 \mathrm{E}+01$

$106.6666668653488 \mathrm{E}-01 \quad 0.0000000000000 \mathrm{E}+00 \quad 1.0000000000000 \mathrm{E}+00 \quad 1.000 \mathrm{E}+01$

$116.6666668653488 \mathrm{E}-01 \quad 1.0000000000000 \mathrm{E}+00 \quad 0.0000000000000 \mathrm{E}+00 \quad 1.000 \mathrm{E}+01$

$126.6666668653488 \mathrm{E}-01 \quad 1.0000000000000 \mathrm{E}+00 \quad 1.0000000000000 \mathrm{E}+001.000 \mathrm{E}+01$

$131.0000001192093 \mathrm{E}+00 \quad 0.0000000000000 \mathrm{E}+00 \quad 0.0000000000000 \mathrm{E}+001.000 \mathrm{E}+01$

$141.0000001192093 \mathrm{E}+00 \quad 0.0000000000000 \mathrm{E}+00 \quad 1.0000000000000 \mathrm{E}+001.000 \mathrm{E}+01$

$151.0000001192093 \mathrm{E}+00 \quad 1.0000000000000 \mathrm{E}+00 \quad 0.0000000000000 \mathrm{E}+001.000 \mathrm{E}+01$

$161.0000001192093 \mathrm{E}+00 \quad 1.0000000000000 \mathrm{E}+00 \quad 1.0000000000000 \mathrm{E}+001.000 \mathrm{E}+01$

$171.3333333730698 \mathrm{E}+00 \quad 0.0000000000000 \mathrm{E}+00 \quad 0.0000000000000 \mathrm{E}+00 \quad 1.000 \mathrm{E}+01$

$181.3333333730698 \mathrm{E}+00 \quad 0.0000000000000 \mathrm{E}+00 \quad 1.0000000000000 \mathrm{E}+00 \quad 1.000 \mathrm{E}+01$

$191.3333333730698 \mathrm{E}+00 \quad 1.0000000000000 \mathrm{E}+00 \quad 0.0000000000000 \mathrm{E}+00 \quad 1.000 \mathrm{E}+01$

$201.3333333730698 \mathrm{E}+001.0000000000000 \mathrm{E}+00 \quad 1.0000000000000 \mathrm{E}+001.000 \mathrm{E}+01$

$211.6666667461395 \mathrm{E}+00 \quad 0.0000000000000 \mathrm{E}+00 \quad 0.0000000000000 \mathrm{E}+00 \quad 1.000 \mathrm{E}+01$

$221.6666667461395 \mathrm{E}+00 \quad 0.0000000000000 \mathrm{E}+00 \quad 1.0000000000000 \mathrm{E}+00 \quad 1.000 \mathrm{E}+01$

$1.6666667461395 \mathrm{E}+00 \quad 1.0000000000000 \mathrm{E}+00 \quad 0.0000000000000 \mathrm{E}+00 \quad 1.000 \mathrm{E}+01$

$1.6666667461395 \mathrm{E}+00 \quad 1.0000000000000 \mathrm{E}+00 \quad 1.0000000000000 \mathrm{E}+00 \quad 1.000 \mathrm{E}+01$

$2.0000000000000 \mathrm{E}+00 \quad 0.0000000000000 \mathrm{E}+00 \quad 0.0000000000000 \mathrm{E}+00 \quad 1.000 \mathrm{E}+01$ $2.0000000000000 \mathrm{E}+00 \quad 0.0000000000000 \mathrm{E}+00 \quad 1.0000000000000 \mathrm{E}+00 \quad 1.000 \mathrm{E}+01$ $2.0000000000000 \mathrm{E}+00 \quad 1.0000000000000 \mathrm{E}+00 \quad 0.0000000000000 \mathrm{E}+00 \quad 1.000 \mathrm{E}+01$ $2.0000000000000 \mathrm{E}+001.0000000000000 \mathrm{E}+00 \quad 1.0000000000000 \mathrm{E}+00 \quad 1.000 \mathrm{E}+01$ $2.3333332538605 \mathrm{E}+00 \quad 0.0000000000000 \mathrm{E}+00 \quad 0.0000000000000 \mathrm{E}+00 \quad 1.000 \mathrm{E}+01$ $2.3333332538605 \mathrm{E}+00 \quad 0.0000000000000 \mathrm{E}+00 \quad 1.0000000000000 \mathrm{E}+00 \quad 1.000 \mathrm{E}+01$ $2.3333332538605 \mathrm{E}+00 \quad 1.0000000000000 \mathrm{E}+00 \quad 0.0000000000000 \mathrm{E}+00 \quad 1.000 \mathrm{E}+01$ $2.3333332538605 \mathrm{E}+00 \quad 1.0000000000000 \mathrm{E}+00 \quad 1.0000000000000 \mathrm{E}+00 \quad 1.000 \mathrm{E}+01$ $2.6666667461395 \mathrm{E}+00 \quad 0.0000000000000 \mathrm{E}+00 \quad 0.0000000000000 \mathrm{E}+00 \quad 1.000 \mathrm{E}+01$ $2.6666667461395 \mathrm{E}+00 \quad 0.0000000000000 \mathrm{E}+00 \quad 1.0000000000000 \mathrm{E}+00 \quad 1.000 \mathrm{E}+01$ $2.6666667461395 \mathrm{E}+00 \quad 1.0000000000000 \mathrm{E}+00 \quad 0.0000000000000 \mathrm{E}+00 \quad 1.000 \mathrm{E}+01$ $2.6666667461395 \mathrm{E}+00 \quad 1.0000000000000 \mathrm{E}+00 \quad 1.0000000000000 \mathrm{E}+00 \quad 1.000 \mathrm{E}+01$ $3.0000000000000 \mathrm{E}+00 \quad 0.0000000000000 \mathrm{E}+00 \quad 0.0000000000000 \mathrm{E}+00 \quad 1.000 \mathrm{E}+01$ $3.0000000000000 \mathrm{E}+00 \quad 0.0000000000000 \mathrm{E}+00 \quad 1.0000000000000 \mathrm{E}+00 \quad 1.000 \mathrm{E}+01$ 
$3.0000000000000 \mathrm{E}+00 \quad 1.0000000000000 \mathrm{E}+00 \quad 0.0000000000000 \mathrm{E}+00 \quad 1.000 \mathrm{E}+01$ 3.0000000000000E+00 1.0000000000000E+00 1.0000000000000E+00 1.000E+01 $\begin{array}{llll}3.3333334922791 \mathrm{E}+00 & 0.0000000000000 \mathrm{E}+00 & 0.0000000000000 \mathrm{E}+00 & 1.000 \mathrm{E}+01\end{array}$ $3.3333334922791 \mathrm{E}+00 \quad 0.0000000000000 \mathrm{E}+00 \quad 1.0000000000000 \mathrm{E}+00 \quad 1.000 \mathrm{E}+01$ $3.3333334922791 \mathrm{E}+00 \quad 1.0000000000000 \mathrm{E}+00 \quad 0.0000000000000 \mathrm{E}+00 \quad 1.000 \mathrm{E}+01$ $3.3333334922791 \mathrm{E}+00 \quad 1.0000000000000 \mathrm{E}+00 \quad 1.0000000000000 \mathrm{E}+00 \quad 1.000 \mathrm{E}+01$ $3.6666667461395 \mathrm{E}+00 \quad 0.0000000000000 \mathrm{E}+00 \quad 0.0000000000000 \mathrm{E}+00 \quad 1.000 \mathrm{E}+01$ $3.6666667461395 \mathrm{E}+00 \quad 0.0000000000000 \mathrm{E}+00 \quad 1.0000000000000 \mathrm{E}+00 \quad 1.000 \mathrm{E}+01$ $3.6666667461395 \mathrm{E}+00 \quad 1.0000000000000 \mathrm{E}+00 \quad 0.0000000000000 \mathrm{E}+00 \quad 1.000 \mathrm{E}+01$ $3.6666667461395 \mathrm{E}+00 \quad 1.0000000000000 \mathrm{E}+00 \quad 1.0000000000000 \mathrm{E}+00 \quad 1.000 \mathrm{E}+01$ $4.0000000000000 \mathrm{E}+00 \quad 0.0000000000000 \mathrm{E}+00 \quad 0.0000000000000 \mathrm{E}+00 \quad 1.000 \mathrm{E}+01$ $4.0000000000000 \mathrm{E}+00 \quad 0.0000000000000 \mathrm{E}+00 \quad 1.0000000000000 \mathrm{E}+00 \quad 1.000 \mathrm{E}+01$ $4.0000000000000 \mathrm{E}+00 \quad 1.0000000000000 \mathrm{E}+00 \quad 0.0000000000000 \mathrm{E}+00 \quad 1.000 \mathrm{E}+01$ $4.0000000000000 \mathrm{E}+001.0000000000000 \mathrm{E}+00 \quad 1.0000000000000 \mathrm{E}+00 \quad 1.000 \mathrm{E}+01$ $\begin{array}{lll}4.3333334922791 \mathrm{E}+00 & 0.0000000000000 \mathrm{E}+00 & 0.0000000000000 \mathrm{E}+00 \quad 1.000 \mathrm{E}+01\end{array}$ $\begin{array}{llll}4.3333334922791 \mathrm{E}+00 & 0.0000000000000 \mathrm{E}+00 & 1.0000000000000 \mathrm{E}+00 \quad 1.000 \mathrm{E}+01\end{array}$ $4.3333334922791 \mathrm{E}+001.0000000000000 \mathrm{E}+00 \quad 0.0000000000000 \mathrm{E}+00 \quad 1.000 \mathrm{E}+01$ $4.3333334922791 \mathrm{E}+001.0000000000000 \mathrm{E}+00 \quad 1.0000000000000 \mathrm{E}+00 \quad 1.000 \mathrm{E}+01$ $\begin{array}{lllll}4.6666665077209 \mathrm{E}+00 & 0.0000000000000 \mathrm{E}+00 & 0.0000000000000 \mathrm{E}+00 & 1.000 \mathrm{E}+01\end{array}$ $\begin{array}{lll}4.6666665077209 \mathrm{E}+00 & 0.0000000000000 \mathrm{E}+00 & 1.0000000000000 \mathrm{E}+00 \quad 1.000 \mathrm{E}+01\end{array}$ $\begin{array}{llll}4.6666665077209 \mathrm{E}+00 & 1.0000000000000 \mathrm{E}+00 & 0.0000000000000 \mathrm{E}+00 & 1.000 \mathrm{E}+01\end{array}$ $4.6666665077209 \mathrm{E}+00 \quad 1.0000000000000 \mathrm{E}+00 \quad 1.0000000000000 \mathrm{E}+00 \quad 1.000 \mathrm{E}+01$ $5.0000000000000 \mathrm{E}+00 \quad 0.0000000000000 \mathrm{E}+00 \quad 0.0000000000000 \mathrm{E}+00 \quad 1.000 \mathrm{E}+01$ $5.0000000000000 \mathrm{E}+00 \quad 0.0000000000000 \mathrm{E}+00 \quad 1.0000000000000 \mathrm{E}+00 \quad 1.000 \mathrm{E}+01$ $5.0000000000000 \mathrm{E}+00 \quad 1.0000000000000 \mathrm{E}+00 \quad 0.0000000000000 \mathrm{E}+00 \quad 1.000 \mathrm{E}+01$ $5.0000000000000 \mathrm{E}+001.0000000000000 \mathrm{E}+00 \quad 1.0000000000000 \mathrm{E}+00 \quad 1.000 \mathrm{E}+01$ $\begin{array}{llll}5.3333334922791 \mathrm{E}+00 & 0.0000000000000 \mathrm{E}+00 & 0.0000000000000 \mathrm{E}+00 & 1.000 \mathrm{E}+01\end{array}$ $\begin{array}{llll}5.3333334922791 \mathrm{E}+00 & 0.0000000000000 \mathrm{E}+00 & 1.0000000000000 \mathrm{E}+00 & 1.000 \mathrm{E}+01\end{array}$ $\begin{array}{llll}5.3333334922791 \mathrm{E}+00 & 1.0000000000000 \mathrm{E}+00 & 0.0000000000000 \mathrm{E}+00 & 1.000 \mathrm{E}+01\end{array}$ $5.3333334922791 \mathrm{E}+001.0000000000000 \mathrm{E}+00 \quad 1.0000000000000 \mathrm{E}+00 \quad 1.000 \mathrm{E}+01$ $\begin{array}{llll}5.6666669845581 \mathrm{E}+00 & 0.0000000000000 \mathrm{E}+00 & 0.0000000000000 \mathrm{E}+00 & 1.000 \mathrm{E}+01\end{array}$ $\begin{array}{llll}5.6666669845581 \mathrm{E}+00 & 0.0000000000000 \mathrm{E}+00 & 1.0000000000000 \mathrm{E}+00 \quad 1.000 \mathrm{E}+01\end{array}$ $5.6666669845581 \mathrm{E}+00 \quad 1.0000000000000 \mathrm{E}+00 \quad 0.0000000000000 \mathrm{E}+00 \quad 1.000 \mathrm{E}+01$ $5.6666669845581 \mathrm{E}+00 \quad 1.0000000000000 \mathrm{E}+00 \quad 1.0000000000000 \mathrm{E}+00 \quad 1.000 \mathrm{E}+01$ $6.0000009536743 \mathrm{E}+00 \quad 0.0000000000000 \mathrm{E}+00 \quad 0.0000000000000 \mathrm{E}+00 \quad 1.000 \mathrm{E}+01$ $6.0000009536743 \mathrm{E}+00 \quad 0.0000000000000 \mathrm{E}+00 \quad 1.0000000000000 \mathrm{E}+00 \quad 1.000 \mathrm{E}+01$ $6.0000009536743 \mathrm{E}+00 \quad 1.0000000000000 \mathrm{E}+00 \quad 0.0000000000000 \mathrm{E}+00 \quad 1.000 \mathrm{E}+01$ $6.0000009536743 \mathrm{E}+00 \quad 1.0000000000000 \mathrm{E}+00 \quad 1.0000000000000 \mathrm{E}+00 \quad 1.000 \mathrm{E}+01$ $6.3333344459534 \mathrm{E}+00 \quad 0.0000000000000 \mathrm{E}+00 \quad 0.0000000000000 \mathrm{E}+00 \quad 1.000 \mathrm{E}+01$ $6.3333344459534 \mathrm{E}+00 \quad 0.0000000000000 \mathrm{E}+00 \quad 1.0000000000000 \mathrm{E}+00 \quad 1.000 \mathrm{E}+01$ $6.3333344459534 \mathrm{E}+00 \quad 1.0000000000000 \mathrm{E}+00 \quad 0.0000000000000 \mathrm{E}+00 \quad 1.000 \mathrm{E}+01$ $6.3333344459534 \mathrm{E}+00 \quad 1.0000000000000 \mathrm{E}+00 \quad 1.0000000000000 \mathrm{E}+00 \quad 1.000 \mathrm{E}+01$ $\begin{array}{llll}6.6666679382324 \mathrm{E}+00 & 0.0000000000000 \mathrm{E}+00 & 0.0000000000000 \mathrm{E}+00 & 1.000 \mathrm{E}+01\end{array}$ $\begin{array}{llll}6.6666679382324 \mathrm{E}+00 & 0.0000000000000 \mathrm{E}+00 & 1.0000000000000 \mathrm{E}+00 & 1.000 \mathrm{E}+01\end{array}$ $6.6666679382324 \mathrm{E}+00 \quad 1.0000000000000 \mathrm{E}+00 \quad 0.0000000000000 \mathrm{E}+00 \quad 1.000 \mathrm{E}+01$ $6.6666679382324 \mathrm{E}+00 \quad 1.0000000000000 \mathrm{E}+00 \quad 1.0000000000000 \mathrm{E}+00 \quad 1.000 \mathrm{E}+01$ $7.0000019073486 \mathrm{E}+00 \quad 0.0000000000000 \mathrm{E}+00 \quad 0.0000000000000 \mathrm{E}+00 \quad 1.000 \mathrm{E}+01$ $7.0000019073486 \mathrm{E}+00 \quad 0.0000000000000 \mathrm{E}+00 \quad 1.0000000000000 \mathrm{E}+00 \quad 1.000 \mathrm{E}+01$ $\begin{array}{llll}7.0000019073486 \mathrm{E}+00 & 1.0000000000000 \mathrm{E}+00 & 0.0000000000000 \mathrm{E}+00 & 1.000 \mathrm{E}+01\end{array}$ $7.0000019073486 \mathrm{E}+001.0000000000000 \mathrm{E}+00 \quad 1.0000000000000 \mathrm{E}+00 \quad 1.000 \mathrm{E}+01$ $\begin{array}{llll}7.3333353996277 \mathrm{E}+00 & 0.0000000000000 \mathrm{E}+00 & 0.0000000000000 \mathrm{E}+00 & 1.000 \mathrm{E}+01\end{array}$ $\begin{array}{llll}7.3333353996277 \mathrm{E}+00 & 0.0000000000000 \mathrm{E}+00 & 1.0000000000000 \mathrm{E}+00 & 1.000 \mathrm{E}+01\end{array}$ $\begin{array}{llll}7.3333353996277 \mathrm{E}+00 & 1.0000000000000 \mathrm{E}+00 & 0.0000000000000 \mathrm{E}+00 & 1.000 \mathrm{E}+01\end{array}$ $\begin{array}{llll}7.3333353996277 \mathrm{E}+00 & 1.0000000000000 \mathrm{E}+00 & 1.0000000000000 \mathrm{E}+00 & 1.000 \mathrm{E}+01\end{array}$ $\begin{array}{llll}7.6666688919067 \mathrm{E}+00 & 0.0000000000000 \mathrm{E}+00 & 0.0000000000000 \mathrm{E}+00 \quad 1.000 \mathrm{E}+01\end{array}$ $\begin{array}{llll}7.6666688919067 \mathrm{E}+00 & 0.0000000000000 \mathrm{E}+00 & 1.0000000000000 \mathrm{E}+00 & 1.000 \mathrm{E}+01\end{array}$ $7.6666688919067 \mathrm{E}+00 \quad 1.0000000000000 \mathrm{E}+00 \quad 0.0000000000000 \mathrm{E}+00 \quad 1.000 \mathrm{E}+01$ $7.6666688919067 \mathrm{E}+00 \quad 1.0000000000000 \mathrm{E}+00 \quad 1.0000000000000 \mathrm{E}+00 \quad 1.000 \mathrm{E}+01$ $8.0000028610229 \mathrm{E}+00 \quad 0.0000000000000 \mathrm{E}+00 \quad 0.0000000000000 \mathrm{E}+00 \quad 1.000 \mathrm{E}+01$ $8.0000028610229 \mathrm{E}+00 \quad 0.0000000000000 \mathrm{E}+00 \quad 1.0000000000000 \mathrm{E}+00 \quad 1.000 \mathrm{E}+01$ $8.0000028610229 \mathrm{E}+00 \quad 1.0000000000000 \mathrm{E}+00 \quad 0.0000000000000 \mathrm{E}+00 \quad 1.000 \mathrm{E}+01$ $8.0000028610229 \mathrm{E}+001.0000000000000 \mathrm{E}+00 \quad 1.0000000000000 \mathrm{E}+00 \quad 1.000 \mathrm{E}+01$ $8.3333358764648 \mathrm{E}+00 \quad 0.0000000000000 \mathrm{E}+00 \quad 0.0000000000000 \mathrm{E}+00 \quad 1.000 \mathrm{E}+01$ $8.3333358764648 \mathrm{E}+00 \quad 0.0000000000000 \mathrm{E}+00 \quad 1.0000000000000 \mathrm{E}+00 \quad 1.000 \mathrm{E}+01$ 


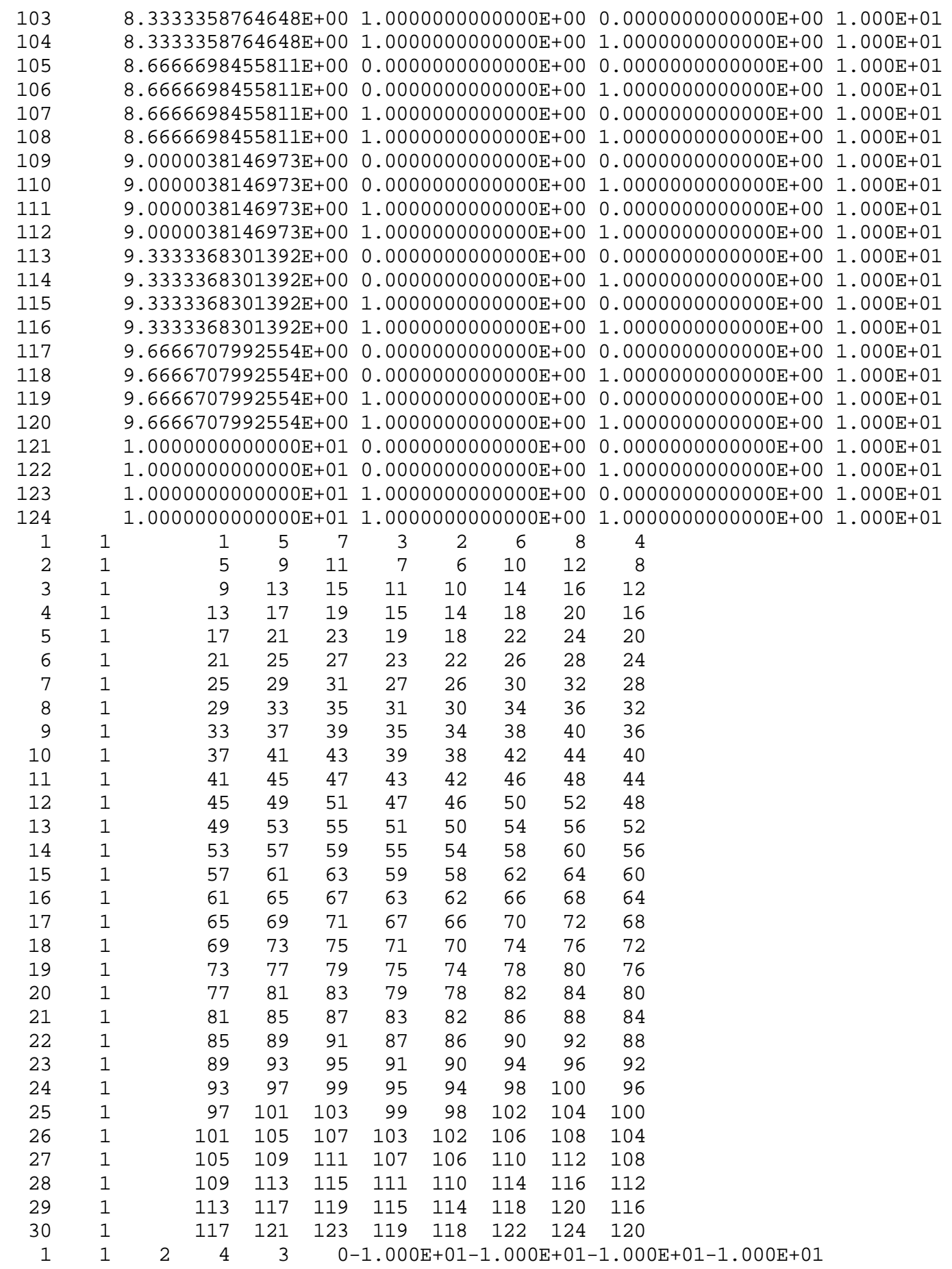

3.3 Constant Convection Boundary Condition

Problem:

This case models the transient flow of heat in a semi-infinite solid with a convection boundary condition set at $\mathrm{x}=0$, a global initial temperature condition, and a set time interval. 
Analytic solution:

$$
T_{x}=\left(T_{\infty}-T_{i}\right)\left[\operatorname{erfc}\left(\frac{x}{2 \sqrt{\alpha t}}\right)-\left[\exp \left(\frac{h x}{k}+\frac{h^{2} \alpha t}{k^{2}}\right)\right]\left[\operatorname{erfc}\left(\frac{x}{2 \sqrt{\alpha t}}+\frac{h \sqrt{\alpha t}}{k}\right)\right]\right]+T_{i}
$$

(Incropera \& DeWitt, p.239)

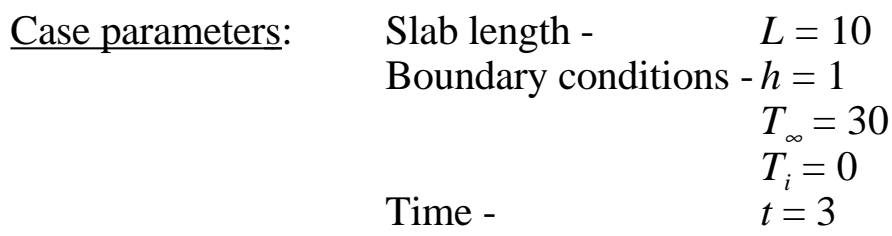

Results:

$\underline{\mathrm{x} \text {-coordinate }} \underline{\mathrm{T} \text { (analytical) }} \underline{\mathrm{T}(\mathrm{TOPAZ3D})}$

$\begin{array}{rrr}0.00 & 21.37976 & 21.38841 \\ 1.00 & 13.50406 & 13.50239 \\ 2.00 & 7.56971 & 7.56577 \\ 3.00 & 3.73315 & 3.73301 \\ 4.00 & 1.60733 & 1.61284 \\ 5.00 & 0.60249 & 0.60799 \\ 6.00 & 0.19735 & 0.19963 \\ 7.00 & 0.04923 & 0.05708 \\ 8.00 & 0.03272 & 0.01425 \\ 9.00 & 0.00716 & 0.00320 \\ 10.00 & 0.00134 & 0.00119\end{array}$

Input file:

c TOPAZ3D input file for transient linear convection boundary condition problem

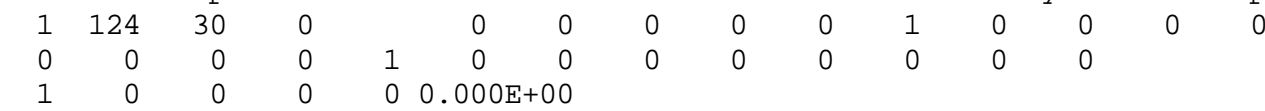

$\begin{array}{lllllllll}0.000 \mathrm{E}+00 & 3.000 \mathrm{E}+00 & 1.000 \mathrm{E}-01 & 0.000 \mathrm{E}+00 & 0.000 \mathrm{E}+00 & 0.000 \mathrm{E}+00 & 0.000 \mathrm{E}+00\end{array}$

$\begin{array}{lllllll}0 & 0 & 0 & 0 & 0 & 0.000 \mathrm{E}+00 & 0.000 \mathrm{E}+00\end{array}$

$\begin{array}{llllllll}1 & 1 & 1.000 \mathrm{E}+00 & 0.000 \mathrm{E}+00 & 0.000 \mathrm{E}+00 & 0 & 0.000 \mathrm{E}+00 & 0\end{array}$

Material Type 1 - Isotropic

1.000E+00 1.000E+00

$10.0000000000000 \mathrm{E}+00 \quad 0.0000000000000 \mathrm{E}+00 \quad 0.0000000000000 \mathrm{E}+00 \quad 0.000 \mathrm{E}+00$

$2 \quad 0.0000000000000 \mathrm{E}+00 \quad 0.0000000000000 \mathrm{E}+00 \quad 1.0000000000000 \mathrm{E}+00 \quad 0.000 \mathrm{E}+00$

$30.0000000000000 \mathrm{E}+001.0000000000000 \mathrm{E}+00 \quad 0.0000000000000 \mathrm{E}+00 \quad 0.000 \mathrm{E}+00$

$4 \quad 0.0000000000000 \mathrm{E}+001.0000000000000 \mathrm{E}+001.0000000000000 \mathrm{E}+00 \quad 0.000 \mathrm{E}+00$

$53.3333334326744 \mathrm{E}-01 \quad 0.0000000000000 \mathrm{E}+00 \quad 0.0000000000000 \mathrm{E}+00 \quad 0.000 \mathrm{E}+00$

$6 \quad 3.3333334326744 \mathrm{E}-01 \quad 0.0000000000000 \mathrm{E}+00 \quad 1.0000000000000 \mathrm{E}+00 \quad 0.000 \mathrm{E}+00$

$7 \quad 3.3333334326744 \mathrm{E}-01 \quad 1.0000000000000 \mathrm{E}+00 \quad 0.0000000000000 \mathrm{E}+00 \quad 0.000 \mathrm{E}+00$

$83.3333334326744 \mathrm{E}-01$ 1.0000000000000E+00 1.0000000000000E+00 0.000E+00

$96.6666668653488 \mathrm{E}-01 \quad 0.0000000000000 \mathrm{E}+00 \quad 0.0000000000000 \mathrm{E}+00 \quad 0.000 \mathrm{E}+00$

$106.6666668653488 \mathrm{E}-01 \quad 0.0000000000000 \mathrm{E}+00 \quad 1.0000000000000 \mathrm{E}+00 \quad 0.000 \mathrm{E}+00$

$116.6666668653488 \mathrm{E}-01 \quad 1.0000000000000 \mathrm{E}+00 \quad 0.0000000000000 \mathrm{E}+00 \quad 0.000 \mathrm{E}+00$

$126.6666668653488 \mathrm{E}-01 \quad 1.0000000000000 \mathrm{E}+00 \quad 1.0000000000000 \mathrm{E}+00 \quad 0.000 \mathrm{E}+00$

$1.0000001192093 \mathrm{E}+00 \quad 0.0000000000000 \mathrm{E}+00 \quad 0.0000000000000 \mathrm{E}+00 \quad 0.000 \mathrm{E}+00$

$1.0000001192093 \mathrm{E}+00 \quad 0.0000000000000 \mathrm{E}+00 \quad 1.0000000000000 \mathrm{E}+00 \quad 0.000 \mathrm{E}+00$

$1.0000001192093 \mathrm{E}+00 \quad 1.0000000000000 \mathrm{E}+00 \quad 0.0000000000000 \mathrm{E}+00 \quad 0.000 \mathrm{E}+00$

$1.0000001192093 \mathrm{E}+001.0000000000000 \mathrm{E}+00 \quad 1.0000000000000 \mathrm{E}+00 \quad 0.000 \mathrm{E}+00$

$1.3333333730698 \mathrm{E}+00 \quad 0.0000000000000 \mathrm{E}+00 \quad 0.0000000000000 \mathrm{E}+00 \quad 0.000 \mathrm{E}+00$

$1.3333333730698 \mathrm{E}+00 \quad 0.0000000000000 \mathrm{E}+00 \quad 1.0000000000000 \mathrm{E}+00 \quad 0.000 \mathrm{E}+00$ 
$1.3333333730698 \mathrm{E}+00 \quad 1.0000000000000 \mathrm{E}+00 \quad 0.0000000000000 \mathrm{E}+00 \quad 0.000 \mathrm{E}+00$ $1.3333333730698 \mathrm{E}+00 \quad 1.0000000000000 \mathrm{E}+00 \quad 1.0000000000000 \mathrm{E}+00 \quad 0.000 \mathrm{E}+00$ $\begin{array}{lllll}1.6666667461395 \mathrm{E}+00 & 0.0000000000000 \mathrm{E}+00 & 0.0000000000000 \mathrm{E}+00 & 0.000 \mathrm{E}+00\end{array}$ $\begin{array}{llll}1.6666667461395 \mathrm{E}+00 & 0.0000000000000 \mathrm{E}+00 & 1.0000000000000 \mathrm{E}+00 \quad 0.000 \mathrm{E}+00\end{array}$ $1.6666667461395 \mathrm{E}+00 \quad 1.0000000000000 \mathrm{E}+00 \quad 0.0000000000000 \mathrm{E}+00 \quad 0.000 \mathrm{E}+00$ $1.6666667461395 \mathrm{E}+001.0000000000000 \mathrm{E}+00 \quad 1.0000000000000 \mathrm{E}+00 \quad 0.000 \mathrm{E}+00$ $2.0000000000000 \mathrm{E}+00 \quad 0.0000000000000 \mathrm{E}+00 \quad 0.0000000000000 \mathrm{E}+00 \quad 0.000 \mathrm{E}+00$ $2.0000000000000 \mathrm{E}+00 \quad 0.0000000000000 \mathrm{E}+00 \quad 1.0000000000000 \mathrm{E}+00 \quad 0.000 \mathrm{E}+00$ $2.0000000000000 \mathrm{E}+00 \quad 1.0000000000000 \mathrm{E}+00 \quad 0.0000000000000 \mathrm{E}+00 \quad 0.000 \mathrm{E}+00$ $2.0000000000000 \mathrm{E}+001.0000000000000 \mathrm{E}+00 \quad 1.0000000000000 \mathrm{E}+00 \quad 0.000 \mathrm{E}+00$ $2.3333332538605 \mathrm{E}+00 \quad 0.0000000000000 \mathrm{E}+00 \quad 0.0000000000000 \mathrm{E}+00 \quad 0.000 \mathrm{E}+00$ $2.3333332538605 \mathrm{E}+00 \quad 0.0000000000000 \mathrm{E}+00 \quad 1.0000000000000 \mathrm{E}+00 \quad 0.000 \mathrm{E}+00$ $2.3333332538605 \mathrm{E}+00 \quad 1.0000000000000 \mathrm{E}+00 \quad 0.0000000000000 \mathrm{E}+00 \quad 0.000 \mathrm{E}+00$ $2.3333332538605 \mathrm{E}+00 \quad 1.0000000000000 \mathrm{E}+00 \quad 1.0000000000000 \mathrm{E}+00 \quad 0.000 \mathrm{E}+00$ $2.6666667461395 \mathrm{E}+00 \quad 0.0000000000000 \mathrm{E}+00 \quad 0.0000000000000 \mathrm{E}+00 \quad 0.000 \mathrm{E}+00$ $2.6666667461395 \mathrm{E}+00 \quad 0.0000000000000 \mathrm{E}+00 \quad 1.0000000000000 \mathrm{E}+00 \quad 0.000 \mathrm{E}+00$ $2.6666667461395 \mathrm{E}+00 \quad 1.0000000000000 \mathrm{E}+00 \quad 0.0000000000000 \mathrm{E}+00 \quad 0.000 \mathrm{E}+00$ $2.6666667461395 \mathrm{E}+001.0000000000000 \mathrm{E}+00 \quad 1.0000000000000 \mathrm{E}+00 \quad 0.000 \mathrm{E}+00$ $3.0000000000000 \mathrm{E}+00 \quad 0.0000000000000 \mathrm{E}+00 \quad 0.0000000000000 \mathrm{E}+00 \quad 0.000 \mathrm{E}+00$ $3.0000000000000 \mathrm{E}+00 \quad 0.0000000000000 \mathrm{E}+00 \quad 1.0000000000000 \mathrm{E}+00 \quad 0.000 \mathrm{E}+00$ $3.0000000000000 \mathrm{E}+00 \quad 1.0000000000000 \mathrm{E}+00 \quad 0.0000000000000 \mathrm{E}+00 \quad 0.000 \mathrm{E}+00$ $3.0000000000000 \mathrm{E}+001.0000000000000 \mathrm{E}+00 \quad 1.0000000000000 \mathrm{E}+00 \quad 0.000 \mathrm{E}+00$ $3.3333334922791 \mathrm{E}+00 \quad 0.0000000000000 \mathrm{E}+00 \quad 0.0000000000000 \mathrm{E}+00 \quad 0.000 \mathrm{E}+00$ $3.3333334922791 \mathrm{E}+00 \quad 0.0000000000000 \mathrm{E}+00 \quad 1.0000000000000 \mathrm{E}+00 \quad 0.000 \mathrm{E}+00$ $3.3333334922791 \mathrm{E}+00 \quad 1.0000000000000 \mathrm{E}+00 \quad 0.0000000000000 \mathrm{E}+00 \quad 0.000 \mathrm{E}+00$ $3.3333334922791 \mathrm{E}+00 \quad 1.0000000000000 \mathrm{E}+00 \quad 1.0000000000000 \mathrm{E}+00 \quad 0.000 \mathrm{E}+00$ $\begin{array}{llll}3.6666667461395 \mathrm{E}+00 & 0.0000000000000 \mathrm{E}+00 & 0.0000000000000 \mathrm{E}+00 \quad 0.000 \mathrm{E}+00\end{array}$ $3.6666667461395 \mathrm{E}+00 \quad 0.0000000000000 \mathrm{E}+00 \quad 1.0000000000000 \mathrm{E}+00 \quad 0.000 \mathrm{E}+00$ $3.6666667461395 \mathrm{E}+00 \quad 1.0000000000000 \mathrm{E}+00 \quad 0.0000000000000 \mathrm{E}+00 \quad 0.000 \mathrm{E}+00$ $3.6666667461395 \mathrm{E}+001.0000000000000 \mathrm{E}+00 \quad 1.0000000000000 \mathrm{E}+00 \quad 0.000 \mathrm{E}+00$ $4.0000000000000 \mathrm{E}+00 \quad 0.0000000000000 \mathrm{E}+00 \quad 0.0000000000000 \mathrm{E}+00 \quad 0.000 \mathrm{E}+00$ $4.0000000000000 \mathrm{E}+00 \quad 0.0000000000000 \mathrm{E}+00 \quad 1.0000000000000 \mathrm{E}+00 \quad 0.000 \mathrm{E}+00$ $4.0000000000000 \mathrm{E}+00 \quad 1.0000000000000 \mathrm{E}+00 \quad 0.0000000000000 \mathrm{E}+00 \quad 0.000 \mathrm{E}+00$ $4.0000000000000 \mathrm{E}+001.0000000000000 \mathrm{E}+00 \quad 1.0000000000000 \mathrm{E}+00 \quad 0.000 \mathrm{E}+00$ $\begin{array}{llll}4.3333334922791 \mathrm{E}+00 & 0.0000000000000 \mathrm{E}+00 & 0.0000000000000 \mathrm{E}+00 & 0.000 \mathrm{E}+00\end{array}$ $\begin{array}{llll}4.3333334922791 \mathrm{E}+00 & 0.0000000000000 \mathrm{E}+00 & 1.0000000000000 \mathrm{E}+00 & 0.000 \mathrm{E}+00\end{array}$ $\begin{array}{llll}4.3333334922791 \mathrm{E}+00 & 1.0000000000000 \mathrm{E}+00 & 0.0000000000000 \mathrm{E}+00 & 0.000 \mathrm{E}+00\end{array}$ $4.3333334922791 \mathrm{E}+00 \quad 1.0000000000000 \mathrm{E}+00 \quad 1.0000000000000 \mathrm{E}+00 \quad 0.000 \mathrm{E}+00$ $\begin{array}{lllll}4.6666665077209 \mathrm{E}+00 & 0.0000000000000 \mathrm{E}+00 & 0.0000000000000 \mathrm{E}+00 & 0.000 \mathrm{E}+00\end{array}$ $\begin{array}{llll}4.6666665077209 \mathrm{E}+00 & 0.0000000000000 \mathrm{E}+00 & 1.0000000000000 \mathrm{E}+00 & 0.000 \mathrm{E}+00\end{array}$ $\begin{array}{llll}4.6666665077209 \mathrm{E}+00 & 1.0000000000000 \mathrm{E}+00 & 0.0000000000000 \mathrm{E}+00 & 0.000 \mathrm{E}+00\end{array}$ $4.6666665077209 \mathrm{E}+00 \quad 1.0000000000000 \mathrm{E}+00 \quad 1.0000000000000 \mathrm{E}+00 \quad 0.000 \mathrm{E}+00$ $5.0000000000000 \mathrm{E}+00 \quad 0.0000000000000 \mathrm{E}+00 \quad 0.0000000000000 \mathrm{E}+00 \quad 0.000 \mathrm{E}+00$ $5.0000000000000 \mathrm{E}+00 \quad 0.0000000000000 \mathrm{E}+00 \quad 1.0000000000000 \mathrm{E}+00 \quad 0.000 \mathrm{E}+00$ $5.0000000000000 \mathrm{E}+00 \quad 1.0000000000000 \mathrm{E}+00 \quad 0.0000000000000 \mathrm{E}+00 \quad 0.000 \mathrm{E}+00$ $5.0000000000000 \mathrm{E}+001.0000000000000 \mathrm{E}+00 \quad 1.0000000000000 \mathrm{E}+00 \quad 0.000 \mathrm{E}+00$ $\begin{array}{llll}5.3333334922791 \mathrm{E}+00 & 0.0000000000000 \mathrm{E}+00 & 0.0000000000000 \mathrm{E}+00 & 0.000 \mathrm{E}+00\end{array}$ $\begin{array}{llll}5.3333334922791 \mathrm{E}+00 & 0.0000000000000 \mathrm{E}+00 & 1.0000000000000 \mathrm{E}+00 & 0.000 \mathrm{E}+00\end{array}$ $5.3333334922791 \mathrm{E}+00 \quad 1.0000000000000 \mathrm{E}+00 \quad 0.0000000000000 \mathrm{E}+00 \quad 0.000 \mathrm{E}+00$ $5.3333334922791 \mathrm{E}+001.0000000000000 \mathrm{E}+00 \quad 1.0000000000000 \mathrm{E}+00 \quad 0.000 \mathrm{E}+00$ $\begin{array}{llll}5.6666669845581 \mathrm{E}+00 & 0.0000000000000 \mathrm{E}+00 & 0.0000000000000 \mathrm{E}+00 & 0.000 \mathrm{E}+00\end{array}$ $5.6666669845581 \mathrm{E}+00 \quad 0.0000000000000 \mathrm{E}+00 \quad 1.0000000000000 \mathrm{E}+00 \quad 0.000 \mathrm{E}+00$ $\begin{array}{llll}5.6666669845581 \mathrm{E}+00 & 1.0000000000000 \mathrm{E}+00 & 0.0000000000000 \mathrm{E}+00 & 0.000 \mathrm{E}+00\end{array}$ $5.6666669845581 \mathrm{E}+001.0000000000000 \mathrm{E}+00 \quad 1.0000000000000 \mathrm{E}+00 \quad 0.000 \mathrm{E}+00$ $6.0000009536743 \mathrm{E}+00 \quad 0.0000000000000 \mathrm{E}+00 \quad 0.0000000000000 \mathrm{E}+00 \quad 0.000 \mathrm{E}+00$ $6.0000009536743 \mathrm{E}+00 \quad 0.0000000000000 \mathrm{E}+00 \quad 1.0000000000000 \mathrm{E}+00 \quad 0.000 \mathrm{E}+00$ $6.0000009536743 \mathrm{E}+00 \quad 1.0000000000000 \mathrm{E}+00 \quad 0.0000000000000 \mathrm{E}+00 \quad 0.000 \mathrm{E}+00$ $6.0000009536743 \mathrm{E}+001.0000000000000 \mathrm{E}+00 \quad 1.0000000000000 \mathrm{E}+00 \quad 0.000 \mathrm{E}+00$ $6.3333344459534 \mathrm{E}+00 \quad 0.0000000000000 \mathrm{E}+00 \quad 0.0000000000000 \mathrm{E}+00 \quad 0.000 \mathrm{E}+00$ $6.3333344459534 \mathrm{E}+00 \quad 0.0000000000000 \mathrm{E}+00 \quad 1.0000000000000 \mathrm{E}+00 \quad 0.000 \mathrm{E}+00$ $6.3333344459534 \mathrm{E}+00 \quad 1.0000000000000 \mathrm{E}+00 \quad 0.0000000000000 \mathrm{E}+00 \quad 0.000 \mathrm{E}+00$ $6.3333344459534 \mathrm{E}+00 \quad 1.0000000000000 \mathrm{E}+00 \quad 1.0000000000000 \mathrm{E}+00 \quad 0.000 \mathrm{E}+00$ $\begin{array}{llll}6.6666679382324 \mathrm{E}+00 & 0.0000000000000 \mathrm{E}+00 & 0.0000000000000 \mathrm{E}+00 & 0.000 \mathrm{E}+00\end{array}$ $6.6666679382324 \mathrm{E}+00 \quad 0.0000000000000 \mathrm{E}+00 \quad 1.0000000000000 \mathrm{E}+00 \quad 0.000 \mathrm{E}+00$ 


\begin{tabular}{|c|c|c|c|c|c|c|c|c|c|c|}
\hline 83 & & \multicolumn{3}{|c|}{$6.6666679382324 \mathrm{E}+00$} & \multicolumn{4}{|c|}{$1.0000000000000 \mathrm{E}+00$} & $0.0000000000000 \mathrm{E}+00$ & $0.000 \mathrm{E}+00$ \\
\hline 84 & & \multicolumn{3}{|c|}{$6.6666679382324 \mathrm{E}+00$} & \multicolumn{4}{|c|}{$1.0000000000000 \mathrm{E}+00$} & $1.0000000000000 \mathrm{E}+00$ & $0.000 \mathrm{E}+00$ \\
\hline 85 & & \multicolumn{3}{|c|}{$7.0000019073486 \mathrm{E}+00$} & \multicolumn{4}{|c|}{$0.0000000000000 \mathrm{E}+00$} & $0.0000000000000 \mathrm{E}+00$ & $0.000 \mathrm{E}+00$ \\
\hline 86 & & \multicolumn{3}{|c|}{$7.0000019073486 \mathrm{E}+00$} & \multicolumn{4}{|c|}{$0.0000000000000 \mathrm{E}+00$} & $1.0000000000000 \mathrm{E}+00$ & $0.000 \mathrm{E}+00$ \\
\hline 87 & & \multicolumn{3}{|c|}{$7.0000019073486 \mathrm{E}+00$} & \multicolumn{4}{|c|}{$1.0000000000000 \mathrm{E}+00$} & $0.0000000000000 \mathrm{E}+00$ & $0.000 \mathrm{E}+00$ \\
\hline 88 & & \multicolumn{3}{|c|}{$7.0000019073486 \mathrm{E}+00$} & \multicolumn{4}{|c|}{$1.0000000000000 \mathrm{E}+00$} & $1.0000000000000 \mathrm{E}+00$ & $0.000 \mathrm{E}+00$ \\
\hline 89 & & 7.33333539 & 5277 & +00 & 0.0000 & 000 & 00001 & +00 & $0.0000000000000 \mathrm{E}+00$ & $0.000 \mathrm{E}+00$ \\
\hline 90 & & 7.333335399 & 5277 & +00 & 0.0000 & 000 & 00001 & +00 & $1.0000000000000 \mathrm{E}+00$ & $0.000 \mathrm{E}+00$ \\
\hline 91 & & 7.33333539 & 5277 & +00 & 1.0000 & 000 & 00001 & +00 & $0.0000000000000 \mathrm{E}+00$ & $0.000 \mathrm{E}+00$ \\
\hline 92 & & 7.33333539 & 5277 & +00 & 1.0000 & 000 & 00001 & +00 & $1.0000000000000 \mathrm{E}+00$ & $0.000 \mathrm{E}+00$ \\
\hline 93 & & 7.666668891 & 9067 & +00 & 0.0000 & 000 & 00001 & +00 & $0.0000000000000 \mathrm{E}+00$ & $0.000 \mathrm{E}+00$ \\
\hline 94 & & 7.666668891 & 9067 & +00 & 0.0000 & 000 & 00001 & +00 & $1.0000000000000 \mathrm{E}+00$ & $0.000 \mathrm{E}+00$ \\
\hline 95 & & 7.666668891 & 9067 & +00 & 1.0000 & 000 & 00001 & +00 & $0.0000000000000 \mathrm{E}+00$ & $0.000 \mathrm{E}+00$ \\
\hline 96 & & 7.666668891 & 9067 & +00 & 1.0000 & 000 & 00001 & +00 & $1.0000000000000 \mathrm{E}+00$ & $0.000 \mathrm{E}+00$ \\
\hline 97 & & 8.000002861 & 229 & +00 & 0.0000 & 000 & 00001 & +00 & $0.0000000000000 \mathrm{E}+00$ & $0.000 \mathrm{E}+00$ \\
\hline 98 & & 8.000002861 & 229 & +00 & 0.0000 & 000 & 00001 & +00 & $1.0000000000000 \mathrm{E}+00$ & $0.000 \mathrm{E}+00$ \\
\hline 99 & & 8.000002861 & 229 & +00 & 1.0000 & 000 & 00001 & +00 & $0.0000000000000 \mathrm{E}+00$ & $0.000 \mathrm{E}+00$ \\
\hline 100 & & 8.000002861 & 229 & +00 & 1.0000 & 000 & 00001 & +00 & $1.0000000000000 \mathrm{E}+00$ & $0.000 \mathrm{E}+00$ \\
\hline 101 & & 8.333335876 & 1648 & +00 & 0.0000 & 000 & 00001 & +00 & $0.0000000000000 \mathrm{E}+00$ & $0.000 \mathrm{E}+00$ \\
\hline 102 & & 8.333335876 & 1648 & +00 & 0.0000 & 000 & 00001 & +00 & $1.0000000000000 \mathrm{E}+00$ & $0.000 \mathrm{E}+00$ \\
\hline 103 & & 8.333335876 & 1648 & +00 & 1.0000 & 000 & 00001 & +00 & $0.0000000000000 \mathrm{E}+00$ & $0.000 \mathrm{E}+00$ \\
\hline 104 & & 8.333335876 & 1648 & +00 & 1.0000 & 000 & 00001 & +00 & $1.0000000000000 \mathrm{E}+00$ & $0.000 \mathrm{E}+00$ \\
\hline 105 & & 8.666669845 & 5811 & +00 & 0.0000 & 000 & 00001 & +00 & $0.0000000000000 \mathrm{E}+00$ & $0.000 \mathrm{E}+00$ \\
\hline 106 & & 8.666669845 & 5811 & +00 & 0.0000 & 000 & 00001 & +00 & $1.0000000000000 \mathrm{E}+00$ & $0.000 \mathrm{E}+00$ \\
\hline 107 & & 8.666669845 & 5811 & +00 & 1.0000 & 000 & 00001 & +00 & $0.0000000000000 \mathrm{E}+00$ & $0.000 \mathrm{E}+00$ \\
\hline 108 & & 8.666669845 & 5811 & +00 & 1.0000 & 000 & 00001 & +00 & $1.0000000000000 \mathrm{E}+00$ & $0.000 \mathrm{E}+00$ \\
\hline 109 & & 9.000003814 & 5973 & +00 & 0.0000 & 000 & 00001 & +00 & $0.0000000000000 \mathrm{E}+00$ & $0.000 \mathrm{E}+00$ \\
\hline 110 & & 9.000003814 & 5973 & +00 & 0.0000 & 000 & 00001 & +00 & $1.0000000000000 \mathrm{E}+00$ & $0.000 \mathrm{E}+00$ \\
\hline 111 & & 9.00000381 & 5973 & +00 & 1.0000 & 000 & 00001 & +00 & $0.0000000000000 \mathrm{E}+00$ & $0.000 \mathrm{E}+00$ \\
\hline 112 & & 9.000003814 & 5973 & +00 & 1.0000 & 000 & 00001 & +00 & $1.0000000000000 \mathrm{E}+00$ & $0.000 \mathrm{E}+00$ \\
\hline 113 & & 9.333336830 & 392 & +00 & 0.0000 & 000 & 00001 & +00 & $0.0000000000000 \mathrm{E}+00$ & $0.000 \mathrm{E}+00$ \\
\hline 114 & & 9.333336830 & 392 & +00 & 0.0000 & 000 & 00001 & +00 & $1.0000000000000 \mathrm{E}+00$ & $0.000 \mathrm{E}+00$ \\
\hline 115 & & 9.333336830 & 392 & +00 & 1.0000 & 000 & 00001 & +00 & $0.0000000000000 \mathrm{E}+00$ & $0.000 \mathrm{E}+00$ \\
\hline 116 & & 9.333336830 & 392 & +00 & 1.0000 & 000 & 00001 & +00 & $1.0000000000000 \mathrm{E}+00$ & $0.000 \mathrm{E}+00$ \\
\hline 117 & & 9.66667079 & 2554 & +00 & 0.0000 & 000 & 00001 & +00 & $0.0000000000000 \mathrm{E}+00$ & $0.000 \mathrm{E}+00$ \\
\hline 118 & & 9.66667079 & 2554 & +00 & 0.0000 & 000 & 00001 & +00 & $1.0000000000000 \mathrm{E}+00$ & $0.000 \mathrm{E}+00$ \\
\hline 119 & & 9.666670799 & 2554 & +00 & 1.0000 & 000 & 00001 & +00 & $0.0000000000000 \mathrm{E}+00$ & $0.000 \mathrm{E}+00$ \\
\hline 120 & & 9.66667079 & 2554 & +00 & 1.0000 & 000 & 00001 & +00 & $1.0000000000000 \mathrm{E}+00$ & $0.000 \mathrm{E}+00$ \\
\hline 121 & & 1.000000000 & 000 & +01 & 0.0000 & 000 & 00001 & +00 & $0.0000000000000 \mathrm{E}+00$ & $0.000 \mathrm{E}+00$ \\
\hline 122 & & 1.000000000 & 000 & +01 & 0.0000 & 000 & 00001 & +00 & $1.0000000000000 \mathrm{E}+00$ & $0.000 \mathrm{E}+00$ \\
\hline 123 & & 1.000000000 & 000 & +01 & 1.0000 & 000 & 00001 & +00 & $0.0000000000000 \mathrm{E}+00$ & $0.000 \mathrm{E}+00$ \\
\hline 124 & & 1.000000000 & 000 & +01 & 1.0000 & 000 & 00001 & +00 & $1.0000000000000 \mathrm{E}+00$ & $0.000 \mathrm{E}+00$ \\
\hline 1 & 1 & 1 & 5 & 7 & 3 & 2 & 6 & 8 & 4 & \\
\hline 2 & 1 & 5 & 9 & 11 & 7 & 6 & 10 & 12 & 8 & \\
\hline 3 & 1 & 9 & 13 & 15 & 11 & 10 & 14 & 16 & 12 & \\
\hline 4 & 1 & 13 & 17 & 19 & 15 & 14 & 18 & 20 & 16 & \\
\hline 5 & 1 & 17 & 21 & 23 & 19 & 18 & 22 & 24 & 20 & \\
\hline 6 & 1 & 21 & 25 & 27 & 23 & 22 & 26 & 28 & 24 & \\
\hline 7 & 1 & 25 & 29 & 31 & 27 & 26 & 30 & 32 & 28 & \\
\hline 8 & 1 & 29 & 33 & 35 & 31 & 30 & 34 & 36 & 32 & \\
\hline 9 & 1 & 33 & 37 & 39 & 35 & 34 & 38 & 40 & 36 & \\
\hline 10 & 1 & 37 & 41 & 43 & 39 & 38 & 42 & 44 & 40 & \\
\hline 11 & 1 & 41 & 45 & 47 & 43 & 42 & 46 & 48 & 44 & \\
\hline 12 & 1 & 45 & 49 & 51 & 47 & 46 & 50 & 52 & 48 & \\
\hline 13 & 1 & 49 & 53 & 55 & 51 & 50 & 54 & 56 & 52 & \\
\hline 14 & 1 & 53 & 57 & 59 & 55 & 54 & 58 & 60 & 56 & \\
\hline 15 & 1 & 57 & 61 & 63 & 59 & 58 & 62 & 64 & 60 & \\
\hline 16 & 1 & 61 & 65 & 67 & 63 & 62 & 66 & 68 & 64 & \\
\hline 17 & 1 & 65 & 69 & 71 & 67 & 66 & 70 & 72 & 68 & \\
\hline 18 & 1 & 69 & 73 & 75 & 71 & 70 & 74 & 76 & 72 & \\
\hline 19 & 1 & 73 & 77 & 79 & 75 & 74 & 78 & 80 & 76 & \\
\hline 20 & 1 & 77 & 81 & 83 & 79 & 78 & 82 & 84 & 80 & \\
\hline 21 & 1 & 81 & 85 & 87 & 83 & 82 & 86 & 88 & 84 & \\
\hline 22 & 1 & 85 & 89 & 91 & 87 & 86 & 90 & 92 & 88 & \\
\hline
\end{tabular}




\begin{tabular}{|c|c|c|c|c|c|c|c|c|c|c|c|}
\hline 23 & 1 & & 89 & 93 & 95 & 91 & 90 & 94 & 96 & 92 & \\
\hline 24 & 1 & & 93 & 97 & 99 & 95 & 94 & 98 & 100 & 96 & \\
\hline 25 & 1 & & 97 & 101 & 103 & 99 & 98 & 102 & 104 & 100 & \\
\hline 26 & 1 & & 101 & 105 & 107 & 103 & 102 & 106 & 108 & 104 & \\
\hline 27 & 1 & & 105 & 109 & 111 & 107 & 106 & 110 & 112 & 108 & \\
\hline 28 & 1 & & 109 & 113 & 115 & 111 & 110 & 114 & 116 & 112 & \\
\hline 29 & 1 & & 113 & 117 & 119 & 115 & 114 & 118 & 120 & 116 & \\
\hline 30 & 1 & & 117 & 121 & 123 & 119 & 118 & 122 & 124 & 120 & \\
\hline 1 & 1 & 2 & 4 & 3 & 0 & $1.000 \mathrm{E}$ & +00 & 0 & 3.000 & $\mathrm{E}+01$ & $0.000 \mathrm{E}+00$ \\
\hline
\end{tabular}

\subsection{Constant Slide Line}

Problem: $\quad$ This models the transient linear flow of heat in a semi-infinite solid with a temperature boundary condition specified at $\mathrm{x}=0$ and a specified global initial temperature condition. Note that the left side of the interface is defined as surface A and the right as surface B.

Analytic solution: Because an analytical solution to this problem could not easily obtained, the solution to this problem used as a reference was that of a transient conduction problem in a semi-infinite solid. The mesh was created so as to create a small block at $x=2.95$ and $x=3.05$ for a solid of length $\mathrm{L}=10$. Then, the same problem could be repeated removing that block and inserting a slide line in its place, with $\mathrm{x}=2.95$ representing surface $\mathrm{A}$ and $\mathrm{x}=3.05$ surface $\mathrm{B}$. The conductance of the slide line was given as $\mathrm{H}=\mathrm{k} / \mathrm{th}$ (where th is the thickness of the small block), and then the solutions at surfaces A and B were compared.

Case parameters:

$$
\begin{array}{lrl}
\text { Slab length - } & L & =10 \\
\text { Gap thickness - } & \text { th } & =0.1 \\
\text { Boundary conditions } & T_{o}=0 \text { at } x=0 \\
& T_{i}=0 \\
& H=10 \\
\text { Time - } & t=3
\end{array}
$$

Results: $\quad \underline{\text { Surface }} \quad \underline{\mathrm{T} \text { (ref.) }} \quad \underline{\mathrm{T} \text { (slide line) }}$

$\begin{array}{lll}\text { A } & 7.71602 & 7.63951 \\ \text { B } & 7.86990 & 7.79339\end{array}$

Input file:

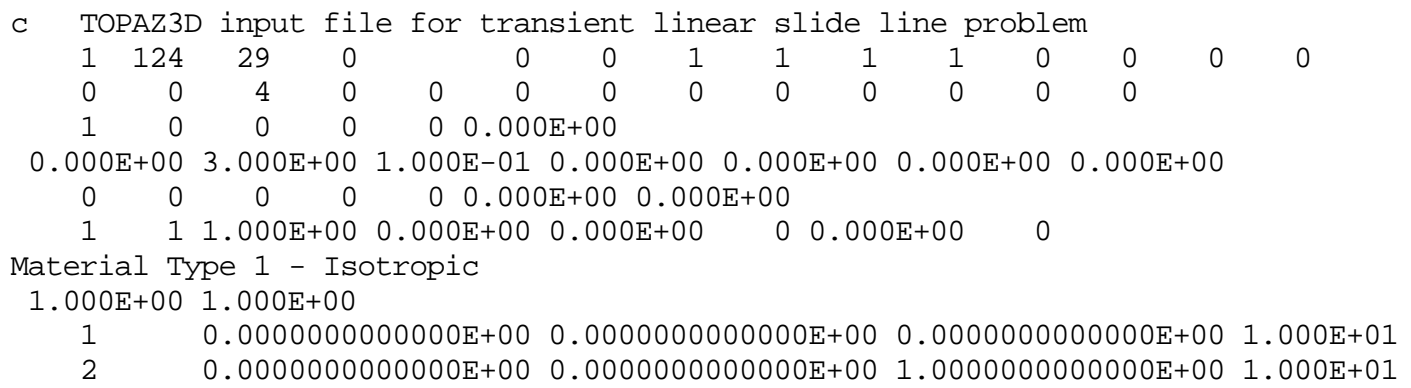


$0.0000000000000 \mathrm{E}+00 \quad 1.0000000000000 \mathrm{E}+00 \quad 0.0000000000000 \mathrm{E}+00 \quad 1.000 \mathrm{E}+01$ $0.0000000000000 \mathrm{E}+001.0000000000000 \mathrm{E}+00 \quad 1.0000000000000 \mathrm{E}+00 \quad 1.000 \mathrm{E}+01$ $2.9500001668930 \mathrm{E}-01 \quad 0.0000000000000 \mathrm{E}+00 \quad 0.0000000000000 \mathrm{E}+00 \quad 1.000 \mathrm{E}+01$ $2.9500001668930 \mathrm{E}-01 \quad 0.0000000000000 \mathrm{E}+00 \quad 1.0000000000000 \mathrm{E}+00 \quad 1.000 \mathrm{E}+01$ $2.9500001668930 \mathrm{E}-01 \quad 1.0000000000000 \mathrm{E}+00 \quad 0.0000000000000 \mathrm{E}+00 \quad 1.000 \mathrm{E}+01$ $2.9500001668930 \mathrm{E}-011.0000000000000 \mathrm{E}+00 \quad 1.0000000000000 \mathrm{E}+00 \quad 1.000 \mathrm{E}+01$ $5.9000003337860 \mathrm{E}-01 \quad 0.0000000000000 \mathrm{E}+00 \quad 0.0000000000000 \mathrm{E}+00 \quad 1.000 \mathrm{E}+01$ $\begin{array}{llll}5.9000003337860 \mathrm{E}-01 & 0.0000000000000 \mathrm{E}+00 & 1.0000000000000 \mathrm{E}+00 & 1.000 \mathrm{E}+01\end{array}$ $5.9000003337860 \mathrm{E}-01 \quad 1.0000000000000 \mathrm{E}+00 \quad 0.0000000000000 \mathrm{E}+00 \quad 1.000 \mathrm{E}+01$ $5.9000003337860 \mathrm{E}-01 \quad 1.0000000000000 \mathrm{E}+00 \quad 1.0000000000000 \mathrm{E}+00 \quad 1.000 \mathrm{E}+01$ $8.8500005006790 \mathrm{E}-01 \quad 0.0000000000000 \mathrm{E}+00 \quad 0.0000000000000 \mathrm{E}+00 \quad 1.000 \mathrm{E}+01$ $8.8500005006790 \mathrm{E}-01 \quad 0.0000000000000 \mathrm{E}+00 \quad 1.0000000000000 \mathrm{E}+00 \quad 1.000 \mathrm{E}+01$ $8.8500005006790 \mathrm{E}-01 \quad 1.0000000000000 \mathrm{E}+00 \quad 0.0000000000000 \mathrm{E}+00 \quad 1.000 \mathrm{E}+01$ $8.8500005006790 \mathrm{E}-01 \quad 1.0000000000000 \mathrm{E}+00 \quad 1.0000000000000 \mathrm{E}+00 \quad 1.000 \mathrm{E}+01$ $1.1800000667572 \mathrm{E}+00 \quad 0.0000000000000 \mathrm{E}+00 \quad 0.0000000000000 \mathrm{E}+00 \quad 1.000 \mathrm{E}+01$ $1.1800000667572 \mathrm{E}+00 \quad 0.0000000000000 \mathrm{E}+00 \quad 1.0000000000000 \mathrm{E}+00 \quad 1.000 \mathrm{E}+01$ $1.1800000667572 \mathrm{E}+00 \quad 1.0000000000000 \mathrm{E}+00 \quad 0.0000000000000 \mathrm{E}+00 \quad 1.000 \mathrm{E}+01$ $1.1800000667572 \mathrm{E}+00 \quad 1.0000000000000 \mathrm{E}+00 \quad 1.0000000000000 \mathrm{E}+00 \quad 1.000 \mathrm{E}+01$ $1.4750000238419 \mathrm{E}+00 \quad 0.0000000000000 \mathrm{E}+00 \quad 0.0000000000000 \mathrm{E}+00 \quad 1.000 \mathrm{E}+01$ $1.4750000238419 \mathrm{E}+00 \quad 0.0000000000000 \mathrm{E}+00 \quad 1.0000000000000 \mathrm{E}+00 \quad 1.000 \mathrm{E}+01$ $1.4750000238419 \mathrm{E}+00 \quad 1.0000000000000 \mathrm{E}+00 \quad 0.0000000000000 \mathrm{E}+00 \quad 1.000 \mathrm{E}+01$ $1.4750000238419 \mathrm{E}+00 \quad 1.0000000000000 \mathrm{E}+00 \quad 1.0000000000000 \mathrm{E}+00 \quad 1.000 \mathrm{E}+01$ $1.7700001001358 \mathrm{E}+00 \quad 0.0000000000000 \mathrm{E}+00 \quad 0.0000000000000 \mathrm{E}+00 \quad 1.000 \mathrm{E}+01$ $1.7700001001358 \mathrm{E}+00 \quad 0.0000000000000 \mathrm{E}+00 \quad 1.0000000000000 \mathrm{E}+00 \quad 1.000 \mathrm{E}+01$ $1.7700001001358 \mathrm{E}+00 \quad 1.0000000000000 \mathrm{E}+00 \quad 0.0000000000000 \mathrm{E}+00 \quad 1.000 \mathrm{E}+01$ $1.7700001001358 \mathrm{E}+00 \quad 1.0000000000000 \mathrm{E}+00 \quad 1.0000000000000 \mathrm{E}+00 \quad 1.000 \mathrm{E}+01$ $2.0650000572205 \mathrm{E}+00 \quad 0.0000000000000 \mathrm{E}+00 \quad 0.0000000000000 \mathrm{E}+00 \quad 1.000 \mathrm{E}+01$ $2.0650000572205 \mathrm{E}+00 \quad 0.0000000000000 \mathrm{E}+00 \quad 1.0000000000000 \mathrm{E}+00 \quad 1.000 \mathrm{E}+01$ $2.0650000572205 \mathrm{E}+001.0000000000000 \mathrm{E}+00 \quad 0.0000000000000 \mathrm{E}+00 \quad 1.000 \mathrm{E}+01$ $2.0650000572205 \mathrm{E}+00 \quad 1.0000000000000 \mathrm{E}+00 \quad 1.0000000000000 \mathrm{E}+00 \quad 1.000 \mathrm{E}+01$ $2.3600001335144 \mathrm{E}+00 \quad 0.0000000000000 \mathrm{E}+00 \quad 0.0000000000000 \mathrm{E}+00 \quad 1.000 \mathrm{E}+01$ $2.3600001335144 \mathrm{E}+00 \quad 0.0000000000000 \mathrm{E}+00 \quad 1.0000000000000 \mathrm{E}+00 \quad 1.000 \mathrm{E}+01$ $2.3600001335144 \mathrm{E}+00 \quad 1.0000000000000 \mathrm{E}+00 \quad 0.0000000000000 \mathrm{E}+00 \quad 1.000 \mathrm{E}+01$ $2.3600001335144 \mathrm{E}+001.0000000000000 \mathrm{E}+00 \quad 1.0000000000000 \mathrm{E}+00 \quad 1.000 \mathrm{E}+01$ $2.6550002098083 \mathrm{E}+00 \quad 0.0000000000000 \mathrm{E}+00 \quad 0.0000000000000 \mathrm{E}+00 \quad 1.000 \mathrm{E}+01$ $2.6550002098083 \mathrm{E}+00 \quad 0.0000000000000 \mathrm{E}+00 \quad 1.0000000000000 \mathrm{E}+00 \quad 1.000 \mathrm{E}+01$ $2.6550002098083 \mathrm{E}+00 \quad 1.0000000000000 \mathrm{E}+00 \quad 0.0000000000000 \mathrm{E}+00 \quad 1.000 \mathrm{E}+01$ $2.6550002098083 \mathrm{E}+00 \quad 1.0000000000000 \mathrm{E}+00 \quad 1.0000000000000 \mathrm{E}+00 \quad 1.000 \mathrm{E}+01$ $2.9500000476837 \mathrm{E}+00 \quad 0.0000000000000 \mathrm{E}+00 \quad 0.0000000000000 \mathrm{E}+00 \quad 1.000 \mathrm{E}+01$ $2.9500000476837 \mathrm{E}+00 \quad 0.0000000000000 \mathrm{E}+00 \quad 1.0000000000000 \mathrm{E}+00 \quad 1.000 \mathrm{E}+01$ $2.9500000476837 \mathrm{E}+00 \quad 1.0000000000000 \mathrm{E}+00 \quad 0.0000000000000 \mathrm{E}+00 \quad 1.000 \mathrm{E}+01$ $2.9500000476837 \mathrm{E}+00 \quad 1.0000000000000 \mathrm{E}+00 \quad 1.0000000000000 \mathrm{E}+00 \quad 1.000 \mathrm{E}+01$ $3.0499999523163 \mathrm{E}+00 \quad 0.0000000000000 \mathrm{E}+00 \quad 0.0000000000000 \mathrm{E}+00 \quad 1.000 \mathrm{E}+01$ $3.0499999523163 \mathrm{E}+00 \quad 0.0000000000000 \mathrm{E}+00 \quad 1.0000000000000 \mathrm{E}+00 \quad 1.000 \mathrm{E}+01$ $3.0499999523163 \mathrm{E}+00 \quad 1.0000000000000 \mathrm{E}+00 \quad 0.0000000000000 \mathrm{E}+00 \quad 1.000 \mathrm{E}+01$ $3.0499999523163 \mathrm{E}+00 \quad 1.0000000000000 \mathrm{E}+00 \quad 1.0000000000000 \mathrm{E}+00 \quad 1.000 \mathrm{E}+01$ $3.4157893657684 \mathrm{E}+00 \quad 0.0000000000000 \mathrm{E}+00 \quad 0.0000000000000 \mathrm{E}+00 \quad 1.000 \mathrm{E}+01$ $3.4157893657684 \mathrm{E}+00 \quad 0.0000000000000 \mathrm{E}+00 \quad 1.0000000000000 \mathrm{E}+00 \quad 1.000 \mathrm{E}+01$ $3.4157893657684 \mathrm{E}+00 \quad 1.0000000000000 \mathrm{E}+00 \quad 0.0000000000000 \mathrm{E}+00 \quad 1.000 \mathrm{E}+01$ $3.4157893657684 \mathrm{E}+00 \quad 1.0000000000000 \mathrm{E}+00 \quad 1.0000000000000 \mathrm{E}+00 \quad 1.000 \mathrm{E}+01$ $3.7815790176392 \mathrm{E}+00 \quad 0.0000000000000 \mathrm{E}+00 \quad 0.0000000000000 \mathrm{E}+00 \quad 1.000 \mathrm{E}+01$ $3.7815790176392 \mathrm{E}+00 \quad 0.0000000000000 \mathrm{E}+00 \quad 1.0000000000000 \mathrm{E}+00 \quad 1.000 \mathrm{E}+01$ $3.7815790176392 \mathrm{E}+00 \quad 1.0000000000000 \mathrm{E}+00 \quad 0.0000000000000 \mathrm{E}+00 \quad 1.000 \mathrm{E}+01$ $3.7815790176392 \mathrm{E}+001.0000000000000 \mathrm{E}+00 \quad 1.0000000000000 \mathrm{E}+00 \quad 1.000 \mathrm{E}+01$ $4.1473684310913 \mathrm{E}+00 \quad 0.0000000000000 \mathrm{E}+00 \quad 0.0000000000000 \mathrm{E}+00 \quad 1.000 \mathrm{E}+01$ $4.1473684310913 \mathrm{E}+00 \quad 0.0000000000000 \mathrm{E}+00 \quad 1.0000000000000 \mathrm{E}+00 \quad 1.000 \mathrm{E}+01$ $4.1473684310913 \mathrm{E}+00 \quad 1.0000000000000 \mathrm{E}+00 \quad 0.0000000000000 \mathrm{E}+00 \quad 1.000 \mathrm{E}+01$ $4.1473684310913 \mathrm{E}+00 \quad 1.0000000000000 \mathrm{E}+00 \quad 1.0000000000000 \mathrm{E}+00 \quad 1.000 \mathrm{E}+01$ $4.5131578445435 \mathrm{E}+00 \quad 0.0000000000000 \mathrm{E}+00 \quad 0.0000000000000 \mathrm{E}+00 \quad 1.000 \mathrm{E}+01$ $4.5131578445435 \mathrm{E}+00 \quad 0.0000000000000 \mathrm{E}+00 \quad 1.0000000000000 \mathrm{E}+00 \quad 1.000 \mathrm{E}+01$ $4.5131578445435 \mathrm{E}+00 \quad 1.0000000000000 \mathrm{E}+00 \quad 0.0000000000000 \mathrm{E}+00 \quad 1.000 \mathrm{E}+01$ $4.5131578445435 \mathrm{E}+00 \quad 1.0000000000000 \mathrm{E}+00 \quad 1.0000000000000 \mathrm{E}+00 \quad 1.000 \mathrm{E}+01$ $\begin{array}{lllllll}4.8789472579956 \mathrm{E}+00 & 0.0000000000000 \mathrm{E}+00 & 0.0000000000000 \mathrm{E}+00 & 1.000 \mathrm{E}+01\end{array}$ $4.8789472579956 \mathrm{E}+00 \quad 0.0000000000000 \mathrm{E}+00 \quad 1.0000000000000 \mathrm{E}+00 \quad 1.000 \mathrm{E}+01$ 


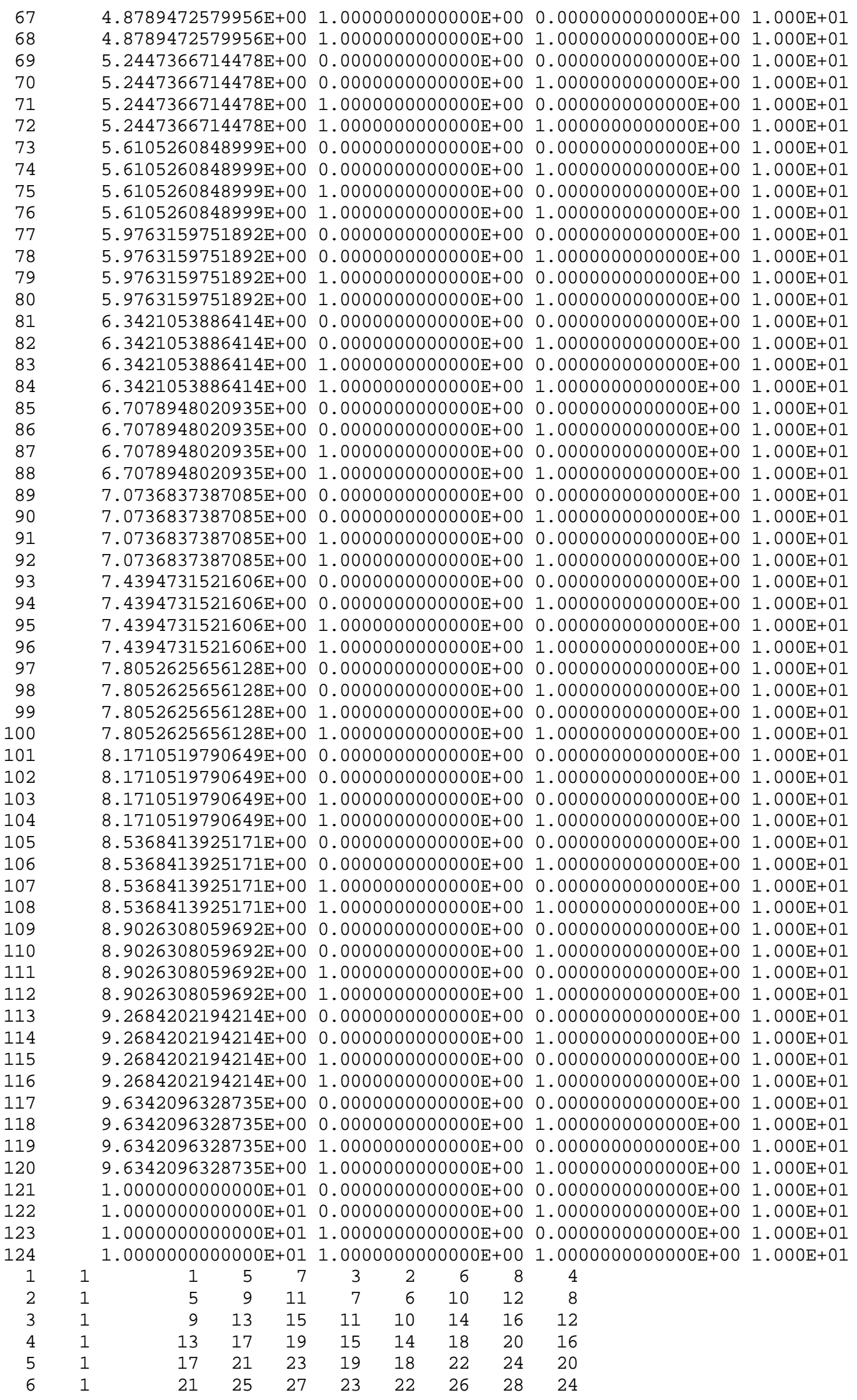




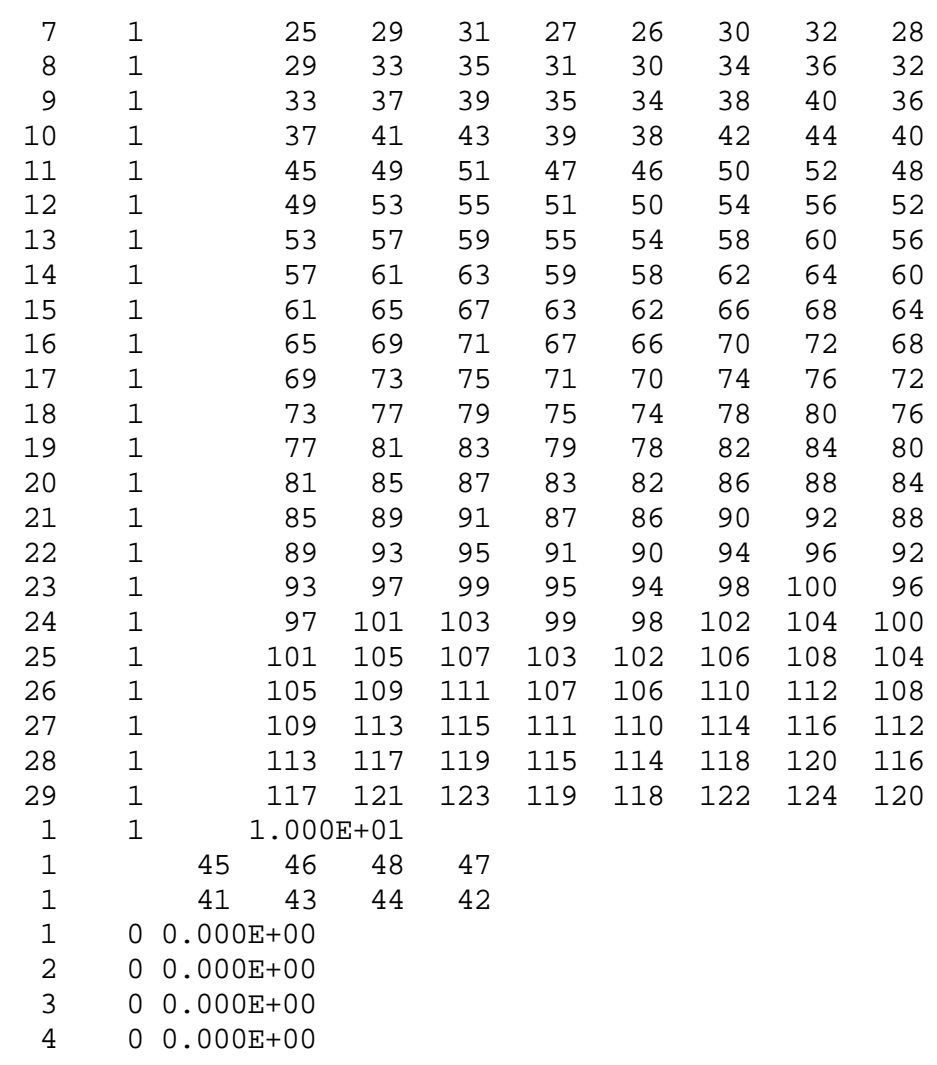

\section{Constant Special Internal Element}

Problem: $\quad$ This models the transient linear flow of heat in a semi-infinite solid with a gap. A temperature boundary condition at $\mathrm{x}=0$ and a global initial temperature condition are specified. Note that the left side of the gap is defined as surface A and the right as surface B.

Analytic solution: $\quad$ Because an analytical solution to this problem could not easily obtained, the solution to this problem used as a reference was that of a transient conduction problem in a semi-infinite solid. The mesh was created so as to create a small block at $x=2.95$ and $x=3.05$ for a solid of length $\mathrm{L}=10$. Then, the same problem could be repeated removing that block and inserting a special internal element in its place, with $\mathrm{x}=2.95$ representing surface $\mathrm{A}$ and $\mathrm{x}=3.05$ surface $\mathrm{B}$.

The conductance across the gap was given as $\mathrm{f}=\mathrm{k} / \mathrm{th}$ (where th is the thickness of the small block), and then the solutions at surfaces A and $\mathrm{B}$ were compared.

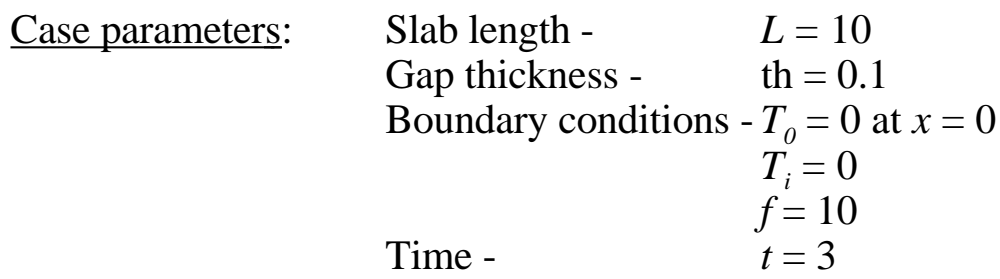


Results:

$\underline{\text { Surface }} \quad \underline{\mathrm{T} \text { (ref.) }} \quad \underline{\mathrm{T} \text { (internal element) }}$

$\begin{array}{lll}\text { A } & 7.71602 & 7.63951 \\ \text { B } & 7.86990 & 7.79339\end{array}$

\section{Input file:}

C TOPAZ3D input file for transient linear internal element problem

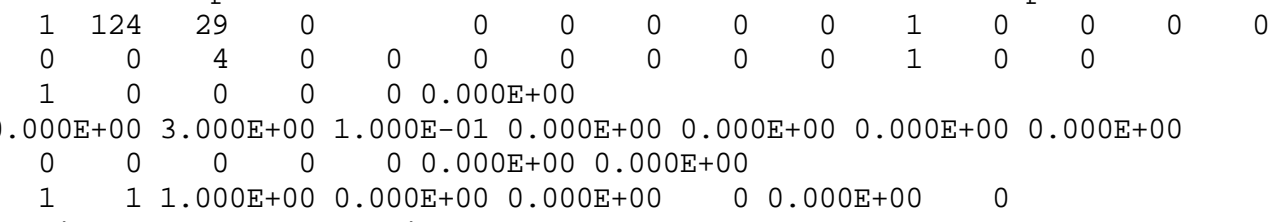

Material Type 1 - Isotropic

$1.000 \mathrm{E}+001.000 \mathrm{E}+00$

$1 \quad 0.0000000000000 \mathrm{E}+00 \quad 0.0000000000000 \mathrm{E}+00 \quad 0.0000000000000 \mathrm{E}+00 \quad 1.000 \mathrm{E}+01$

$2 \quad 0.0000000000000 \mathrm{E}+00 \quad 0.0000000000000 \mathrm{E}+00 \quad 1.0000000000000 \mathrm{E}+001.000 \mathrm{E}+01$

$3 \quad 0.0000000000000 \mathrm{E}+00 \quad 1.0000000000000 \mathrm{E}+00 \quad 0.0000000000000 \mathrm{E}+001.000 \mathrm{E}+01$

$4 \quad 0.0000000000000 \mathrm{E}+001.0000000000000 \mathrm{E}+001.0000000000000 \mathrm{E}+001.000 \mathrm{E}+01$

$5 \quad 2.9500001668930 \mathrm{E}-01 \quad 0.0000000000000 \mathrm{E}+00 \quad 0.0000000000000 \mathrm{E}+00 \quad 1.000 \mathrm{E}+01$

$6 \quad 2.9500001668930 \mathrm{E}-01 \quad 0.0000000000000 \mathrm{E}+00 \quad 1.0000000000000 \mathrm{E}+00 \quad 1.000 \mathrm{E}+01$

$7 \quad 2.9500001668930 \mathrm{E}-01 \quad 1.0000000000000 \mathrm{E}+00 \quad 0.0000000000000 \mathrm{E}+001.000 \mathrm{E}+01$

$8 \quad 2.9500001668930 \mathrm{E}-01 \quad 1.0000000000000 \mathrm{E}+00 \quad 1.0000000000000 \mathrm{E}+001.000 \mathrm{E}+01$

$95.9000003337860 \mathrm{E}-01 \quad 0.0000000000000 \mathrm{E}+00 \quad 0.0000000000000 \mathrm{E}+00 \quad 1.000 \mathrm{E}+01$

$105.9000003337860 \mathrm{E}-01 \quad 0.0000000000000 \mathrm{E}+00 \quad 1.0000000000000 \mathrm{E}+00 \quad 1.000 \mathrm{E}+01$

$115.9000003337860 \mathrm{E}-01 \quad 1.0000000000000 \mathrm{E}+00 \quad 0.0000000000000 \mathrm{E}+001.000 \mathrm{E}+01$

$125.9000003337860 \mathrm{E}-01 \quad 1.0000000000000 \mathrm{E}+00 \quad 1.0000000000000 \mathrm{E}+00 \quad 1.000 \mathrm{E}+01$

$138.8500005006790 \mathrm{E}-01 \quad 0.0000000000000 \mathrm{E}+00 \quad 0.0000000000000 \mathrm{E}+00 \quad 1.000 \mathrm{E}+01$

$148.8500005006790 \mathrm{E}-01 \quad 0.0000000000000 \mathrm{E}+00 \quad 1.0000000000000 \mathrm{E}+001.000 \mathrm{E}+01$

$158.8500005006790 \mathrm{E}-01 \quad 1.0000000000000 \mathrm{E}+00 \quad 0.0000000000000 \mathrm{E}+001.000 \mathrm{E}+01$

$168.8500005006790 \mathrm{E}-01 \quad 1.0000000000000 \mathrm{E}+00 \quad 1.0000000000000 \mathrm{E}+001.000 \mathrm{E}+01$

$171.1800000667572 \mathrm{E}+00 \quad 0.0000000000000 \mathrm{E}+00 \quad 0.0000000000000 \mathrm{E}+00 \quad 1.000 \mathrm{E}+01$

$181.1800000667572 \mathrm{E}+00 \quad 0.0000000000000 \mathrm{E}+00 \quad 1.0000000000000 \mathrm{E}+00 \quad 1.000 \mathrm{E}+01$

$191.1800000667572 \mathrm{E}+00 \quad 1.0000000000000 \mathrm{E}+00 \quad 0.0000000000000 \mathrm{E}+00 \quad 1.000 \mathrm{E}+01$

$201.1800000667572 \mathrm{E}+00 \quad 1.0000000000000 \mathrm{E}+00 \quad 1.0000000000000 \mathrm{E}+00 \quad 1.000 \mathrm{E}+01$

$21 \quad 1.4750000238419 \mathrm{E}+00 \quad 0.0000000000000 \mathrm{E}+00 \quad 0.0000000000000 \mathrm{E}+00 \quad 1.000 \mathrm{E}+01$

$221.4750000238419 \mathrm{E}+00 \quad 0.0000000000000 \mathrm{E}+00 \quad 1.0000000000000 \mathrm{E}+00 \quad 1.000 \mathrm{E}+01$

$231.4750000238419 \mathrm{E}+00 \quad 1.0000000000000 \mathrm{E}+00 \quad 0.0000000000000 \mathrm{E}+00 \quad 1.000 \mathrm{E}+01$

$241.4750000238419 \mathrm{E}+001.0000000000000 \mathrm{E}+00 \quad 1.0000000000000 \mathrm{E}+001.000 \mathrm{E}+01$

$25 \quad 1.7700001001358 \mathrm{E}+00 \quad 0.0000000000000 \mathrm{E}+00 \quad 0.0000000000000 \mathrm{E}+00 \quad 1.000 \mathrm{E}+01$

$261.7700001001358 \mathrm{E}+00 \quad 0.0000000000000 \mathrm{E}+00 \quad 1.0000000000000 \mathrm{E}+001.000 \mathrm{E}+01$

$271.7700001001358 \mathrm{E}+001.0000000000000 \mathrm{E}+00 \quad 0.0000000000000 \mathrm{E}+001.000 \mathrm{E}+01$

$281.7700001001358 \mathrm{E}+001.0000000000000 \mathrm{E}+00 \quad 1.0000000000000 \mathrm{E}+001.000 \mathrm{E}+01$

$29 \quad 2.0650000572205 \mathrm{E}+00 \quad 0.0000000000000 \mathrm{E}+00 \quad 0.0000000000000 \mathrm{E}+00 \quad 1.000 \mathrm{E}+01$

$302.0650000572205 \mathrm{E}+00 \quad 0.0000000000000 \mathrm{E}+00 \quad 1.0000000000000 \mathrm{E}+00 \quad 1.000 \mathrm{E}+01$

$312.0650000572205 \mathrm{E}+00 \quad 1.0000000000000 \mathrm{E}+00 \quad 0.0000000000000 \mathrm{E}+00 \quad 1.000 \mathrm{E}+01$

$32 \quad 2.0650000572205 \mathrm{E}+00 \quad 1.0000000000000 \mathrm{E}+00 \quad 1.0000000000000 \mathrm{E}+00 \quad 1.000 \mathrm{E}+01$

$332.3600001335144 \mathrm{E}+00 \quad 0.0000000000000 \mathrm{E}+00 \quad 0.0000000000000 \mathrm{E}+00 \quad 1.000 \mathrm{E}+01$

$342.3600001335144 \mathrm{E}+00 \quad 0.0000000000000 \mathrm{E}+00 \quad 1.0000000000000 \mathrm{E}+001.000 \mathrm{E}+01$

$352.3600001335144 \mathrm{E}+00 \quad 1.0000000000000 \mathrm{E}+00 \quad 0.0000000000000 \mathrm{E}+00 \quad 1.000 \mathrm{E}+01$

$362.3600001335144 \mathrm{E}+00 \quad 1.0000000000000 \mathrm{E}+00 \quad 1.0000000000000 \mathrm{E}+00 \quad 1.000 \mathrm{E}+01$

$37 \quad 2.6550002098083 \mathrm{E}+00 \quad 0.0000000000000 \mathrm{E}+00 \quad 0.0000000000000 \mathrm{E}+00 \quad 1.000 \mathrm{E}+01$

$38 \quad 2.6550002098083 \mathrm{E}+00 \quad 0.0000000000000 \mathrm{E}+00 \quad 1.0000000000000 \mathrm{E}+00 \quad 1.000 \mathrm{E}+01$

$392.6550002098083 \mathrm{E}+00 \quad 1.0000000000000 \mathrm{E}+00 \quad 0.0000000000000 \mathrm{E}+00 \quad 1.000 \mathrm{E}+01$

$40 \quad 2.6550002098083 \mathrm{E}+00 \quad 1.0000000000000 \mathrm{E}+00 \quad 1.0000000000000 \mathrm{E}+001.000 \mathrm{E}+01$

$412.9500000476837 \mathrm{E}+00 \quad 0.0000000000000 \mathrm{E}+00 \quad 0.0000000000000 \mathrm{E}+00 \quad 1.000 \mathrm{E}+01$

$42 \quad 2.9500000476837 \mathrm{E}+00 \quad 0.0000000000000 \mathrm{E}+00 \quad 1.0000000000000 \mathrm{E}+00 \quad 1.000 \mathrm{E}+01$

$43 \quad 2.9500000476837 \mathrm{E}+00 \quad 1.0000000000000 \mathrm{E}+00 \quad 0.0000000000000 \mathrm{E}+00 \quad 1.000 \mathrm{E}+01$

$442.9500000476837 \mathrm{E}+001.0000000000000 \mathrm{E}+00 \quad 1.0000000000000 \mathrm{E}+001.000 \mathrm{E}+01$

$453.0499999523163 \mathrm{E}+00 \quad 0.0000000000000 \mathrm{E}+00 \quad 0.0000000000000 \mathrm{E}+00 \quad 1.000 \mathrm{E}+01$ 
$3.0499999523163 \mathrm{E}+00 \quad 0.0000000000000 \mathrm{E}+00 \quad 1.0000000000000 \mathrm{E}+00 \quad 1.000 \mathrm{E}+01$ $3.0499999523163 \mathrm{E}+00 \quad 1.0000000000000 \mathrm{E}+00 \quad 0.0000000000000 \mathrm{E}+00 \quad 1.000 \mathrm{E}+01$ $3.0499999523163 \mathrm{E}+00 \quad 1.0000000000000 \mathrm{E}+00 \quad 1.0000000000000 \mathrm{E}+00 \quad 1.000 \mathrm{E}+01$ $\begin{array}{lllll}3.4157893657684 \mathrm{E}+00 & 0.0000000000000 \mathrm{E}+00 & 0.0000000000000 \mathrm{E}+00 & 1.000 \mathrm{E}+01\end{array}$ $3.4157893657684 \mathrm{E}+00 \quad 0.0000000000000 \mathrm{E}+00 \quad 1.0000000000000 \mathrm{E}+00 \quad 1.000 \mathrm{E}+01$ $3.4157893657684 \mathrm{E}+00 \quad 1.0000000000000 \mathrm{E}+00 \quad 0.0000000000000 \mathrm{E}+00 \quad 1.000 \mathrm{E}+01$ $3.4157893657684 \mathrm{E}+00 \quad 1.0000000000000 \mathrm{E}+00 \quad 1.0000000000000 \mathrm{E}+00 \quad 1.000 \mathrm{E}+01$ $\begin{array}{llll}3.7815790176392 \mathrm{E}+00 & 0.0000000000000 \mathrm{E}+00 & 0.0000000000000 \mathrm{E}+00 \quad 1.000 \mathrm{E}+01\end{array}$ $3.7815790176392 \mathrm{E}+00 \quad 0.0000000000000 \mathrm{E}+00 \quad 1.0000000000000 \mathrm{E}+00 \quad 1.000 \mathrm{E}+01$ $3.7815790176392 \mathrm{E}+00 \quad 1.0000000000000 \mathrm{E}+00 \quad 0.0000000000000 \mathrm{E}+00 \quad 1.000 \mathrm{E}+01$ $3.7815790176392 \mathrm{E}+001.0000000000000 \mathrm{E}+00 \quad 1.0000000000000 \mathrm{E}+00 \quad 1.000 \mathrm{E}+01$ $\begin{array}{llll}4.1473684310913 \mathrm{E}+00 & 0.0000000000000 \mathrm{E}+00 & 0.0000000000000 \mathrm{E}+00 \quad 1.000 \mathrm{E}+01\end{array}$ $4.1473684310913 \mathrm{E}+00 \quad 0.0000000000000 \mathrm{E}+00 \quad 1.0000000000000 \mathrm{E}+00 \quad 1.000 \mathrm{E}+01$ $4.1473684310913 \mathrm{E}+00 \quad 1.0000000000000 \mathrm{E}+00 \quad 0.0000000000000 \mathrm{E}+00 \quad 1.000 \mathrm{E}+01$ $4.1473684310913 \mathrm{E}+001.0000000000000 \mathrm{E}+00 \quad 1.0000000000000 \mathrm{E}+00 \quad 1.000 \mathrm{E}+01$ $\begin{array}{llll}4.5131578445435 \mathrm{E}+00 & 0.0000000000000 \mathrm{E}+00 & 0.0000000000000 \mathrm{E}+00 & 1.000 \mathrm{E}+01\end{array}$ $\begin{array}{llll}4.5131578445435 \mathrm{E}+00 & 0.0000000000000 \mathrm{E}+00 & 1.0000000000000 \mathrm{E}+00 & 1.000 \mathrm{E}+01\end{array}$ $4.5131578445435 \mathrm{E}+00 \quad 1.0000000000000 \mathrm{E}+00 \quad 0.0000000000000 \mathrm{E}+00 \quad 1.000 \mathrm{E}+01$ $\begin{array}{llll}4.5131578445435 \mathrm{E}+00 & 1.0000000000000 \mathrm{E}+00 & 1.0000000000000 \mathrm{E}+00 & 1.000 \mathrm{E}+01\end{array}$ $\begin{array}{llll}4.8789472579956 \mathrm{E}+00 & 0.0000000000000 \mathrm{E}+00 & 0.0000000000000 \mathrm{E}+00 & 1.000 \mathrm{E}+01\end{array}$ $\begin{array}{llll}4.8789472579956 \mathrm{E}+00 & 0.0000000000000 \mathrm{E}+00 & 1.0000000000000 \mathrm{E}+00 & 1.000 \mathrm{E}+01\end{array}$ $\begin{array}{llll}4.8789472579956 \mathrm{E}+00 & 1.0000000000000 \mathrm{E}+00 & 0.0000000000000 \mathrm{E}+00 & 1.000 \mathrm{E}+01\end{array}$ $4.8789472579956 \mathrm{E}+001.0000000000000 \mathrm{E}+00 \quad 1.0000000000000 \mathrm{E}+00 \quad 1.000 \mathrm{E}+01$ $\begin{array}{lllll}5.2447366714478 \mathrm{E}+00 & 0.0000000000000 \mathrm{E}+00 & 0.0000000000000 \mathrm{E}+00 & 1.000 \mathrm{E}+01\end{array}$ $5.2447366714478 \mathrm{E}+00 \quad 0.0000000000000 \mathrm{E}+00 \quad 1.0000000000000 \mathrm{E}+00 \quad 1.000 \mathrm{E}+01$ $5.2447366714478 \mathrm{E}+00 \quad 1.0000000000000 \mathrm{E}+00 \quad 0.0000000000000 \mathrm{E}+00 \quad 1.000 \mathrm{E}+01$ $5.2447366714478 \mathrm{E}+00 \quad 1.0000000000000 \mathrm{E}+00 \quad 1.0000000000000 \mathrm{E}+00 \quad 1.000 \mathrm{E}+01$ $\begin{array}{llll}5.6105260848999 \mathrm{E}+00 & 0.0000000000000 \mathrm{E}+00 & 0.0000000000000 \mathrm{E}+00 & 1.000 \mathrm{E}+01\end{array}$ $5.6105260848999 \mathrm{E}+00 \quad 0.0000000000000 \mathrm{E}+00 \quad 1.0000000000000 \mathrm{E}+00 \quad 1.000 \mathrm{E}+01$ $5.6105260848999 \mathrm{E}+00 \quad 1.0000000000000 \mathrm{E}+00 \quad 0.0000000000000 \mathrm{E}+00 \quad 1.000 \mathrm{E}+01$ $5.6105260848999 \mathrm{E}+00 \quad 1.0000000000000 \mathrm{E}+00 \quad 1.0000000000000 \mathrm{E}+00 \quad 1.000 \mathrm{E}+01$ $\begin{array}{llll}5.9763159751892 \mathrm{E}+00 & 0.0000000000000 \mathrm{E}+00 & 0.0000000000000 \mathrm{E}+00 & 1.000 \mathrm{E}+01\end{array}$ $\begin{array}{llll}5.9763159751892 \mathrm{E}+00 & 0.0000000000000 \mathrm{E}+00 & 1.0000000000000 \mathrm{E}+00 & 1.000 \mathrm{E}+01\end{array}$ $\begin{array}{llll}5.9763159751892 \mathrm{E}+00 & 1.0000000000000 \mathrm{E}+00 & 0.0000000000000 \mathrm{E}+00 & 1.000 \mathrm{E}+01\end{array}$ $\begin{array}{llll}5.9763159751892 \mathrm{E}+00 & 1.0000000000000 \mathrm{E}+00 & 1.0000000000000 \mathrm{E}+00 \quad 1.000 \mathrm{E}+01\end{array}$ $\begin{array}{lllll}6.3421053886414 \mathrm{E}+00 & 0.0000000000000 \mathrm{E}+00 & 0.0000000000000 \mathrm{E}+00 & 1.000 \mathrm{E}+01\end{array}$ $\begin{array}{llll}6.3421053886414 \mathrm{E}+00 & 0.0000000000000 \mathrm{E}+00 & 1.0000000000000 \mathrm{E}+00 & 1.000 \mathrm{E}+01\end{array}$ $6.3421053886414 \mathrm{E}+00 \quad 1.0000000000000 \mathrm{E}+00 \quad 0.0000000000000 \mathrm{E}+00 \quad 1.000 \mathrm{E}+01$ $6.3421053886414 \mathrm{E}+00 \quad 1.0000000000000 \mathrm{E}+00 \quad 1.0000000000000 \mathrm{E}+00 \quad 1.000 \mathrm{E}+01$ $\begin{array}{llll}6.7078948020935 \mathrm{E}+00 & 0.0000000000000 \mathrm{E}+00 & 0.0000000000000 \mathrm{E}+00 \quad 1.000 \mathrm{E}+01\end{array}$ $\begin{array}{llll}6.7078948020935 \mathrm{E}+00 & 0.0000000000000 \mathrm{E}+00 & 1.0000000000000 \mathrm{E}+00 & 1.000 \mathrm{E}+01\end{array}$ $6.7078948020935 \mathrm{E}+00 \quad 1.0000000000000 \mathrm{E}+00 \quad 0.0000000000000 \mathrm{E}+00 \quad 1.000 \mathrm{E}+01$ $6.7078948020935 \mathrm{E}+00 \quad 1.0000000000000 \mathrm{E}+00 \quad 1.0000000000000 \mathrm{E}+00 \quad 1.000 \mathrm{E}+01$ $\begin{array}{llll}7.0736837387085 \mathrm{E}+00 & 0.0000000000000 \mathrm{E}+00 & 0.0000000000000 \mathrm{E}+00 & 1.000 \mathrm{E}+01\end{array}$ $\begin{array}{llll}7.0736837387085 \mathrm{E}+00 & 0.0000000000000 \mathrm{E}+00 & 1.0000000000000 \mathrm{E}+00 \quad 1.000 \mathrm{E}+01\end{array}$ $\begin{array}{llll}7.0736837387085 \mathrm{E}+00 & 1.0000000000000 \mathrm{E}+00 & 0.0000000000000 \mathrm{E}+00 & 1.000 \mathrm{E}+01\end{array}$ $7.0736837387085 \mathrm{E}+00 \quad 1.0000000000000 \mathrm{E}+00 \quad 1.0000000000000 \mathrm{E}+00 \quad 1.000 \mathrm{E}+01$ $\begin{array}{llll}7.4394731521606 \mathrm{E}+00 & 0.0000000000000 \mathrm{E}+00 & 0.0000000000000 \mathrm{E}+00 & 1.000 \mathrm{E}+01\end{array}$ $7.4394731521606 \mathrm{E}+00 \quad 0.0000000000000 \mathrm{E}+00 \quad 1.0000000000000 \mathrm{E}+00 \quad 1.000 \mathrm{E}+01$ $\begin{array}{llll}7.4394731521606 \mathrm{E}+00 & 1.0000000000000 \mathrm{E}+00 & 0.0000000000000 \mathrm{E}+00 & 1.000 \mathrm{E}+01\end{array}$ $\begin{array}{llll}7.4394731521606 \mathrm{E}+00 & 1.0000000000000 \mathrm{E}+00 & 1.0000000000000 \mathrm{E}+00 & 1.000 \mathrm{E}+01\end{array}$ $\begin{array}{llll}7.8052625656128 \mathrm{E}+00 & 0.0000000000000 \mathrm{E}+00 & 0.0000000000000 \mathrm{E}+00 & 1.000 \mathrm{E}+01\end{array}$ $\begin{array}{llll}7.8052625656128 \mathrm{E}+00 & 0.0000000000000 \mathrm{E}+00 & 1.0000000000000 \mathrm{E}+00 & 1.000 \mathrm{E}+01\end{array}$ $\begin{array}{llll}7.8052625656128 \mathrm{E}+00 & 1.0000000000000 \mathrm{E}+00 & 0.0000000000000 \mathrm{E}+00 & 1.000 \mathrm{E}+01\end{array}$ $7.8052625656128 \mathrm{E}+00 \quad 1.0000000000000 \mathrm{E}+00 \quad 1.0000000000000 \mathrm{E}+00 \quad 1.000 \mathrm{E}+01$ $8.1710519790649 \mathrm{E}+00 \quad 0.0000000000000 \mathrm{E}+00 \quad 0.0000000000000 \mathrm{E}+00 \quad 1.000 \mathrm{E}+01$ $8.1710519790649 \mathrm{E}+00 \quad 0.0000000000000 \mathrm{E}+00 \quad 1.0000000000000 \mathrm{E}+00 \quad 1.000 \mathrm{E}+01$ $8.1710519790649 \mathrm{E}+00 \quad 1.0000000000000 \mathrm{E}+00 \quad 0.0000000000000 \mathrm{E}+00 \quad 1.000 \mathrm{E}+01$ $8.1710519790649 \mathrm{E}+00 \quad 1.0000000000000 \mathrm{E}+00 \quad 1.0000000000000 \mathrm{E}+00 \quad 1.000 \mathrm{E}+01$ $\begin{array}{lllll}8.5368413925171 \mathrm{E}+00 & 0.0000000000000 \mathrm{E}+00 & 0.0000000000000 \mathrm{E}+00 & 1.000 \mathrm{E}+01\end{array}$ $8.5368413925171 \mathrm{E}+00 \quad 0.0000000000000 \mathrm{E}+00 \quad 1.0000000000000 \mathrm{E}+00 \quad 1.000 \mathrm{E}+01$ $8.5368413925171 \mathrm{E}+00 \quad 1.0000000000000 \mathrm{E}+00 \quad 0.0000000000000 \mathrm{E}+00 \quad 1.000 \mathrm{E}+01$ $8.5368413925171 \mathrm{E}+00 \quad 1.0000000000000 \mathrm{E}+00 \quad 1.0000000000000 \mathrm{E}+00 \quad 1.000 \mathrm{E}+01$

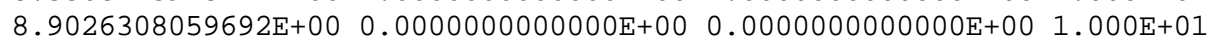




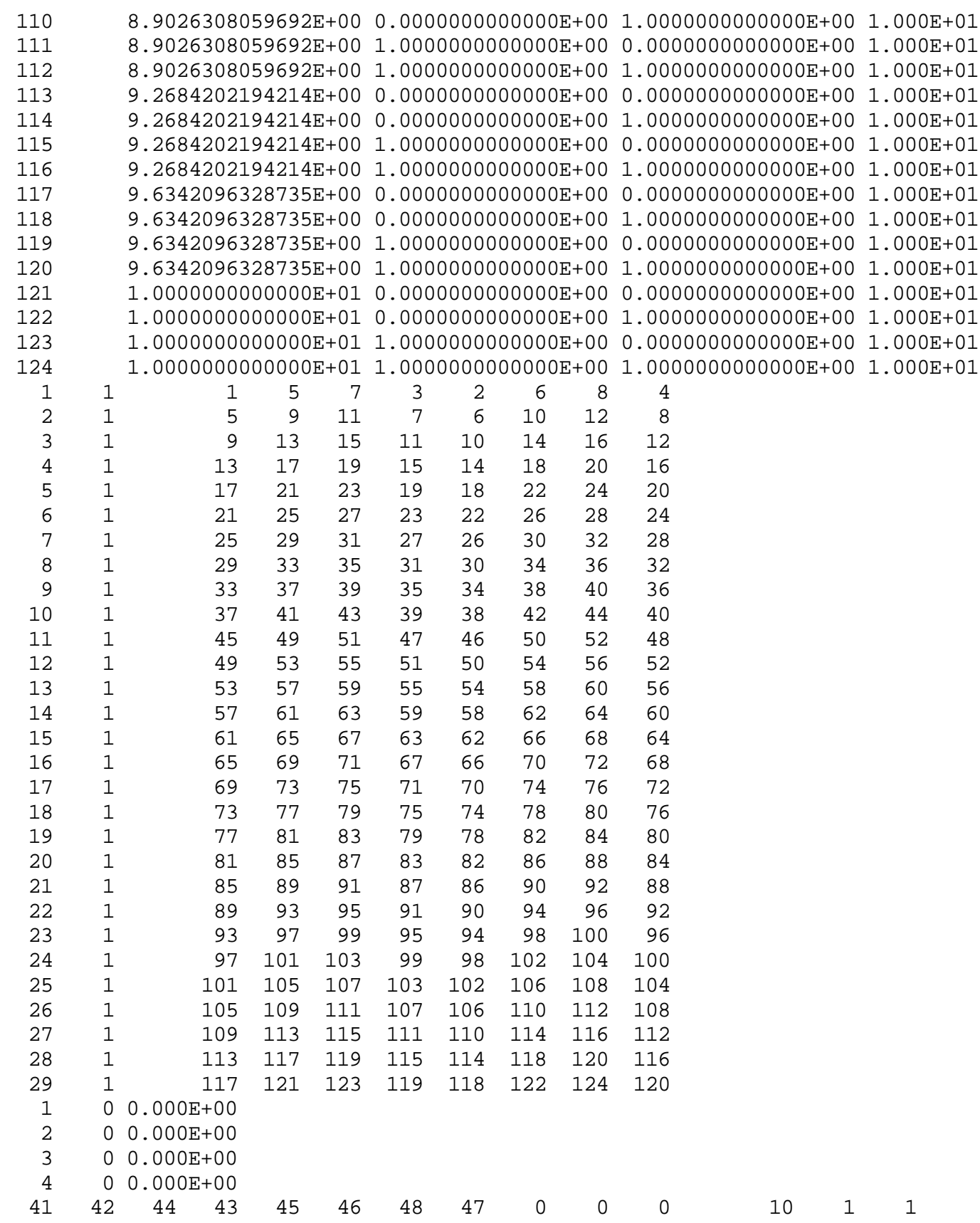

\subsection{Temporal Temperature Boundary Condition}

Problem:

This case models the transient flow of heat in a slab with a timedependent temperature boundary condition set at $\mathrm{x}=\mathrm{L}$ and a global zero-initial-temperature condition. The solutions given are for the temperature condition $T_{L}=\beta e^{v t}$ and for the $\mathrm{x}$-location of $\mathrm{x}=0.5$ at various times up to 2 seconds. 
Analytic solution:

$$
T(x, t)=\beta e^{v t} \frac{\cosh \left(x \sqrt{\frac{v}{\alpha}}\right)}{\cosh \left(L \sqrt{\frac{v}{\alpha}}\right)}-\frac{4 \beta}{\pi} \sum_{n=0}^{\infty} \frac{(-1)^{n} e^{-\alpha(2 n+1)^{2} \pi^{2} t / 4 L^{2}}}{(2 n+1)\left[1+\frac{4 v L^{2}}{(2 n+1)^{2} \pi^{2} \alpha}\right]} \cos \left[\frac{(2 n+1) \pi x}{2 L}\right]
$$

(Carslaw \& Jaeger, p.105)

Case parameters: $\quad$ Slab length - $\quad L=1$

Boundary conditions $-T_{i}=0$

Other parameters - $\quad x=0.5$

$v=0.1$

$\beta=1.0$

$\underline{\text { Results: }} \quad \underline{\text { time }} \quad \underline{T \text { (analytical) }} \underline{T(T O P A Z 3 D)}$

$\begin{array}{lll}0.10 & 0.26551 & 0.26622 \\ 0.20 & 0.45165 & 0.45206 \\ 0.25 & 0.52025 & 0.52053 \\ 0.40 & 0.68074 & 0.68074 \\ 0.50 & 0.76137 & 0.76126 \\ 0.75 & 0.90303 & 0.90280 \\ 0.80 & 0.92402 & 0.92379 \\ 1.00 & 0.99193 & 0.99162 \\ 1.50 & 1.09855 & 1.09862 \\ 2.00 & 1.17112 & 1.17115\end{array}$

Note: The analytic solutions are taken from the TOPAZ2D verification manual.

Input file:

C TOPAZ3D input file for transient linear temperature boundary condition ( $t$ )

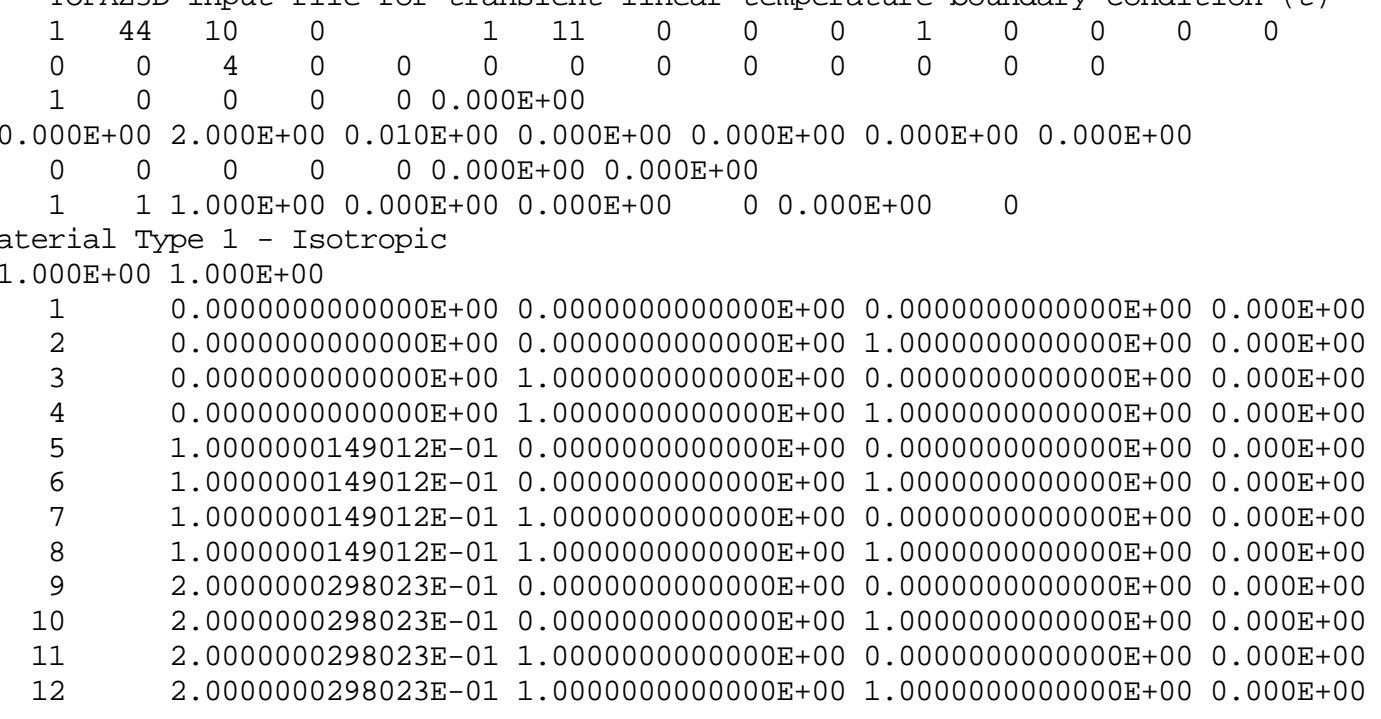




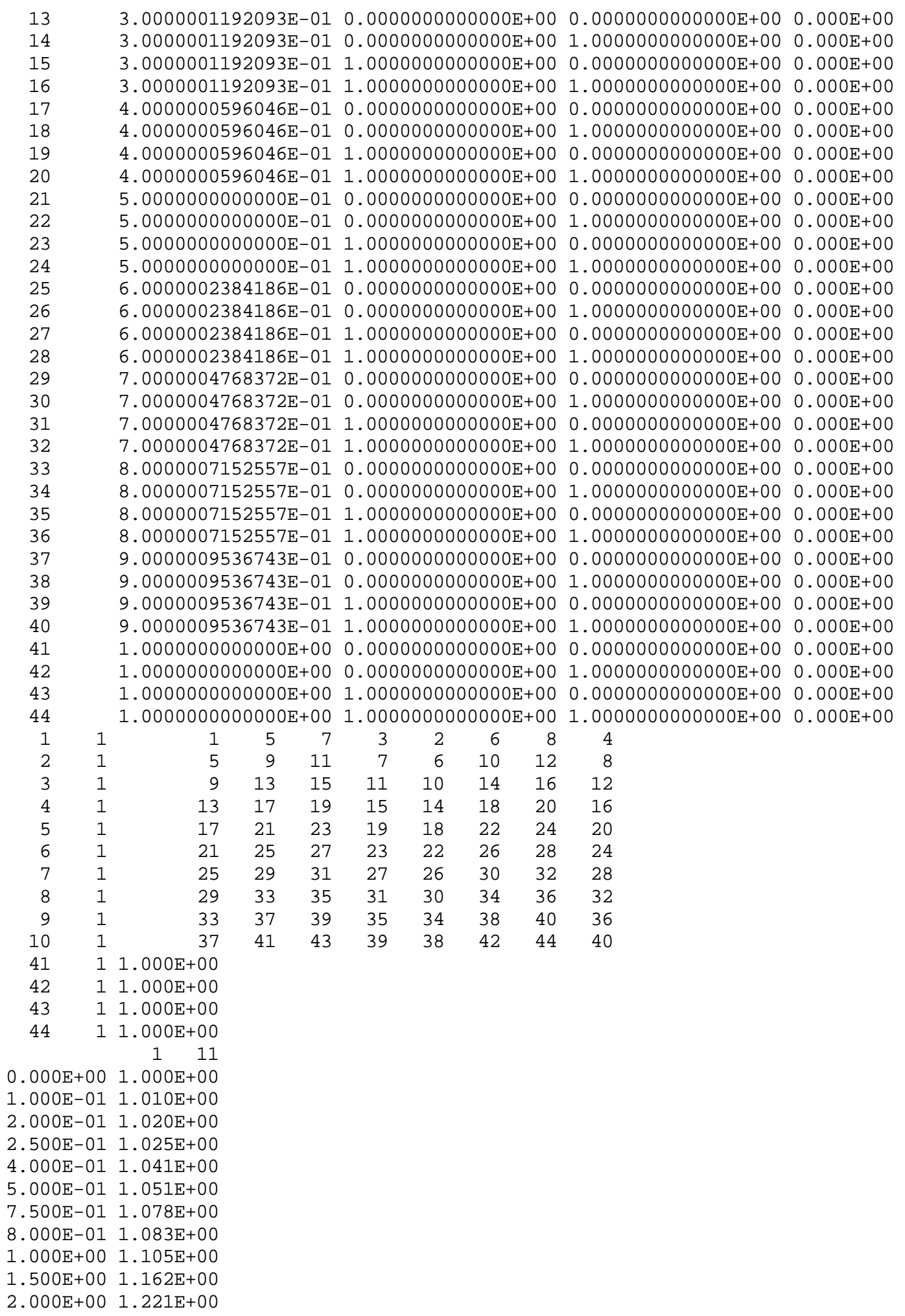


Problem: $\quad$ This case models the transient flow of heat in a slab with a timedependent flux boundary condition set at $\mathrm{x}=\mathrm{L}$ and a global zeroinitial-temperature condition. The solutions given are for the flux condition $q^{\prime \prime}=q_{i} t^{\frac{1}{2} m}$ and for the $\mathrm{x}$-location of $\mathrm{x}=0.5$ at various times up to 2 seconds.

Analytic solution:

$$
T(x, t)=\frac{2^{m+1} q_{i} \sqrt{\alpha t} t^{(m+1)} \Gamma\left(\frac{1}{2} m+1\right)}{k} \sum_{n=0}^{\infty}\left[i^{m+1} \operatorname{erfc} \frac{(2 n+1) L-x}{2 \sqrt{\alpha t}}+i^{m+1} \operatorname{erfc} \frac{(2 n+1) L+x}{2 \sqrt{\alpha t}}\right]
$$

(Carslaw \& Jaeger, p.113)

For the case in which $m=-1$, the solution is

$T(x, t)=\frac{q_{i}}{L} \sqrt{\frac{\pi \alpha}{k}} \sum_{n=0}^{\infty}\left[\operatorname{erfc} \frac{(2 n+1) L-x}{2 \sqrt{\alpha t}}+\operatorname{erfc} \frac{(2 n+1) L+x}{2 \sqrt{\alpha t}}\right] \quad$ (Drayton, p.30)

Case parameters: $\quad$ Slab length - $\quad L=1$

Boundary conditions $-T_{i}=0$

Other parameters - $\quad x=0.5$

$\alpha=1.0$

$k=1.0$

$m=-1.0$

Results: $\quad \underline{\text { time }} \quad \underline{T \text { (analytical) }} \underline{T \text { (TOPAZ3D) }}$

$\begin{array}{lll}0.10 & 0.46855 & 0.42692 \\ 0.20 & 0.79225 & 0.80369 \\ 0.25 & 0.91069 & 0.92589 \\ 0.40 & 1.19634 & 1.21587 \\ 0.50 & 1.35341 & 1.37414 \\ 0.75 & 1.68295 & 1.70712 \\ 0.80 & 1.74138 & 1.76584 \\ 1.00 & 1.95770 & 1.98291 \\ 1.50 & 2.41513 & 2.44512 \\ 2.00 & 2.79875 & 2.83086\end{array}$

Note: The analytical solutions are taken from the TOPAZ2D verification manual. 


\section{Input file:}

c TOPAZ3D input file for transient linear flux boundary condition (t)

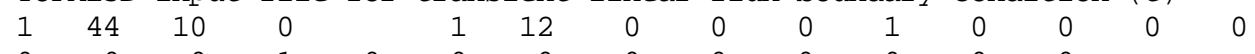

$\begin{array}{cccccc}0 & 0 & 0 & 1 & 0 & 0 \\ 1 & 0 & 0 & 0 & 0 & 0.000 \mathrm{E}+0\end{array}$

$\begin{array}{llllllll}0.000 \mathrm{E}+00 & 2.000 \mathrm{E}+00 & 1.000 \mathrm{E}-02 & 0.000 \mathrm{E}+00 & 0.000 \mathrm{E}+00 & 0.000 \mathrm{E}+00 & 0.000 \mathrm{E}+00\end{array}$

$\begin{array}{lllllll}0 & 0 & 0 & 0 & 0 & 0.000 \mathrm{E}+00 & 0.000 \mathrm{E}+00\end{array}$

$\begin{array}{llllllll}1 & 1 & 1.000 \mathrm{E}+00 & 0.000 \mathrm{E}+00 & 0.000 \mathrm{E}+00 & 0 & 0.000 \mathrm{E}+00 & 0\end{array}$

Material Type 1 - Isotropic

$1.000 \mathrm{E}+001.000 \mathrm{E}+00$

$10.0000000000000 \mathrm{E}+00 \quad 0.0000000000000 \mathrm{E}+00 \quad 0.0000000000000 \mathrm{E}+00 \quad 0.000 \mathrm{E}+00$

$2 \quad 0.0000000000000 \mathrm{E}+00 \quad 0.0000000000000 \mathrm{E}+00 \quad 1.0000000000000 \mathrm{E}+00 \quad 0.000 \mathrm{E}+00$

$3 \quad 0.0000000000000 \mathrm{E}+001.0000000000000 \mathrm{E}+00 \quad 0.0000000000000 \mathrm{E}+00 \quad 0.000 \mathrm{E}+00$

$4 \quad 0.0000000000000 \mathrm{E}+001.0000000000000 \mathrm{E}+00 \quad 1.0000000000000 \mathrm{E}+00 \quad 0.000 \mathrm{E}+00$

$5 \quad 1.0000000149012 \mathrm{E}-01 \quad 0.0000000000000 \mathrm{E}+00 \quad 0.0000000000000 \mathrm{E}+00 \quad 0.000 \mathrm{E}+00$

$6 \quad 1.0000000149012 \mathrm{E}-010.0000000000000 \mathrm{E}+00 \quad 1.0000000000000 \mathrm{E}+00 \quad 0.000 \mathrm{E}+00$

$7 \quad 1.0000000149012 \mathrm{E}-01 \quad 1.0000000000000 \mathrm{E}+00 \quad 0.0000000000000 \mathrm{E}+00 \quad 0.000 \mathrm{E}+00$

$8 \quad 1.0000000149012 \mathrm{E}-011.0000000000000 \mathrm{E}+001.0000000000000 \mathrm{E}+00 \quad 0.000 \mathrm{E}+00$

$92.0000000298023 \mathrm{E}-01 \quad 0.0000000000000 \mathrm{E}+00 \quad 0.0000000000000 \mathrm{E}+00 \quad 0.000 \mathrm{E}+00$

$10 \quad 2.0000000298023 \mathrm{E}-01 \quad 0.0000000000000 \mathrm{E}+00 \quad 1.0000000000000 \mathrm{E}+00 \quad 0.000 \mathrm{E}+00$

$2.0000000298023 \mathrm{E}-01 \quad 1.0000000000000 \mathrm{E}+00 \quad 0.0000000000000 \mathrm{E}+00 \quad 0.000 \mathrm{E}+00$

$2.0000000298023 \mathrm{E}-01 \quad 1.0000000000000 \mathrm{E}+00 \quad 1.0000000000000 \mathrm{E}+00 \quad 0.000 \mathrm{E}+00$

$3.0000001192093 \mathrm{E}-01 \quad 0.0000000000000 \mathrm{E}+00 \quad 0.0000000000000 \mathrm{E}+00 \quad 0.000 \mathrm{E}+00$

$3.0000001192093 \mathrm{E}-01 \quad 0.0000000000000 \mathrm{E}+00 \quad 1.0000000000000 \mathrm{E}+00 \quad 0.000 \mathrm{E}+00$

$3.0000001192093 \mathrm{E}-01 \quad 1.0000000000000 \mathrm{E}+00 \quad 0.0000000000000 \mathrm{E}+00 \quad 0.000 \mathrm{E}+00$

$3.0000001192093 \mathrm{E}-01 \quad 1.0000000000000 \mathrm{E}+00 \quad 1.0000000000000 \mathrm{E}+00 \quad 0.000 \mathrm{E}+00$

$\begin{array}{llll}4.0000000596046 \mathrm{E}-01 & 0.0000000000000 \mathrm{E}+00 & 0.0000000000000 \mathrm{E}+00 & 0.000 \mathrm{E}+00\end{array}$

$4.0000000596046 \mathrm{E}-01 \quad 0.0000000000000 \mathrm{E}+00 \quad 1.0000000000000 \mathrm{E}+00 \quad 0.000 \mathrm{E}+00$

$4.0000000596046 \mathrm{E}-01 \quad 1.0000000000000 \mathrm{E}+00 \quad 0.0000000000000 \mathrm{E}+00 \quad 0.000 \mathrm{E}+00$

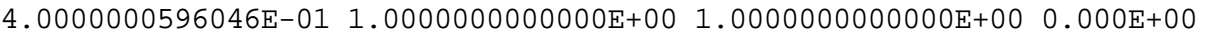

$5.0000000000000 \mathrm{E}-01 \quad 0.0000000000000 \mathrm{E}+00 \quad 0.0000000000000 \mathrm{E}+00 \quad 0.000 \mathrm{E}+00$

$5.0000000000000 \mathrm{E}-01 \quad 0.0000000000000 \mathrm{E}+00 \quad 1.0000000000000 \mathrm{E}+00 \quad 0.000 \mathrm{E}+00$

$5.0000000000000 \mathrm{E}-01 \quad 1.0000000000000 \mathrm{E}+00 \quad 0.0000000000000 \mathrm{E}+00 \quad 0.000 \mathrm{E}+00$

$5.0000000000000 \mathrm{E}-01 \quad 1.0000000000000 \mathrm{E}+00 \quad 1.0000000000000 \mathrm{E}+00 \quad 0.000 \mathrm{E}+00$

$\begin{array}{llll}6.0000002384186 \mathrm{E}-01 & 0.0000000000000 \mathrm{E}+00 & 0.0000000000000 \mathrm{E}+00 & 0.000 \mathrm{E}+00\end{array}$

$\begin{array}{lll}6.0000002384186 \mathrm{E}-01 & 0.0000000000000 \mathrm{E}+00 & 1.0000000000000 \mathrm{E}+00 \quad 0.000 \mathrm{E}+00\end{array}$

$6.0000002384186 \mathrm{E}-01 \quad 1.0000000000000 \mathrm{E}+00 \quad 0.0000000000000 \mathrm{E}+00 \quad 0.000 \mathrm{E}+00$

$6.0000002384186 \mathrm{E}-01 \quad 1.0000000000000 \mathrm{E}+00 \quad 1.0000000000000 \mathrm{E}+00 \quad 0.000 \mathrm{E}+00$

$7.0000004768372 \mathrm{E}-01 \quad 0.0000000000000 \mathrm{E}+00 \quad 0.0000000000000 \mathrm{E}+00 \quad 0.000 \mathrm{E}+00$

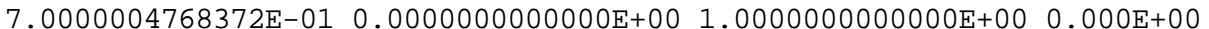

$7.0000004768372 \mathrm{E}-01 \quad 1.0000000000000 \mathrm{E}+00 \quad 0.0000000000000 \mathrm{E}+00 \quad 0.000 \mathrm{E}+00$

$7.0000004768372 \mathrm{E}-01 \quad 1.0000000000000 \mathrm{E}+00 \quad 1.0000000000000 \mathrm{E}+00 \quad 0.000 \mathrm{E}+00$

$8.0000007152557 \mathrm{E}-01 \quad 0.0000000000000 \mathrm{E}+00 \quad 0.0000000000000 \mathrm{E}+00 \quad 0.000 \mathrm{E}+00$

$8.0000007152557 \mathrm{E}-01 \quad 0.0000000000000 \mathrm{E}+00 \quad 1.0000000000000 \mathrm{E}+00 \quad 0.000 \mathrm{E}+00$

$8.0000007152557 \mathrm{E}-01 \quad 1.0000000000000 \mathrm{E}+00 \quad 0.0000000000000 \mathrm{E}+00 \quad 0.000 \mathrm{E}+00$

$8.0000007152557 \mathrm{E}-01 \quad 1.0000000000000 \mathrm{E}+00 \quad 1.0000000000000 \mathrm{E}+00 \quad 0.000 \mathrm{E}+00$

$9.0000009536743 \mathrm{E}-01 \quad 0.0000000000000 \mathrm{E}+00 \quad 0.0000000000000 \mathrm{E}+00 \quad 0.000 \mathrm{E}+00$

$9.0000009536743 \mathrm{E}-01 \quad 0.0000000000000 \mathrm{E}+00 \quad 1.0000000000000 \mathrm{E}+00 \quad 0.000 \mathrm{E}+00$

$9.0000009536743 \mathrm{E}-01 \quad 1.0000000000000 \mathrm{E}+00 \quad 0.0000000000000 \mathrm{E}+00 \quad 0.000 \mathrm{E}+00$

$9.0000009536743 \mathrm{E}-01 \quad 1.0000000000000 \mathrm{E}+00 \quad 1.0000000000000 \mathrm{E}+00 \quad 0.000 \mathrm{E}+00$

$1.0000000000000 \mathrm{E}+00 \quad 0.0000000000000 \mathrm{E}+00 \quad 0.0000000000000 \mathrm{E}+00 \quad 0.000 \mathrm{E}+00$

$1.0000000000000 \mathrm{E}+00 \quad 0.0000000000000 \mathrm{E}+00 \quad 1.0000000000000 \mathrm{E}+00 \quad 0.000 \mathrm{E}+00$

$1.0000000000000 \mathrm{E}+001.0000000000000 \mathrm{E}+00 \quad 0.0000000000000 \mathrm{E}+00 \quad 0.000 \mathrm{E}+00$

$1.0000000000000 \mathrm{E}+001.0000000000000 \mathrm{E}+00 \quad 1.0000000000000 \mathrm{E}+00 \quad 0.000 \mathrm{E}+00$

1

1

1

$\begin{array}{rrrrrrrr}1 & 5 & 7 & 3 & 2 & 6 & 8 & 4 \\ 5 & 9 & 11 & 7 & 6 & 10 & 12 & 8 \\ 9 & 13 & 15 & 11 & 10 & 14 & 16 & 12 \\ 13 & 17 & 19 & 15 & 14 & 18 & 20 & 16 \\ 17 & 21 & 23 & 19 & 18 & 22 & 24 & 20 \\ 21 & 25 & 27 & 23 & 22 & 26 & 28 & 24 \\ 25 & 29 & 31 & 27 & 26 & 30 & 32 & 28\end{array}$




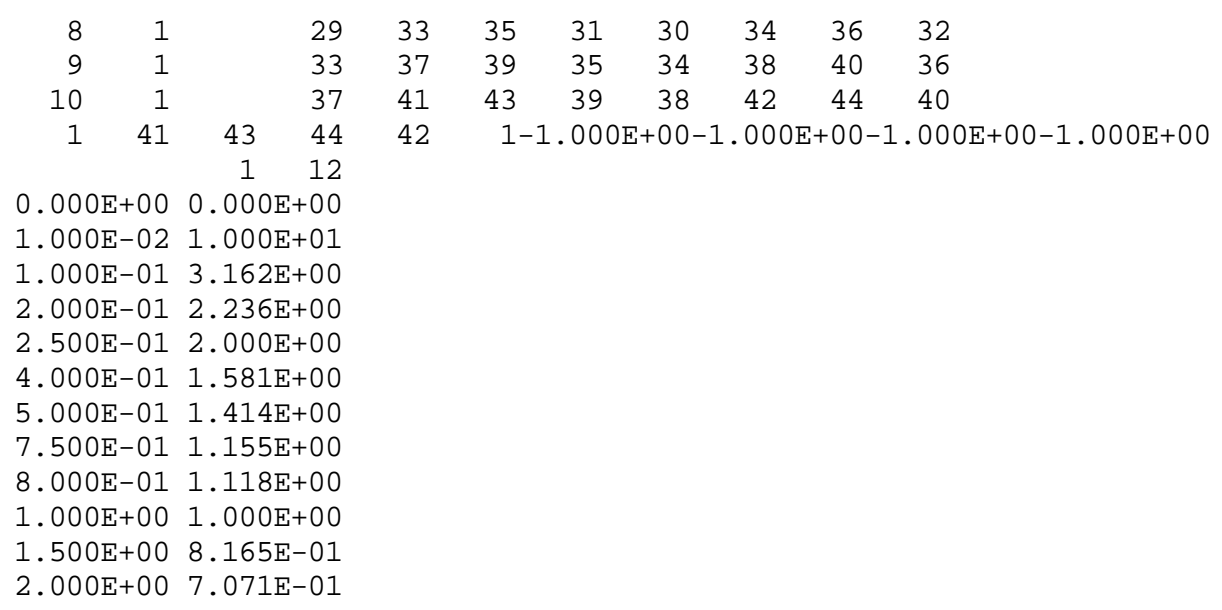

3.7

Temporal Convection Boundary Condition

Problem: $\quad$ This case models the transient flow of heat in a slab with a timedependent convection boundary condition set at $\mathrm{x}=\mathrm{L}$ and a global zero-initial-temperature condition. The solutions given are for the convection condition $T_{\infty}=C t$ and for the $\mathrm{x}$-location of $\mathrm{x}=0.5$ at various times up to 2 seconds.

Analytic solution:

$T(x, t)=C t+\frac{k\left(h x^{2}-L^{2} h-2 L\right)}{2 \alpha h}+\frac{2 h C}{\alpha} \sum_{n=1}^{\infty} e^{-\alpha \lambda_{n}^{2} t} \frac{\cos \left(\lambda_{n} x\right)}{\lambda_{n}^{2}\left[\left(h^{2}+\lambda_{n}^{2}\right) L+h\right] \cos \left(\lambda_{n} L\right)}$

where $\lambda_{\mathrm{n}}$ are the positive roots of

$$
\lambda_{n} \tan \left(\lambda_{n} L\right)=h
$$

(Carslaw \& Jaeger, p.127)

Case parameters: $\quad$ Slab length - $\quad L=1$

Boundary conditions $-T_{i}=0$

Other parameters - $\quad x=0.5$

$\alpha=1.0$

$h=1.0$

$C=1.0$

Results: $\quad \underline{\text { time }} \quad \underline{\mathrm{T} \text { (analytical) }} \underline{\mathrm{T} \text { (TOPAZ3D) }}$

$\begin{array}{lll}0.10 & 0.00174 & 0.00175 \\ 0.20 & 0.01032 & 0.01025 \\ 0.25 & 0.01718 & 0.01710\end{array}$




$\begin{array}{lll}0.40 & 0.04709 & 0.04697 \\ 0.50 & 0.07416 & 0.07402 \\ 0.75 & 0.16381 & 0.16364 \\ 0.80 & 0.18515 & 0.18497 \\ 1.00 & 0.28055 & 0.28036 \\ 1.50 & 0.57777 & 0.57757 \\ 2.00 & 0.93772 & 0.93754\end{array}$

Note: The analytical solutions are taken from the TOPAZ2D verification manual.

\section{Input file:}

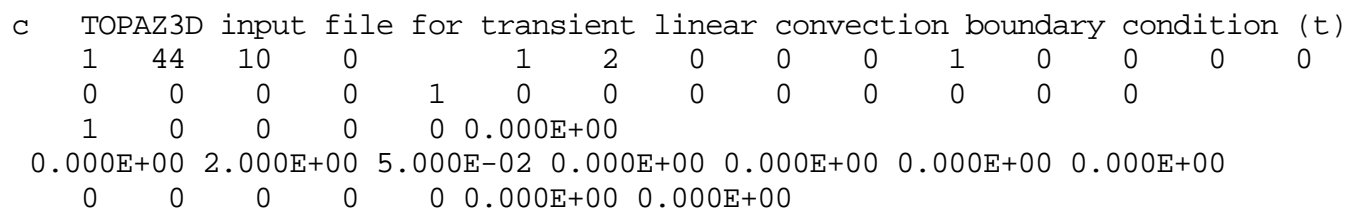


1

$11.000 \mathrm{E}+00 \quad 0.000 \mathrm{E}+00 \quad 0.000 \mathrm{E}+00$

$0.000 \mathrm{E}+00$

0

Material Type 1 - Isotropic

$1.000 \mathrm{E}+001.000 \mathrm{E}+00$

$1 \quad 0.0000000000000 \mathrm{E}+00 \quad 0.0000000000000 \mathrm{E}+00 \quad 0.0000000000000 \mathrm{E}+00 \quad 0.000 \mathrm{E}+00$

$2 \quad 0.0000000000000 \mathrm{E}+00 \quad 0.0000000000000 \mathrm{E}+00 \quad 1.0000000000000 \mathrm{E}+00 \quad 0.000 \mathrm{E}+00$

$3 \quad 0.0000000000000 \mathrm{E}+00 \quad 1.0000000000000 \mathrm{E}+00 \quad 0.0000000000000 \mathrm{E}+00 \quad 0.000 \mathrm{E}+00$

$4 \quad 0.0000000000000 \mathrm{E}+001.0000000000000 \mathrm{E}+00 \quad 1.0000000000000 \mathrm{E}+00 \quad 0.000 \mathrm{E}+00$

$5 \quad 1.0000000149012 \mathrm{E}-01 \quad 0.0000000000000 \mathrm{E}+00 \quad 0.0000000000000 \mathrm{E}+00 \quad 0.000 \mathrm{E}+00$

$6 \quad 1.0000000149012 \mathrm{E}-01 \quad 0.0000000000000 \mathrm{E}+00 \quad 1.0000000000000 \mathrm{E}+00 \quad 0.000 \mathrm{E}+00$

$7 \quad 1.0000000149012 \mathrm{E}-01 \quad 1.0000000000000 \mathrm{E}+00 \quad 0.0000000000000 \mathrm{E}+00 \quad 0.000 \mathrm{E}+00$

$8 \quad 1.0000000149012 \mathrm{E}-01 \quad 1.0000000000000 \mathrm{E}+00 \quad 1.0000000000000 \mathrm{E}+00 \quad 0.000 \mathrm{E}+00$

$92.0000000298023 \mathrm{E}-01 \quad 0.0000000000000 \mathrm{E}+00 \quad 0.0000000000000 \mathrm{E}+00 \quad 0.000 \mathrm{E}+00$

$102.0000000298023 \mathrm{E}-01 \quad 0.0000000000000 \mathrm{E}+00 \quad 1.0000000000000 \mathrm{E}+00 \quad 0.000 \mathrm{E}+00$

$112.0000000298023 \mathrm{E}-01 \quad 1.0000000000000 \mathrm{E}+00 \quad 0.0000000000000 \mathrm{E}+00 \quad 0.000 \mathrm{E}+00$

$122.0000000298023 \mathrm{E}-01 \quad 1.0000000000000 \mathrm{E}+00 \quad 1.0000000000000 \mathrm{E}+00 \quad 0.000 \mathrm{E}+00$

$133.0000001192093 \mathrm{E}-01 \quad 0.0000000000000 \mathrm{E}+00 \quad 0.0000000000000 \mathrm{E}+00 \quad 0.000 \mathrm{E}+00$

$143.0000001192093 \mathrm{E}-01 \quad 0.0000000000000 \mathrm{E}+00 \quad 1.0000000000000 \mathrm{E}+00 \quad 0.000 \mathrm{E}+00$

$153.0000001192093 \mathrm{E}-01 \quad 1.0000000000000 \mathrm{E}+00 \quad 0.0000000000000 \mathrm{E}+00 \quad 0.000 \mathrm{E}+00$

$163.0000001192093 \mathrm{E}-01 \quad 1.0000000000000 \mathrm{E}+00 \quad 1.0000000000000 \mathrm{E}+00 \quad 0.000 \mathrm{E}+00$

$174.0000000596046 \mathrm{E}-01 \quad 0.0000000000000 \mathrm{E}+00 \quad 0.0000000000000 \mathrm{E}+00 \quad 0.000 \mathrm{E}+00$

$184.0000000596046 \mathrm{E}-01 \quad 0.0000000000000 \mathrm{E}+00 \quad 1.0000000000000 \mathrm{E}+00 \quad 0.000 \mathrm{E}+00$

$194.0000000596046 \mathrm{E}-01 \quad 1.0000000000000 \mathrm{E}+00 \quad 0.0000000000000 \mathrm{E}+00 \quad 0.000 \mathrm{E}+00$

$204.0000000596046 \mathrm{E}-01 \quad 1.0000000000000 \mathrm{E}+00 \quad 1.0000000000000 \mathrm{E}+00 \quad 0.000 \mathrm{E}+00$

$215.0000000000000 \mathrm{E}-01 \quad 0.0000000000000 \mathrm{E}+00 \quad 0.0000000000000 \mathrm{E}+00 \quad 0.000 \mathrm{E}+00$

$22 \quad 5.0000000000000 \mathrm{E}-01 \quad 0.0000000000000 \mathrm{E}+00 \quad 1.0000000000000 \mathrm{E}+00 \quad 0.000 \mathrm{E}+00$

$235.0000000000000 \mathrm{E}-01 \quad 1.0000000000000 \mathrm{E}+00 \quad 0.0000000000000 \mathrm{E}+00 \quad 0.000 \mathrm{E}+00$

$245.0000000000000 \mathrm{E}-01 \quad 1.0000000000000 \mathrm{E}+001.0000000000000 \mathrm{E}+00 \quad 0.000 \mathrm{E}+00$

$256.0000002384186 \mathrm{E}-01 \quad 0.0000000000000 \mathrm{E}+00 \quad 0.0000000000000 \mathrm{E}+00 \quad 0.000 \mathrm{E}+00$

$26 \quad 6.0000002384186 \mathrm{E}-01 \quad 0.0000000000000 \mathrm{E}+00 \quad 1.0000000000000 \mathrm{E}+00 \quad 0.000 \mathrm{E}+00$

$276.0000002384186 \mathrm{E}-01 \quad 1.0000000000000 \mathrm{E}+00 \quad 0.0000000000000 \mathrm{E}+00 \quad 0.000 \mathrm{E}+00$

$286.0000002384186 \mathrm{E}-01 \quad 1.0000000000000 \mathrm{E}+00 \quad 1.0000000000000 \mathrm{E}+00 \quad 0.000 \mathrm{E}+00$

$297.0000004768372 \mathrm{E}-01 \quad 0.0000000000000 \mathrm{E}+00 \quad 0.0000000000000 \mathrm{E}+00 \quad 0.000 \mathrm{E}+00$

$307.0000004768372 \mathrm{E}-01 \quad 0.0000000000000 \mathrm{E}+00 \quad 1.0000000000000 \mathrm{E}+00 \quad 0.000 \mathrm{E}+00$

$317.0000004768372 \mathrm{E}-01 \quad 1.0000000000000 \mathrm{E}+00 \quad 0.0000000000000 \mathrm{E}+00 \quad 0.000 \mathrm{E}+00$

$327.0000004768372 \mathrm{E}-01 \quad 1.0000000000000 \mathrm{E}+00 \quad 1.0000000000000 \mathrm{E}+00 \quad 0.000 \mathrm{E}+00$

$338.0000007152557 \mathrm{E}-01 \quad 0.0000000000000 \mathrm{E}+00 \quad 0.0000000000000 \mathrm{E}+00 \quad 0.000 \mathrm{E}+00$

$348.0000007152557 \mathrm{E}-01 \quad 0.0000000000000 \mathrm{E}+00 \quad 1.0000000000000 \mathrm{E}+00 \quad 0.000 \mathrm{E}+00$

$358.0000007152557 \mathrm{E}-01 \quad 1.0000000000000 \mathrm{E}+00 \quad 0.0000000000000 \mathrm{E}+00 \quad 0.000 \mathrm{E}+00$

$368.0000007152557 \mathrm{E}-01 \quad 1.0000000000000 \mathrm{E}+00 \quad 1.0000000000000 \mathrm{E}+00 \quad 0.000 \mathrm{E}+00$

$379.0000009536743 \mathrm{E}-01 \quad 0.0000000000000 \mathrm{E}+00 \quad 0.0000000000000 \mathrm{E}+00 \quad 0.000 \mathrm{E}+00$

$389.0000009536743 \mathrm{E}-01 \quad 0.0000000000000 \mathrm{E}+00 \quad 1.0000000000000 \mathrm{E}+00 \quad 0.000 \mathrm{E}+00$

$399.0000009536743 \mathrm{E}-01 \quad 1.0000000000000 \mathrm{E}+00 \quad 0.0000000000000 \mathrm{E}+00 \quad 0.000 \mathrm{E}+00$

$40 \quad 9.0000009536743 \mathrm{E}-01 \quad 1.0000000000000 \mathrm{E}+00 \quad 1.0000000000000 \mathrm{E}+00 \quad 0.000 \mathrm{E}+00$

$411.0000000000000 \mathrm{E}+00 \quad 0.0000000000000 \mathrm{E}+00 \quad 0.0000000000000 \mathrm{E}+00 \quad 0.000 \mathrm{E}+00$

$42 \quad 1.0000000000000 \mathrm{E}+00 \quad 0.0000000000000 \mathrm{E}+00 \quad 1.0000000000000 \mathrm{E}+00 \quad 0.000 \mathrm{E}+00$

$43 \quad 1.0000000000000 \mathrm{E}+001.0000000000000 \mathrm{E}+00 \quad 0.0000000000000 \mathrm{E}+00 \quad 0.000 \mathrm{E}+00$

$441.0000000000000 \mathrm{E}+001.0000000000000 \mathrm{E}+00 \quad 1.0000000000000 \mathrm{E}+00 \quad 0.000 \mathrm{E}+00$

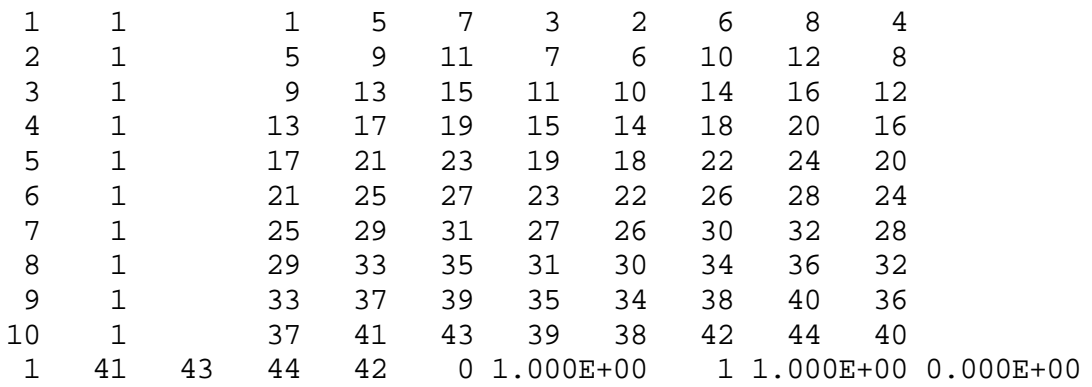

$0.000 \mathrm{E}+00 \quad 0.000 \mathrm{E}+00$

$2.000 \mathrm{E}+00 \quad 2.000 \mathrm{E}+00$ 


\subsection{Bibliography}

1. Carlsaw, H.S. and Jaeger, J.C. Conduction of Heat in Solids. Oxford University Press, 1959.

2. Drayton, Marcus. “TOPAZ2D Validation Status Report.” UCRL-ID- 106550. Lawrence Livermore National Laboratory, 1990.

3. Incropera, Frank and DeWitt, David. Fundamentals of Heat and Mass Transfer. John Wiley \& Sons, Inc., 1996. 